\title{
THE ROLE OF THE INNATE IMMUNE SYSTEM IN MICROBIALLY INDUCED INTESTINAL INFLAMMATION AND NEOPLASIA
}

\author{
A Dissertation \\ presented to \\ Faculty of the Graduate School \\ University of Missouri \\ In Partial Fulfillment \\ of the Requirements for the Degree \\ Doctor of Philosophy \\ by \\ AARON C. ERICSSON \\ Dr. Craig Franklin, Dissertation Supervisor
}

May 2013 
(C) Copyright by Aaron C. Ericsson 2013

All Rights Reserved 
The undersigned, appointed by the dean of the Graduate School, have examined the dissertation entitled

\title{
THE ROLE OF THE INNATE IMMUNE SYSTEM IN MICROBIALLY INDUCED
} INTESTINAL INFLAMMATION AND NEOPLASIA

\author{
presented by Aaron C. Ericsson,
}

a candidate for the degree of doctor of philosophy,

and hereby certify that, in their opinion, it is worthy of acceptance.

Professor Craig Franklin

Professor Habib Zaghouani

Professor David Lee

Professor Daniel Hassett

Professor Matthew Myles

Professor Michael Lewis 


\section{DEDICATION}

This work is dedicated to my wonderful family, both immediate and extended. 


\section{ACKNOWLEDGMENTS}

I would like to acknowledge the assistance provided by the following people:

First and foremost, my mentor and advisor Craig Franklin for being not just an excellent teacher and guide in the complex world of research, but also a true friend

Past and present colleagues in the Franklin laboratory, Drs. Lydia Cook and Drew Hillhouse, Giedre Turner, BJ Livingston, and Greg Purdy (honorary)

The members of my committee, Drs. Habib Zaghouani, David Lee, Dan Hassett, Matt Myles, and Michael Lewis

Past and present faculty of the Department of Veterinary Pathobiology, and in particular Drs. Elizabeth Bryda, Cindy Besch-Williford, Bob Livingston, Earl Steffen, Lela Riley, and John Critser (R.I.P.)

Past and present members of the University of Missouri Comparative Medicine Program 


\section{TABLE OF CONTENTS}

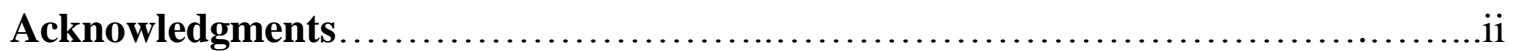

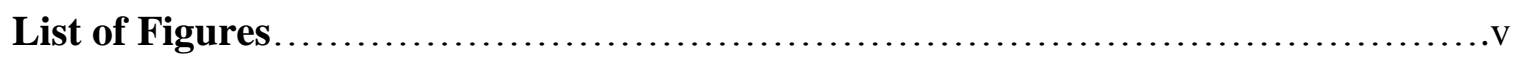

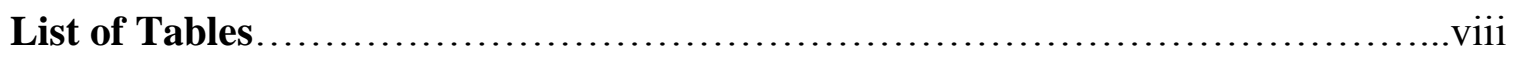

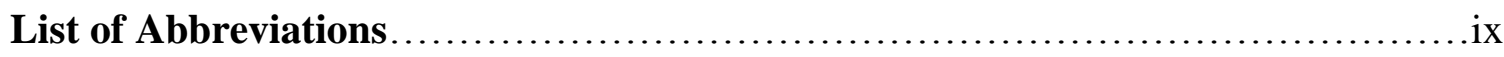

Abstract................................................................

CHAPTER 1 - Modeling of Inflammatory Bowel Disease and Colitis-associated Colorectal Cancer

Introduction.........................................................

Significance of Inflammatory Bowel Disease .............................2

Pathogenesis of Inflammatory Bowel Disease ...........................6

Animal models of Inflammatory Bowel Disease $\ldots \ldots \ldots \ldots \ldots \ldots \ldots \ldots \ldots \ldots \ldots \ldots$

Significance of Colitis-associated Colorectal Cancer..........................19

Pathogenesis of Colitis-associated Colorectal Cancer........................20

Animal Models of Colitis-associated Colorectal Cancer......................22

CHAPTER 2 - The Role of CD8 ${ }^{+}$Dendritic Cells in Helicobacter hepaticus-induced Cecal Inflammation

Overview.........................................................26

Materials and Methods................................................ 32

Results.........................................................40

Discussion............................................................... 51

CHAPTER 3 - Noninvasive Detection of Colitis-associated Colorectal Cancer in Helicobacter bilis-infected Smad3-deficient Mice

Overview......................................................... 72

Materials and Methods............................................. 75

Results............................................................... 80 
Discussion.

CHAPTER 4 - The Effect of Murine Norovirus on Two Chemically Induced Models of Inflammatory Bowel Disease

Overview.......................................................... 105

Materials and Methods................................................... 113

Results............................................................117

Discussion........................................................... 119

CHAPTER 5 - Summary and Future Direction............................. 123

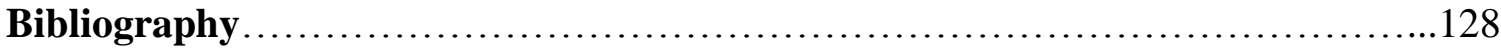

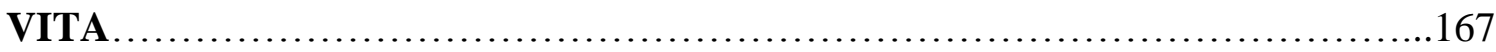




\section{LIST OF FIGURES}

Figure $\quad$ Page

Figure 1.1: Molecular pathogenesis of sporadic and colitis-associated colorectal

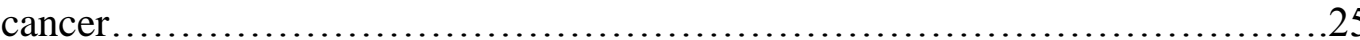

Figure 2.1: Histological appearance of cecum in H. hepaticus-infected wild type A/J, $\operatorname{Rag} 2^{-/-} \mathrm{A} / \mathrm{J}$ and wild type C57BL/6 mice .62

Figure 2.2: Total cecal lamina propria cells and cecal dendritic cells in naïve, 4d PI, and 90d PI A/J and C57BL/6 mice. 63

Figure 2.3: Flow cytometry gating strategy for dendritic cell subsets .64

Figure 2.4: Cecal dendritic cell subsets in naïve $\mathrm{A} / \mathrm{J}$ and $\mathrm{C} 57 \mathrm{BL} / 6$ mice. .65

Figure 2.5: Cecal dendritic cell subsets in 4d PI A/J and C57BL/6 mice........ .66

Figure 2.6: Cecal lamina propria cells in 90d PI A/J and C57BL/6 mice.

Figure 2.7: IL-12/23p40 production in cells from naïve A/J and C57BL/6 mice. .68

Figure 2.8: IL-12/23p40, TNF- $\alpha$, CXCL10, and CCL5 production in cells from 4d PI A/J and C57BL/6 mice.

Figure 2.9: IL-12/23p40, TNF- $\alpha$, CXCL10, and CCL5 production in cells from 90d PI $\mathrm{A} / \mathrm{J}$ and $\mathrm{C} 57 \mathrm{BL} / 6$ mice .70

Figure 2.10: CXCL1, CCL2, and IL-6 production in cells from 90d PI A/J and C57BL/6 mice. 
Figure 3.1: Weight gain in H. bilis- and sham-infected $\mathrm{Smad} 3^{-/-}$mice.

Figure 3.2: Gross and histological appearance of mucinous adenocarcinoma in colon of

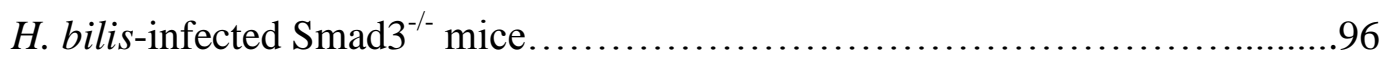

Figure 3.3: Incidence of neoplasia and hyperplasia in $\mathrm{Smad}^{-{ }^{--}}$mice.

Figure 3.4: Appearance of mucinous adenocarcinoma in situ and on microMRI in $H$. bilis-infected $\mathrm{Smad}^{-/-}$mice.

Figure 3.5: Copies of 18s rRNA gene in feces of H. bilis- and sham-infected Smad3 $3^{-/-}$ mice. .99

Figure 3.6: Expression of IL-1 $\beta$, MIP-1 $\alpha$, RANTES, and MCP-2 in feces of $H$. bilis- and sham-infected $\mathrm{Smad}^{-/-}$mice at nine weeks PI

Figure 3.7 Expression of IL-1 $\beta$, MIP-1 $\alpha$, and RANTES in feces of $H$. bilis- and shaminfected $\mathrm{Smad}^{-/-}$mice at weeks one to seven PI. .101

Figure 3.8: Correlation of lesion scores at nine weeks PI to fecal expression of IL-1 $\beta$, MIP-1 $\alpha$, and RANTES at weeks one to seven PI......................... 102

Figure 3.9: Receiver operating characteristic curves for fecal expression of IL-1 $\beta$, MIP$1 \alpha$, and RANTES at weeks one to five PI. .103

Figure 4.1: Weight gain in murine norovirus- and sham-infected BALB/c and C57BL/6 mice treated with DSS. 117

Figure 4.2: Histological appearance of lesions in DSS-treated C57BL/6 mice. 118 
Figure 4.3: Lesion scores in murine norovirus- and sham-infected BALB/c and C57BL/6 mice treated with DSS .............................................119

Figure 4.4: Weight gain in murine norovirus- and sham-infected SJL/J mice treated with

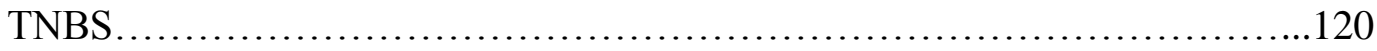

Figure 4.5: Kaplan-Meier survival curve of murine norovirus- and sham-infected SJL/J mice treated with TNBS .................................................. 121

Figure 4.6: Histological appearance of lesions in TNBS-treated SJL/J mice...........122

Figure 4.7: Lesion scores in murine norovirus- and sham-infected SJL/J mice treated with TNBS.................................................... 123 


\section{LIST OF TABLES}

Table 3.1: Sense and antisense primers, expected product sizes, and annealing temperatures used for real-time polymerase chain reaction amplification......105 


\section{LIST OF ABBREVIATIONS}

The following list contains abbreviations used throughout the dissertation.

ANOVA: analysis of variance

AOM: azoxymethane

APC: adenomatous polyposis coli or allophycocyanin

ATG16L1: autophagy-related protein 16L1

AUC: area under the curve

Batf3: basic leucine zipper transcriptional factor ATF-like 3

CAC: colitis-associated colorectal cancer

CARD15: caspase recruitment domain 15

CCL5: systematic name for RANTES

CD: Crohn's disease or cluster of differentiation

CFU: colony-forming unit

CIMP: $\mathrm{CpG}$ island methylator phenotype

CIN: chromosomal instability

COX: cyclooxygenase

CRC: colorectal cancer

CT: computed tomography

CXCL1: systematic name for $\mathrm{KC}$

CXCL10: systematic name for IP-10

$\mathrm{CX}_{3} \mathrm{CR} 1$ : systematic name for fractalkine receptor

DC: dendritic cell

DEPC: diethylpyrocarbonate

DN: double negative, i.e. $\mathrm{CD} 8 \alpha^{-} \mathrm{CD} 11 \mathrm{~b}^{-}$

DP: double positive, i.e. $\mathrm{CD} 8 \alpha^{+} \mathrm{CD} 11 b^{+}$ 
DNA: deoxyribonucleic acid

dNTP: deoxyribonucleotide triphosphate

DSS: dextran sulfate sodium

DTT: dithiothreitol

EDTA: ethylenediaminetetraacetic acid

ELISA: enzyme-linked immunosorbent assay

ENU: ethylnitrosourea

FAP: familial adenomatous polyposis

FBS: fetal bovine serum

FITC: fluorescein isothiocyanate

FOBT: fecal occult blood test

Gai2: G protein $\alpha \mathrm{i} 2$

G-CSF: granulocyte colony-stimulating factor

GIT: gastrointestinal tract

GM-CSF: granulocyte macrophage colony-stimulating factor

GWAS: genome-wide association study

HBSS: Hank’s balanced salt solution

HEPES: 4-(2-hydroxyethyl)-1-piperazineethanesulfonic acid

HNPCC: hereditary non-polyposis colorectal cancer

HPF: high-power field

HPRT: hypoxanthine-guanine phosphoribosyltransferase

HSP: heat shock protein

IBD: inflammatory bowel disease

IFN- $\gamma$ : interferon- $\gamma$

IL: interleukin 
IP-10: interferon gamma-induced protein 10

ITF: intestinal trefoil factor

$\mathrm{KC}$ : keratinocyte chemoattractant

LPS: lipopolysaccharide

LRR: leucine-rich repeat

MAP: mitogen-activated protein or Mycobacterium avium subsp. paratuberculosis

MCP-2: monocyte chemotactic protein-2

MDP: muramyl dipeptide

Mdr: multi-drug resistance

MFI: microbead fluorescent immunoassay

MIG: monokine induced by gamma interferon

MIP-1 $\alpha$ : macrophage inflammatory protein- $1 \alpha$

MNV: murine norovirus

MRI: magnetic resonance imaging

mRNA: messenger ribosomal nucleic acid

MSI: microsatellite instability

MUC: mucinous adenocarcinoma

NF- $\mathrm{B}$ : nuclear factor-kappa B

Nod2: nucleotide oligomerisation domain-containing protein 2

PCR: polymerase chain reaction

PE: phycoerythrin

PFU: plaque-forming unit

PRR: pattern recognition receptor

QTL: quantitative trait loci

PBS: phosphate-buffered saline 
PI: post-inoculation

Rag2: recombinase-activating gene 2

RANTES: regulated and normal T cell expressed and secreted

ROC: receiver-operator characteristic

RPMI: Roswell Park Memorial Institute

rRNA: ribosomal ribonucleic acid

RT-PCR: reverse transcription polymerase chain reaction

SA-PE: streptavidin-phycoerythrin

SCC: sporadic colorectal cancer

Smad3: Sma- and mothers against decapentaplegic homolog 3

SPF: specific pathogen-free

SROC: Spearman's rank order correlation

TCR: T cell receptor

TGF- $\beta$ : transforming growth factor- $\beta$

TLR: Toll-like receptor

TMB: 3,3',5,5'-tetramethylbenzidine

TNBS: 2,4,6-trinitrobenzene sulfonic acid

TNF- $\alpha$ : tumor necrosis factor- $\alpha$

TNFAARE: tumor necrosis factor delta AU-rich element

UC: ulcerative colitis

WT: wild type 


\begin{abstract}
Inflammatory bowel disease (IBD) is one of the most common immune-mediated diseases in the United States costing the average patient tens of thousands of dollars annually, and greatly diminishing quality-of-life. While there is no universal cure for IBD, recently developed treatments targeting the immunological basis of disease have proven successful in managing clinical symptoms. However, these pharmaceutical therapies such as infliximab (Remicade®) carry several side effects and are not efficacious in all patients. Thus, more selective treatments are needed. One necessary step in the development of such agents is a more precise understanding of which cells in the gastrointestinal tract are primary contributors to the pathogenesis of IBD.
\end{abstract}

We demonstrate that a rare subset of dendritic cells expressing CD8 $\alpha$ is present in significantly different numbers in mouse strains considered susceptible or resistant to a microbially induced model of IBD. Additionally, we show that cells derived from the target organ of susceptible mice prior to and shortly after induction of the disease process are prone to production of greater levels of certain inflammatory mediators including IL12/23p40, IP-10, RANTES, and TNF- $\alpha$. Lastly, we describe the generation of a mouse strain susceptible to the disease model but selectively lacking the subset of dendritic cells expressing CD8 $\alpha$, to be used in future studies.

One of the most serious sequela to IBD is colitis-associated colorectal cancer (CAC). Diagnosis of colorectal cancer in general is reliant on tests that suffer from either poor sensitivity or specificity (such as fecal occult blood tests), or invasiveness (such as colonoscopy). Newer genetic tests have been developed for the identification of 
hereditary risk factors, however CAC follows a molecular pathway distinct from that of familial forms of colorectal cancer. Thus, the development of noninvasive screening assays for CAC with high sensitivity and specificity would increase compliance with screening recommendations and enhance detection and survival of patients with CAC.

We report here the development of a novel screening technique capable of detecting the earliest stages of CAC in a microbially induced mouse model. Using fecal gene expression-based biomarkers, rRNA derived from colonocytes sloughed in the feces, we were able to accurately predict which mice would develop CAC several weeks later, as well as predict the severity of disease. While the optimal markers of disease identified in the present studies, IL-1 $\beta$ and MIP-1 $\alpha$, are likely specific to this model of CAC, the proof-of-concept portends a powerful new method of diagnostics for CAC in humans. 


\section{CHAPTER 1}

\section{INTRODUCTION}

The human gastrointestinal tract (GIT) is an organ faced with unique challenges and, in most individuals, endowed with the capacity to handle those challenges. The GIT is essentially a long tube in direct contact with the external environment at either end, i.e. the mouth and anus, constituting the largest surface area in the body exposed to bacteria ${ }^{1}$. A single layer of epithelial cells connected to each other via tight junctions is the only thing separating the myriad bacteria within the lumen of the GIT from underlying cells and tissues. Among those cells beneath the intestinal epithelium are various types of dendritic cells capable of extending pseudopodia between epithelial cells in a tightjunction-mediated manner and sampling the intestinal microbiota. Additionally, specialized M-cells overlying lymphoid follicles in the intestinal mucosa transcytose bacteria to underlying antigen-presenting cells. These cells, each possessing surface molecules capable of detecting pathogenic and non-pathogenic microbes, must be able to determine the correct response to the microbes within the GIT and direct the immune response accordingly via production of cell-surface co-stimulatory molecules and secreted molecules such as cytokines and chemokines. Within the lumen of the GIT resides a dynamic ecological community with a richness and diversity comparable to any environmental ecological niches known to man. This complex community of microbes consisting of members of all three biological domains, Bacteria, Archaea, and Eukarya, is

dominated by eubacteria with densities of greater than $1 \times 10^{11} /$ gram of feces in the colon. In an average adult, prokaryotes in the GIT outnumber somatic cells by an order of magnitude. The composition of this dense and diverse population is largely stable in 
healthy adults but, as in any population of this size, it is dynamic and may experience fluctuations in the relative frequency of specific microbial species from time to time. This microbiota is recognized by the innate immune system which, in turn, directs the host response. The studies described in the following dissertation investigate the contribution of $\mathrm{CD} 8 \alpha^{+}$dendritic cells in the pathogenesis of a model of inflammatory bowel disease (Chapter 2), the noninvasive detection of colitis-associated colorectal cancer in a microbially induced model (Chapter 3), and the impact of acute infection with Murine Norovirus on two chemically induced models if inflammatory bowel disease (Chapter 4). It is the goal of the author that the findings detailed herein will advance the field of gastroenterology and immunology in such a fashion as to lead us one step closer to more effective therapeutic and diagnostic techniques in humans.

\section{SIGNIFICANCE OF INFLAMMATORY BOWEL DISEASE}

Inflammatory bowel disease (IBD) is one of the most common chronic immune-mediated diseases in the Western world affecting approximately 1.4 million people in the U.S. and close to 3 million people in Europe ${ }^{2}$. In addition, the incidence and prevalence of IBD is increasing worldwide, particularly in countries with a Western lifestyle. Traditionally, a diagnosis of IBD is classified as either ulcerative colitis (UC) or Crohn's disease (CD) although there is considerable variation in clinical disease presentation and histological appearance of diseased tissues within either form of IBD. Additionally, approximately $10 \%$ of IBD cases not meeting the criteria for a diagnosis of UC or CD are deemed indeterminate colitis. Clinical manifestations of IBD are typically waxing and waning and most commonly include diarrhea or loose stool, constipation, painful abdominal cramping, hematochezia or melena depending on the anatomical location of ulcerated 
areas, and the sensation of incomplete evacuation. General symptoms associated with IBD include fever, loss of appetite, fatigue, weight loss, and a loss of normal menstrual cycles in females.

While there are overlapping causative factors in $\mathrm{UC}$ and $\mathrm{CD}$, there are distinct phenotypic differences. As the name implies, UC is restricted to the colon (and occasionally the rectum) while sparing the perianal region. Alternatively, CD can affect any region of the gastrointestinal tract from the mouth to the anus and perianal involvement is common. Affected areas are continuous in UC while so-called "skip areas" of unaffected mucosa are common in CD. The depth of inflammation also varies between the two forms of IBD with substantial consequence; while histological examination of UC reveals inflammation extending only into the lamina propria, the inflammatory infiltrates in CD are frequently transmural, leading to fistulae necessitating surgical repair. Similarly, while strictures and granulomata are common in $\mathrm{CD}$, these are seen only occasionally in UC patients.

The inflammation and subsequent damage to the intestinal mucosa may also lead to significant sequelae, some of which are as, if not more, problematic than IBD itself. One of the most common consequences of IBD is nutritional deficiency, with protein, iron, and vitamin $\mathrm{D}$ being some of the most commonly deficient components ${ }^{3-5}$. Other factors shown to be lacking in IBD patients include magnesium, and vitamins $\mathrm{B}_{9}$ and $\mathrm{B}_{12}$. Not surprisingly, these are primarily a problem of $\mathrm{CD}$ patients with small intestinal involvement; however malnutrition can affect patients with any form of IBD even when disease is quiescent ${ }^{3}$. There are several factors leading to malnutrition including malabsorption, lack of appetite, increased losses in feces, and the overall shift in 
metabolic function due to chronic inflammation. Of note, malnutrition often leads to its own set of health issues including poor wound healing, anemia, osteoporosis, and, in case of pediatric IBD, stunted growth. One of the most devastating sequelae to IBD is development of colitis-associated colorectal cancer (CAC), thought to be induced by the abundant reactive oxygen species produced by activated cells of the immune system, as well as the increased rate of epithelial cell turn-over in the gut. Along with hereditary predispositions such as Familial Adenomatous Polyposis (FAP) and Hereditary NonPolyposis Colorectal Cancer (HNPCC), chronic inflammation is one of three primary risk factors for colorectal cancer. CAC is however distinct from sporadic and familial forms of colorectal cancer with regard to its molecular pathogenesis and phenotypic progression. These topics will be reviewed further beginning on page 19 .

IBD can affect individuals of almost any age and, while the incidence of pediatric IBD is increasing, disease is most often diagnosed during late adolescence or early adulthood. A diagnosis of $\mathrm{CD}$ carries a relatively modest increase in mortality while UC appear to affect mortality to a lesser degree ${ }^{6,7}$. Additionally, while partial or total colectomy may be curative in rare cases of UC, the majority of IBD cases must be managed for the life of the patient making IBD as costly to the individual and the health care industry as other chronic diseases such as congestive heart failure and cancer ${ }^{8}$. Costs associated with IBD include medications, hospitalization, and surgery, as well as lost wages due to disability which account for almost $50 \%$ of total $\operatorname{costs}^{9-11}$. The recent advent of effective but expensive biologic therapies such as anti-tumor necrosis factor (TNF)- $\alpha$ have shifted the profile of these costs via a decrease in costs related to hospitalization and surgery and a concomitant increase in costs related to pharmaceuticals ${ }^{12}$. Health care costs tend to be 
greatest during the initial few years after the diagnosis of IBD is made followed by a plateau at slightly lower costs, with lifetime mean direct costs of $\$ 4232$ and $\$ 3552$ annually for Canadian patients with $\mathrm{CD}$ and $\mathrm{UC}$ respectively ${ }^{13}$. A recent U.S. study determined that patients with mild IBD incurred average annual health care costs of $\$ 10,687$ whereas patients with moderate to severe IBD had average annual costs of $\$ 37,925^{8}$. These figures do not account for indirect costs which are generally considered to be equal to, or greater than, direct costs, due to the facts that IBD generally occurs in the most economically productive years of life, and that it is typically a nonfatal disease requiring life-long management ${ }^{14,15}$. Additionally, as the prevalence of IBD in the U.S increases, so do health care costs; inflation-adjusted total charges attributable to CD hospitalizations increased an average of $10.5 \%$ annually between 1998 and $2004^{16}$.

Along with the physical and financial burden placed on individuals diagnosed with IBD, there is a tremendous psychological burden. Individuals living with chronic illnesses such as IBD report feelings of diminished self-worth, shame, and embarrassment. This sense of stigma is often perceived to exist in health care settings and, whether real or not, can lead to delayed health care and decreased quality of life ${ }^{17}$. IBD can also lead to depression and anger due to the impact of IBD on a patient's lifestyle such as dietary changes, cessation of smoking, or loss of productivity. One recent study of IBD patients demonstrated a significant correlation between the level of depression and the number of relapses experienced ${ }^{18}$. Thus, the negative effect of IBD on mood and self-worth can in turn exacerbate disease leading to a vicious cycle. 


\section{PATHOGENESIS OF INFLAMMATORY BOWEL DISEASE}

The pathogenesis of IBD is complex and multifactorial. Susceptibility is determined by a combination of genetics and multiple environmental factors including, but not limited to, geography, diet, smoking, and stress. Researchers have long known that genetics played a large role in disease susceptibility based on studies of familial cohorts ${ }^{19,20}$ and twins ${ }^{21-}$ 24. Overall, estimates of the contribution of genetics to disease susceptibility are approximately $50 \%^{25,26}$. Genome-wide association studies (GWAS) have identified several genes linked to IBD susceptibility and resistance ${ }^{27,28}$, many of which are associated with the innate immune system and intuitively involved in the pathogenesis of IBD. Using standard linkage analysis rather than a GWAS, the first genetic polymorphisms associated with IBD occurred in the gene encoding Nucleotide oligomerisation domain-containing protein 2 or Nod2 (also known as Caspase Recruitment Domain 15 or CARD15) ${ }^{29-31}$. Expressed primarily in leukocytes and Paneth cells $^{32}$, Nod2 is an intracellular protein which recognizes muramyl dipeptide (MDP) ${ }^{33}$, a peptidoglycan component of both Gram positive and Gram negative bacteria. The Cterminal of Nod2 contains a leucine-rich repeat (LRR) domain which physically binds MDP, while the N-terminal portion contains two caspase-recruitment (CARD) domains capable of initiating apoptosis and activating inflammatory NF- $\mathrm{kB}$ pathways ${ }^{34}$. Three principal mutations have been identified in the Nod2 gene conferring increased susceptibility to IBD (Arg702Trp, Gly908Arg, and Leu1007fs) ${ }^{35}$ and all three occur in the region encoding the LRR domain, implying that these mutations affect the ability of Nod2 to bind MDP normally. Highlighting the contribution of other genetic and environmental factors however, these same mutations do not appear to predispose some 
Asian populations to $\mathrm{IBD}^{36,37}$. Additionally, while homozygous and compound heterozygous Nod 2 mutations are estimated to increase the risk of IBD from 10 to 40 times the general population, a substantial proportion of healthy people also possess those genotypes $^{38}$. Considering its location in the cytosol, the physiological function of Nod2 is presumably to recognize intracellular bacteria. While acute signaling through Nod2 initiates NF- $\kappa \mathrm{B}$ and MAP kinase-driven inflammatory responses ${ }^{39}$, chronic stimulation of Nod2 leads to a tolerogenic response ${ }^{40}$, suggesting that the chronicity of binding also affects the ensuing immune response. Thus, individuals possessing mutated forms of Nod2 may be unable to mount proper immune responses against invasive microbial species, or to induce tolerance to commensal microbes collected from the intestinal lumen via $\mathrm{CX}_{3} \mathrm{CR} 1-$ mediated sampling by dendritic cells. Nod2 has also been shown to work synergistically with other pathogen-associated molecular pattern receptors such as Toll-like receptor 2 (TLR2), TLR3, and TLR $4^{41,42}$. Thus, like TLRs, Nod 2 can serve as an inducer of inflammatory or tolerogenic responses. As an example of the latter, the beneficial effects of certain lactobacilli have been shown to be mediated through $\operatorname{Nod} 2^{43}$. Perhaps not surprisingly, Nod2 is recognized to play a critical role in modulating the intestinal microbiota ${ }^{44}$ via modulating the response of $\mathrm{T}$ cells, the production of antimicrobial defensins ${ }^{45,46}$, and the permeability of the intestinal epithelium ${ }^{47}$.

GWAS have identified other pathways associated with IBD susceptibility including autophagy and several inflammatory cytokines and cytokine receptors. Autophagy, once considered strictly a process by which intracellular organelles can be degraded and their components recycled, is now recognized to also serve as a means of eradicating intracellular microbes. In the process of autophagy, cytoplasmic material is engulfed in 
membrane-enclosed autophagosomes which then fuse with endosomes and lysosomes to form autophagosomes in which degradation occurs. The first component of this process to be associated with IBD was Autophagy-related protein 16-L1 (ATG16L1) ${ }^{48-50}$, a subunit of the large protein complex necessary for autophagy. Subsequently, variants of the protein Immunity-related GTPase M (IRGM), essential for the initiation phase of autophagy and the clearance of certain intracellular microbes ${ }^{51,52}$, have been linked to IBD susceptibility ${ }^{53}$. Surprisingly, two recent reports suggest that Nod2 may regulate autophagy and that Nod2 and ATG16L1 may even physically interact ${ }^{54,55}$. The nature of this interaction is not completely understood and is an active area of research. Cytokines implicated in the pathogenesis of IBD include IL-23, IL-10, IL-1 $\beta$, IL-12, IL-27, IL-2, and TNF- $\alpha$, among others. Additionally, multiple chemokines affecting leukocyte recruitment to the gut, mucin proteins contributing to epithelial barrier function, and transcription factors involved in myriad pathways have been associated with the risk of developing IBD, all of which speak to the remarkable complexity of the pathogenesis of IBD.

Despite the number of genetic factors now linked to disease susceptibility, the exact nature of IBD pathophysiology is still largely unknown. Attempts to identify a pathogenic microbial etiology have been, as a whole, unfruitful. The histological and endoscopic appearance of $\mathrm{CD}$ bears a resemblance to infection with Mycoplasma tuberculosis and Yersinia spp. while acute UC has strong similarities to Shigella and Campylobacter infections ${ }^{56}$. That being said, none of these pathogens have been consistently recovered from affected patients. One bacteria receiving considerable attention as a possible causative agent is Mycobacterium avium subspecies 
paratuberculosis (MAP), the cause of Johne's disease, a chronic granulomatous enteritis of cattle. MAP has been recovered from several CD patients and increased antibody titers against certain mycobacterial antigens have been noted. However, immunohistochemical and clinical studies have failed to support the notion that MAP is causative in $\mathrm{IBD}^{57,58}$. Additionally, PCR-based attempts to support the role of MAP in IBD have shown that while sequences specific for mycobacteria can be isolated from intestinal tissue, they are not specific for $\mathrm{CD}^{57,58}$. Others have proposed that in utero or childhood exposure to one of several viral pathogens may lead to CD years later. Viruses hypothesized at one point to play a role include the paramyxoviruses responsible for the measles and mumps ${ }^{59,60}$, and Epstein-Barr virus ${ }^{61}$ and cytomegalovirus ${ }^{62}$, both human herpesviruses. As with the bacterial agents mentioned above, these hypotheses have been discredited as causative agents ${ }^{63-65}$ and any weak positive associations between childhood infection with, or vaccination against, these agents are now considered, at best, environmental factors contributing to IBD risk. Thus barring the identification of an as yet uncharacterized intestinal microbe more strongly associated with IBD, there is no convincing evidence that IBD is caused by a single bacteria or virus. Additionally, even in the unlikely event that such a microbe is identified, the contribution of genetic risk factors is irrefutable.

There has also been considerable investigation into IBD being an autoimmune disease targeting a host protein, particularly in $\mathrm{UC}^{66-69}$. Anti-neutrophil antibodies have been studied extensively, with conflicting results. While elevated anti-neutrophil titers have been detected in some IBD patients ${ }^{70,71}$, their presence is not diagnostic of IBD and titers do not correlate with disease activity in $\mathrm{UC}^{72}$. One theory, termed molecular mimicry, 
holds that specific bacterial epitopes share immunological determinants with host proteins. One example posits that the mycobacterial heat shock protein heat shock protein 65 (HSP65) shares sufficient homology with human HSP60 to induce an autoimmune response. These findings have achieved little support. Thus, the endogenous antigens, be they microbial or host-associated, remain elusive.

In contrast to hypotheses revolving around immune responses to an unidentified pathogenic organism or host epitope, the commonly accepted theory is that IBD results from an aberrant immune response against some component of the commensal microbiota. This notion is supported in animal models of IBD, almost none of which develop intestinal inflammation when performed in germ-free animals. In the human condition, there are several pieces of evidence suggesting that autochthonous bacteria are the target of the inflammatory immune responses in IBD. Clinically, treatment with antibiotics or surgical diversion of the fecal stream has shown to have positive effects in many CD patients. Histological analysis has revealed that the mucosa is relatively free of adherent bacteria in the normal GIT $^{73}$. In IBD patients however, there is an increased presence of bacterial antigens within the mucosa, including antigens derived from E. coli, Streptococcus spp., and Listeria $\operatorname{spp}^{73-76}$. However, while immunoreactivity is most commonly localized to fissures, granulomatous tissue, and abscesses, it is unclear whether these agents are causally related or opportunistic. Similarly, studies have demonstrated increased levels of circulating antibodies against intestinal bacteria ${ }^{77,78}$, the significance of which is yet unknown. A prevailing theory, supported by studies using animal models holds that certain bacterial species function as provocateurs of immune responses against commensal bacteria by compromising the mucosal barrier function. 
Using a mouse model driven by experimental inoculation with Helicobacter bilis or $H$. hepaticus, Wannemuehler et al. showed that several weeks after inoculation, antibodies against commensal bacteria consistently appeared in mice, despite any overt defects in the mucosal lining, e.g. ulceration ${ }^{79,80}$. Supporting this theory in humans, Rocha et al. demonstrated the ability of $E$. coli to enhance the permeability of cultured epithelial cell monolayers ${ }^{81}$. It is unknown how the supposed provocateurs induce this alteration in permeability and there is considerable work to be done in this field. Collectively, these data support the hypothesis that IBD results from a dysregulated immune response to commensal bacteria.

\section{ANIMAL MODELS OF INFLAMMATORY BOWEL DISEASE}

While no animal model of IBD recapitulates every aspect of the human condition, such reductionist approaches provide important information on the development of disease and factors predisposing individuals to disease. Owing to our ability to manipulate the mouse genome, mice are the most widely used model species in most fields of biomedical research and studies of IBD are no exception. Inflammation is induced in these models via oral or rectal administration of chemical compounds, genetic alteration, microbial inoculation, or adoptive transfer of cells.

The two most commonly used chemically induced mouse models of IBD are DSS- and TNBS-induced colitis. In DSS colitis, the causative agent dextran sulfate sodium, a highly sulfated polysaccharide of very high molecular weight (typically 5 to $40 \mathrm{kDa}$ ), is administered in the drinking water. First developed by Ohkusa et al. in hamsters ${ }^{82}$, the technique was soon translated to mice ${ }^{83}$ and has become a classic method of inducing 
colitis and potentially colorectal cancer when administered chronically in susceptible strains of mice. Disease severity is dependent on multiple factors including mouse $\operatorname{strain}^{84}$, mouse sex, molecular weight of $\mathrm{DSS}^{85}$, duration of exposure to $\mathrm{DSS}^{86}$, and even the stress level of mice ${ }^{87}$. In the GIT, DSS penetrates the mucosal epithelium and can be visualized within antigen-presenting cells in the colon and mesenteric lymph nodes within 24 hours of the first dose ${ }^{88}$. Acute colitis can be induced in susceptible mouse strains by continuous administration of $2-5 \%$ DSS for periods as short as 4 days. Chronic colitis is induced via prolonged administration of lower concentrations or by cyclical dosing. Histologically, DSS induces an initial loss of crypts followed by epithelial ulceration and abscessation ${ }^{89}$. Thus DSS colitis is often described histologically as suppurative colitis with ulceration. Despite the overt toxicity of DSS to colonic epithelial cells, the intestinal microbiota is essential for the development of inflammation ${ }^{90}$, indicating the necessity of the microbiota for disease.

2,4,6-trinitrobenzene sulfonic acid (TNBS) is used to induced colitis via rectal administration in approximately $50 \%$ ethanol. As in DSS colitis, there are differences in strain susceptibility. In susceptible strains, greater than $90 \%$ of TNBS-treated mice will develop acute colitis followed by weight loss and diarrhea within days to weeks. The age of mice is a critical factor as mice less than 4 weeks of age will experience excessive mortality whereas mice greater than 8 weeks of age will develop much milder, if any, disease $^{91}$. Additionally, proper storage and dosage of TNBS are important considerations. It is thought that the trinitrophenyl moiety of TNBS haptenates host proteins, and also evokes a cross-reactive immune response, cellular elements of which recognize portions of the microbiota. This was supported by experiments by Neurath et 
al. demonstrating that disease can be transferred with $\mathrm{T}$ cells extracted from the lamina propria of TNBS-treated mice to naïve mice in the absence of $\mathrm{TNBS}^{92}$. Cytokine profiles from TNBS-treated mice indicate that inflammation is driven by $\mathrm{T}_{\mathrm{H}} 1$-skewed $\mathrm{CD} 4^{+} \mathrm{T}$ cells $^{93}$, similar to IBD in humans ${ }^{94,95}$. Histologically, TNBS administration leads to dense transmural mononuclear cell infiltration and a loss of normal crypt architecture.

A detailed description of genetically manipulated murine models of IBD is beyond the scope of this section. It suffices to say that these models, including but not limited to IL$2^{-/-96}, \mathrm{G} \alpha 2^{-/-97}, \mathrm{TCR} \alpha^{-/-98}, \mathrm{TCR} \beta^{-/-99}, \mathrm{Mdr}^{-/-100}, \mathrm{MUC}^{-/-101}, \mathrm{ITF}^{-/-102}, \mathrm{TNF} \Delta \mathrm{ARE}{ }^{103}$, and Stat4-transgenic ${ }^{104}$ mice all possess strengths and weaknesses. Of note however is the well-characterized IL- $10^{-/-}$mouse, originally developed by Kühn et $a l^{105}$. In the absence of IL-10, an immunosuppressive cytokine, or a functional IL-10 receptor $^{106}$, mice develop intestinal inflammation. In specific pathogen-free (SPF) conditions, inflammation is mild, restricted to the colon, and does not manifest until several months of age. In conventionally housed mice however, inflammation is much more robust, develops more rapidly, and affects the upper and lower GIT. Additionally, IL-10 ${ }^{-/-}$mice raised in conventional conditions develop several extraintestinal complications such as anemia, splenomegaly, and hepatitis ${ }^{107}$. Considering the impact of housing conditions, IL-10 ${ }^{-/}$ mice have been used to identify which particular microbial species are responsible for the differences in the development of inflammation. Kullberg et al. demonstrated that $H$. hepaticus is largely responsible for the exacerbated disease in conventionally housed mice $^{108}$. Interestingly, $H$. hepaticus-infected $\mathrm{IL}-10^{-/-}$mice raised in otherwise SPF conditions still fail to develop inflammation of the upper GIT. The microbes responsible for this phenomenon in conventionally housed mice is unknown ${ }^{107}$. The $\mathrm{T}$ cell- 
dependent ${ }^{109}$ inflammation in IL-10 $0^{-/-}$mice was originally reported to be skewed toward a $T_{H} 1$ phenotype ${ }^{110}$, although studies performed subsequent to the discovery of the $T_{H} 17$ pathway have provided evidence that, in the absence of IL-10 signaling, IL-23 also plays a role in $H$. hepaticus-induced colitis ${ }^{111}$. Thus, it is thought that the $\mathrm{T}_{\mathrm{H}} 1$ and $\mathrm{T}_{\mathrm{H}} 17$ pathways, both of which are regulated by IL- $10^{112,113}$, function synergistically to promote T cell-dependent $H$. hepaticus-driven intestinal inflammation.

The essential nature of IL-10 in the regulation of intestinal inflammatory responses has led to extensive study of regulatory T cells in IBD. Toward that end, adoptive transfer of $\mathrm{T}$ cell subsets into scid mice has become another widely used model of IBD. In this model, effector T cells, defined by high expression of CD45RB, induce colitis in severely immunodeficient (scid) mice when administered intravenously. Alternatively, co-transfer of CD45RB ${ }^{\text {low }} \mathrm{T}$ regulatory cells is capable of suppressing this inflammation. Antibody neutralization studies have revealed that the immunosuppressive capacity of these cells is dependent on both IL-10 and TGF- $\beta$. While it has not been explicitly reported as such, a review of the literature suggests that the transfer recipient must be infected with Helicobacter for the development of robust inflammation. Thus, as in $\mathrm{IL}-10^{-/-}$mice, while $H$. hepaticus is not absolutely required for the development of inflammation, it dramatically enhances the rapidity and severity of disease.

Almost all mouse models of IBD require intestinal microbiota for the development of optimal disease ${ }^{114-118}$. While the addition of $H$. hepaticus often exacerbates these models ${ }^{119-122}$, disease is nonetheless dependent on the presence of a background microbial population. Similarly, the introduction of certain Helicobacter spp. into some wild-type mice with an established microbiota is capable of inducing inflammation, as in $\mathrm{A} / \mathrm{J}$ mice. 
However, IL-10 $10^{--}$mice fail to develop intestinal inflammation when mono-associated with $H$. hepaticus, showing the resident microbiota is essential in the induction of disease even in a host genetically predisposed to intestinal inflammation ${ }^{123}$. These contradictions in the pathogenicity of Helicobacter spp. may be explained by recent studies demonstrating that Helicobacter spp. may act as "provocateurs" of immune responses against the background microbiota. In the presence of $H$. bilis, gnotobiotic mice generate robust IgG responses against several members of a defined microbiota ${ }^{79,80}$ supporting the notion that, while Helicobacter is itself pathogenic in susceptible strains of mice, the inflammation is partially in response to other microbial species as well.

A weakness of all of the aforementioned models of IBD is the nature of the disease induction. Models are either performed in a selectively or globally immunodeficient mouse strain, or are induced via administration of a synthetic compound not encountered in nature. Thus, an immunocompetent mouse model of IBD would provide a useful means of dissecting the cellular and molecular factors contributing to disease susceptibility. Also, the majority of mouse models of IBD are dependent on the presence of the adaptive immune system ${ }^{91}$. As there is considerable evidence that IBD in humans has a basis in the recognition of microbes by cells of the innate immune system, a model of IBD that functions independent of the adaptive immune system is desirable. Lastly, in an ideal model of IBD, inflammation would manifest in response to commensal intestinal microbes and would have phenotypic similarities to IBD in humans. All of these criteria are met in the $H$. hepaticus-infected A/J mouse model of IBD.

A/J and B6 mice are both immunocompetent strains of mice, with demonstrated differential susceptibility to $H$. hepaticus-mediated cecal inflammation. The lack of any 
genetic manipulation provides a more realistic comparison to human physiology. In contrast to most other mouse models of IBD, $H$. hepaticus-induced inflammation in $\mathrm{A} / \mathrm{J}$ mice is independent of an adaptive immune system. While the histological appearance of disease is clearly (and not surprisingly) altered in A/J mice lacking functional $\mathrm{T}$ and $\mathrm{B}$ cells, disease persists nonetheless. Additionally, the absence of disease in H. hepaticusinfected B6 mice is also independent of an adaptive immune system. Thus, as the innate immune system is increasingly implicated in the pathogenesis of IBD, the H. hepaticusinfected $\mathrm{A} / \mathrm{J}$ model offers a model of IBD driven by the innate immune system and its recognition of the gut microbiota. This is critical in the proceeding studies as published reports of the role of various subsets of intestinal dendritic cells (DC) in inflammation have relied on in vitro stimulation using pathogens associated with disease in other organ systems $^{124}$, or highly artificial adoptive transfer models ${ }^{125}$. Other investigations of the function of intestinal DC subsets have examined physiological settings in the absence of inflammation $^{126-130}$. Thus, there is a distinct lack of knowledge regarding the role of intestinal DC subsets in IBD-like syndromes.

As mentioned above, there is also convincing evidence that multiple bacterial species closely related to $H$. hepaticus induce host immune responses against commensal members of the intestinal microbiota. Of note, antigen-specific IgG1 and IgG2a responses to commensal microbes were detected in $H$. bilis-infected mice, and mesenteric lymphocytes from $H$. bilis- and Brachyspira hyodysenteriae-infected mice produced higher levels of IFN- $\gamma$ than control mice ${ }^{79,80}$. The extended period of latency between experimental inoculation with $H$. hepaticus and the development of histologically identifiable inflammation in $\mathrm{A} / \mathrm{J}$ mice fits well with the concept of certain Helicobacter 
spp. functioning as provocateurs of immune responses against microbes not normally capable of inducing such reactions.

Lastly, there are considerable phenotypic similarities between $H$. hepaticus-infected A/J mice and humans affected with IBD. Histologically, the ceca of infected A/J mice possess mild to marked inflammation characterized by mononuclear cells (the majority of which have a large nuclear:cytoplasmic ratio consistent with lymphocytes), and mild to moderate hyperplasia. Inflammation is multifocal to continuous and may extend deep into the lamina propria but rarely into the muscularis. The immune response in infected A/J mice represents a generalized innate immune response, typified by increased expression of several chemokines and genes involved in antigen processing, followed by a $\mathrm{T}_{\mathrm{H}} 1$-driven adaptive immune response, characterized by increased expression of IFN- $\nu$ and multiple interferon-induced genes ${ }^{131}$. The immunopathogenesis of IBD is strikingly similar with altered expression of genes associated with the $T_{H} 1$ cytokine profile as well as more generalized acute inflammatory mediators such as TNF- $\alpha$. Interestingly, the $H$. hepaticus-infected A/J model also has certain attributes mimicking the demographics of human IBD. With respect to age, A/J mice infected at weaning develop disease at approximately four months of age. This corresponds to early adulthood, the age at which IBD is most commonly diagnosed in humans. Regarding sex, female A/J mice develop more severe inflammation than male mice, and express significantly higher levels of several cytokines and chemokines implicated in the disease process ${ }^{132}$. Similarly, there is a slight female predominance in Crohn's disease, especially among women in late adolescence and early adulthood ${ }^{133}$. Hence, the $H$. hepaticus-infected A/J mouse provides a uniquely applicable model of IBD with phenotypic and immunological 
similarities to IBD in humans, particularly in studies examining the innate immune system. 


\section{SIGNIFICANCE OF COLITIS-ASSOCIATED COLORECTAL CANCER}

Inflammation, characterized by the accumulation of activated leukocytes in affected tissue, occurs in response to tissue damage of almost any cause. In cases of acute and balanced inflammation, inflammation is beneficial to the host and serves to eradicate the insulting agent, and remove damaged cells and cellular components. Additionally, inflammatory cells stimulate wound-healing via promotion of angiogenesis, collagen deposition, and epithelial restitution, as well as proliferation or attraction of cells necessary for healing such as fibroblasts and platelets. Macrophages, a primary source of epidermal growth factor, platelet-derived growth factor, fibroblast growth factors 1 and 2 , transforming growth factors $\alpha$ and $\beta$, IL-1, and TNF- $\alpha$, are particularly critical for wound healing. In cases of acute inflammation, this process wanes as infectious agents are eradicated and the wound is repaired. If the causative agent is not removed however, chronic inflammation occurs, often to the detriment of the patient. In this situation, macrophages continually attempt to remediate tissue damage and, in the process, release abundant reactive oxygen and nitrogen species such as peroxides and peroxynitrite. These molecules lead to oxidative and nitrosative stress, which are also injurious to tissue when present at high levels or for prolonged periods. Additionally, chronic production of cytokines such as TNF- $\alpha$ and IL-1 can promote tumor growth via direct effects on tumor cells and stimulation of epithelial to mesenchymal transition ${ }^{134}$. Thus, chronic inflammation due to a persistent insult can lead to a vicious cycle of tissue damage and leukocyte recruitment, all of which leads to a pro-tumorigenic environment.

Chronic inflammation is associated with approximately $20 \%$ of human cancers ${ }^{135,136}$ and it is now well accepted that inflammation is causally linked to cancer ${ }^{137}$. This is 
especially germane to the GIT wherein conditions such as Barrett's esophagus ${ }^{138}$, infection with Helicobacter pylori ${ }^{139}$, and inflammatory bowel disease ${ }^{140}$ are primary risk factors for cancer of the affected organ. While colitis-associated colorectal cancer (CAC) constitutes a relatively small portion of colorectal cancer (CRC) in general, IBD patients are among the highest risk group for developing $\mathrm{CRC}^{141}$, with a two- to three-fold greater chance of developing colorectal cancer ${ }^{140}$. Critical factors affecting the risk of CAC include the duration, extent, and severity of inflammation ${ }^{2,140}$.

\section{PATHOGENESIS OF COLITIS-ASSOCIATED COLORECTAL CANCER}

CAC is distinct from familial forms of colorectal cancer and sporadic colorectal cancer (SCC) in both presentation and molecular pathogenesis. Clinically, CAC affects individuals at a younger age than SCC and there is a higher incidence of multiple synchronous primary lesions in CAC. The location also varies between the two forms of CRC with, on average, a more proximal location noted in CAC. Mucinous and "signet ring" phenotypes are also more frequently seen in CAC than in SCC, the significance of which remains unclear. Regarding the molecular events leading to neoplasia, SCC follows a characteristic progression from an adenomatous polyp driven by genetic and epigenetic alterations leading to the malignant transformation of colonic epithelium. The principal molecular pathways responsible for this transformation include chromosomal instability (CIN) and microsatellite instability (MSI), which account for approximately 85 and $15 \%$ of sporadic CRC respectively ${ }^{142}$, as well as epigenetic changes in the $\mathrm{CpG}$ island methylator phenotype (CIMP). CIN results in abnormal segregation of chromosomes and abnormal DNA content. The resulting loss of heterozygosity frequently leads to loss of function of tumor suppressor genes such as APC and p53, the 
former characteristically occurring early in disease progression, the latter much later. The MSI pathway is associated with loss of function of mismatch-repair genes which encode proteins responsible for the repair of DNA base-pair mismatches occurring during DNA replication. While MSI can thus lead to DNA replication errors throughout the entire genome, certain genes containing short nucleotide repeats that are intrinsically unstable (such as TGFßRII) are particularly susceptible to replication errors ${ }^{142}$. In contrast to SCC, CAC derives from an area of epithelial dysplasia rather than a polyp, and while CIN and MSI occur at roughly the same frequency as in SCC, molecular events follow a distinctly different progression (figure 1.1) ${ }^{142}$. For example, mutations in APC typically occur late in the development of $\mathrm{CAC}^{143,144}$, while mutations in $\mathrm{p} 53$, considered a key event in the progression from late adenoma to carcinoma in SCC, occur early in the development of $\mathrm{CAC}^{145}$.

While a patient with IBD and a family history of CRC has approximately a two-fold greater risk of developing CRC than an IBD patient with no family history of $\mathrm{CRC}^{146}$, no clear genetic etiology has been attributed to CAC per $s e^{142}$. Thus, as in IBD, there are likely environmental factors involved in disease risk as well. It is well-known that nonsteroidal anti-inflammatory drugs decrease the risk of SCC and polyps in individuals with familial adenomatous polyposis (FAP), presumably due to the inhibition of cyclooxygenase-2, a gene induced in premalignant colonic epithelium of IBD patients. Additionally, nutrition is thought to play a role in risk of CRC. In one cohort of patients affected with UC and demonstrating MSI in non-neoplastic colonic epithelium, folate levels were lower than in patients without MSI. Dietary supplementation of folate resulted in changes in the pattern of microsatellite stability. Thus, along with unknown 
genetic factors, medication and diet (and likely other environmental factors) also affect individual risk of CAC in IBD patients.

\section{ANIMAL MODELS OF COLITIS-ASSOCIATED COLORECTAL CANCER}

One of the most commonly used and oldest models of CRC is the APC ${ }^{\mathrm{min} /+}$ mouse, also referred to as the $\mathrm{APC}^{4716}$ or Min mouse ${ }^{147}$. These mice carry a heterozygous dominant mutation in the $A p c$ gene encoding a protein which modulates Wnt signal transduction through $\beta$-catenin, providing a model of FAP, a hereditary form of human CRC most often caused by mutations in Apc. These mice were developed via random mutagenesis using the alkylating agent ethylnitrosourea (ENU) and are maintained on a heterozygous background via matings between affected males and wild-type females as mice carrying the mutation have shortened life spans and affected females have difficulty maintaining pregnancies. Specific-pathogen-free $\mathrm{APC}^{\mathrm{min} / \mathrm{t}}$ mice develop multiple intestinal neoplasms (hence Min) throughout the small and large intestines, while germ-free mice experience a $50 \%$ reduction in tumor number leading to the model's use in studies of the role of intestinal microbiota on carcinogenesis ${ }^{148}$.

Regarding CAC, there are several mouse models including the $\mathrm{Ga}_{\mathrm{i} 2}{ }^{-{ }^{-}}, \mathrm{IL}-10^{-/}, \mathrm{IL}-2 / \beta_{2^{-}}$ microglobulin ${ }^{-1-}$, and Smad $3^{-/-}$strains of mice. Of note, many of these models fail to manifest disease unless infected, either naturally or experimentally, with certain enteric bacteria, typically Helicobacter hepaticus or H. bilis. As these Helicobacter spp. do not cause colorectal cancer in wild-type mice, these models provide the opportunity to study the combined contributions of the immune system and the intestinal microbiota in carcinogenesis. There are also chemically induced models of inflammatory bowel 
disease (IBD) which are potentially useful in the study of CAC, such as the dextran sodium sulfate (DSS) model, performed with ${ }^{149}$ or without ${ }^{150}$ the addition of the carcinogen azoxymethane (AOM). DSS, a high-weight sulfated polysaccharide, is administered in the drinking water and, due to its direct toxicity to crypt cells, results in a fairly acute loss of crypts, alterations in the microbiota, and inflammation with eventual development of CAC. There are multiple doses and schedules of DSS administration designed to model different human disease entities. In studies of CAC, it is commonly administered cyclically, e.g. 7 days on, 7 days off, etc., to mimic the remitting and relapsing nature of IBD. Additionally, inbred strains of mice vary in their susceptibility to this model ${ }^{151,152}$, allowing for studies of genetic susceptibility such as quantitative trait loci analyses. The addition of AOM results in more consistent and more acute development of tumors than that seen with DSS alone. Administration of AOM by itself also induces colorectal cancer in both rats and mice, potentially due to its effects on TGF$\beta$ signaling ${ }^{153,154}$, and has been used extensively in the study of potential protective compounds.

The model used in the studies described in Chapter 3 is the $\mathrm{Smad}^{-/-}$mouse on a $129 / \mathrm{Sv}$ background strain ${ }^{155}$. Smad3 is a component of a heterotrimeric transcription factor functioning downstream of TGF- $\beta$ signaling. Of note, mutations in multiple genes affecting the TGF- $\beta$ pathway have been associated with human CAC and a loss of function mutation in TGF- $\beta$ RII is considered a poor prognostic indicator in CAC. Thus, the Smad3 mouse model of CAC provides a biologically relevant model of the human condition. Inflammation and the subsequent CAC occur as a result of inoculation with $H$. bilis $^{156}$, and, histologically, naïve mice appear completely normal. Based on the 
purported role of $H$. bilis as a provocateur of intestinal immune responses against commensal bacteria ${ }^{79,80}$, the fact that the pathway affected by mutation in $\mathrm{Smad} 3$ is commonly affected in humans diagnosed with CAC, and the phenotypic similarities between $\mathrm{CAC}$ in $\mathrm{Smad}^{-/-}$mice and mucinous adenocarcinoma in humans, this is an ideal model for investigations of diagnostic biomarkers of CAC.

A biomarker is defined as an indicator of a biological state. For example, rather than directly identifying lactate dehydrogenase-elevating virus in mice, the elevation of the lactate dehydrogenase enzyme in peripheral blood provides a relatively accurate biomarker of infection. Biomarkers can conceivably be derived from any biological sample and can take the form of DNA, RNA, protein, or even complex traits such as coat color or odor. The development of molecular, i.e. nucleic acid-based, fecal biomarkers of dysplasia in mouse models of CAC will serve to refine the model and reduce the number of animals needed for adequate statistical power without any invasive sample acquisition. Additionally, diagnosis of CAC in IBD patients is classically made via colonoscopy, a technique fraught with unpleasant aspects and subject to many false negatives. If translated to humans, the development of molecular biomarkers has the potential to increase patient compliance with screening recommendations put forth by the American Gastroenterological Association ${ }^{157}$ and the American College of Gastroenterology, and thus enhance early detection of suspect lesions and patient survival. 


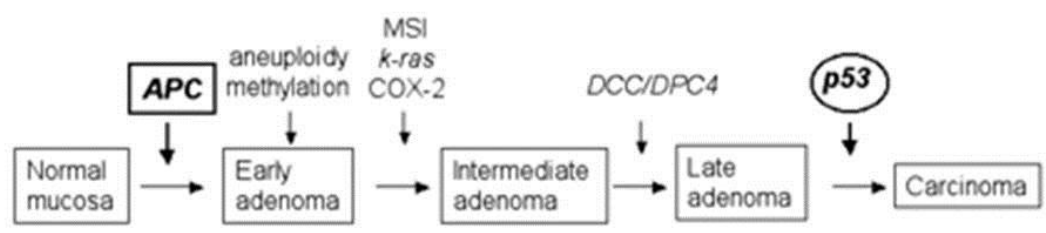

\section{COLITIS-ASSOCIATED COLON CANCER}

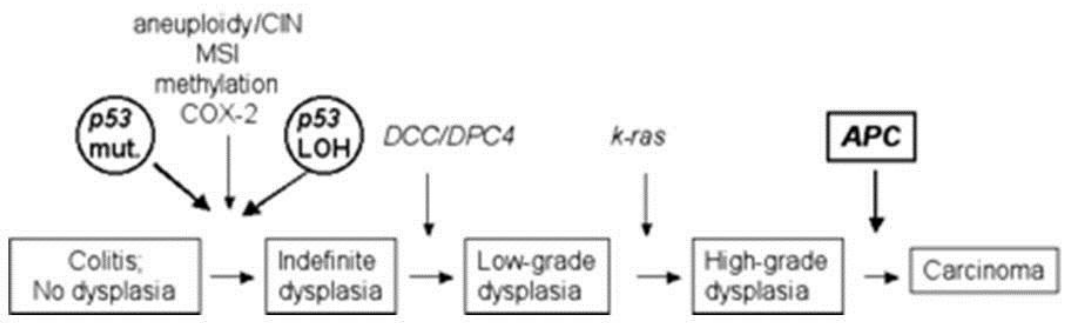

Figure 1.1 Molecular pathogenesis of sporadic colon cancer (top) and colitis-associated colon cancer (bottom). COX-2, cyclooxygenase-2; CIN, chromosomal instability; MSI, microsatellite instability; mut., mutation; LOH, loss of heterozygosity; DCC, deleted in colon cancer; DPC, deleted in pancreatic cancer; APC, adenomatous polyposis coli.

From Itzkowitz SH, Yio X. Inflammation and cancer IV. Colorectal cancer in inflammatory bowel disease: the role of inflammation. Am J Physiol Gastrointest Liver Physiol 2004;287:G7-17. 


\section{CHAPTER 2}

\section{OVERVIEW}

Inflammatory bowel disease (IBD), comprising Crohn's disease (CD) and ulcerative colitis (UC), affects over one million Americans and is now the second most common chronic inflammatory disease after rheumatoid arthritis ${ }^{158}$. The most common manifestations are painful abdominal cramping, recurrent diarrhea, and rectal bleeding, however complications from the chronic inflammation can include obstructions due to fibrosis, fistulae necessitating surgery, nutritional deficiencies, and an increased risk of colorectal cancer correlating with disease duration and the extent of GI involvement ${ }^{159}$.

$\mathrm{CD}$ is characterized by segmental, often transmural, inflammation affecting any region of the GIT from the oral cavity to the anus. The inflammation is thought to be in response to non-pathogenic or commensal intestinal microbes, and while changes in the intestinal microbiota have been identified in IBD patients, no causative etiologic agent has been identified despite numerous attempts ${ }^{74,160-169}$. While the majority of cells in the lesions of CD patients are $\mathrm{T}$ lymphocytes, a component of the adaptive immune system, mounting evidence implicates the response of the innate immune system to the intestinal microbiota as a precursor to the recruitment of $\mathrm{T}$ cells. Specifically, several quantitative trait loci (QTL) have been identified in IBD patients encoding pattern recognition receptors (PRRs) responsible for recognition of bacteria, including but not limited to nucleotidebinding oligomerization domain-containing protein $2(\operatorname{Nod} 2)^{29,31,170,171}$, toll-like receptor (TLR4), and TLR5 ${ }^{172-176}$. Other QTL have implicated the cytokine networks downstream of the recognition of the GI microbiota. For example, mutations in the receptor for IL-23, 
a cytokine produced by DC, may confer resistance ${ }^{177,178}$ or susceptibility to $C D^{179-181}$. Thus, disease susceptibility is, at least partially, determined by the sensing of GI microbiota by the innate immune system.

There are myriad ways of modeling IBD in animals including chemically induced models such as dextran sodium sulfate (DSS)-induced colitis, genetically induced models such as $\mathrm{IL}-10^{-/-}$mice, and microbially induced models such as $H$. hepaticus-infected A/JCr (A/J) and C57BL/6 (B6) mice. In the latter, employed in the present studies, A/J and B6 mice are both colonized equivalently by $H$. hepaticus following gastric gavage, however the $\mathrm{A} / \mathrm{J}$ strain of mouse develops a latent lymphocytic typhlitis following acute perturbations in cytokine expression whereas the B6 strain experiences only transient alterations in cytokine expression and no histological evidence of inflammation. This is an extremely useful model for several reasons. For one, both mouse strains are immunocompetent with no genetic manipulations, mimicking the human condition in which known genetic polymorphisms account for only a portion of disease susceptibility. While knockout and transgenic strains of mice have shed light on the role of specific cytokines in the inflammatory cascade of IBD, the pleiotropic and redundant nature of many cytokines makes it difficult to separate immediate effects of the addition or deletion of a single cytokine from downstream effects. Secondly, the extended period of latency between inoculation and the development of inflammation allows for the investigation of events leading up to disease. Previous studies have revealed several acute changes in cytokine expression in infected mice responsible for setting the stage for the eventual inflammation. Thirdly, disease is induced by inoculation with $H$. hepaticus, providing an ideal microbial trigger for the initiation of disease. The addition of $H$. hepaticus often 
exacerbates other models of IBD ${ }^{119-122}$, though disease is almost always dependent on the presence of background microbiota also. For example, IL-10 $10^{-/}$mice fail to develop intestinal inflammation when mono-associated with $H$. hepaticus, showing that the resident microbiota is essential in the induction of disease even in a host genetically predisposed to intestinal inflammation ${ }^{123}$. Similarly, the introduction of certain Helicobacter spp. into some WT mice with an established microbiota is capable of inducing inflammation, as in $\mathrm{A} / \mathrm{J}$ mice. These contradictions in the pathogenicity of Helicobacter spp. may be explained by findings suggesting that certain Helicobacter spp. act as "provocateurs" of immune responses against commensal microbiota. In the presence of $H$. bilis, gnotobiotic mice generate robust $\operatorname{IgG}$ responses against several members of a defined flora ${ }^{79,80}$ supporting the notion that, while Helicobacter is considered pathogenic in susceptible strains of mice, the inflammation is partially in response to other microbial species as well. As IBD is likely triggered by an event or events that result in abnormal recognition of commensal microbes, the Helicobacterinduced model provides an attractive means of studying this complex process.

Lastly, similar to IBD in humans, the inflammation in $H$. hepaticus-infected A/J mice is largely driven by the innate immune system, as supported by the following findings. Previous studies from our laboratory have examined the expression of several chemokines and cytokines in the ceca of $H$. hepaticus- and sham-inoculated A/J and B6 mice ${ }^{131,182}$. The immune response at 90 days PI in $\mathrm{A} / \mathrm{J}$ mice is $\mathrm{T}_{\mathrm{H}} 1$-mediated, evinced by significant increases in the expression of IL-12/23p 40 , a pro-inflammatory cytokine produced primarily by dendritic cells (DC), and IFN- $\gamma$. Additionally, IL-12/23p40 and several chemokines were found to be significantly upregulated in A/J mice by 4 days PI, 
a time point implicating the innate immune system. Conversely, the immune response of B6 mice was blunted relative to that of $\mathrm{A} / \mathrm{J}$ mice, and chemokine and cytokine levels returned to baseline rapidly or were not elevated compared to uninfected controls, e.g., IL-12/23p 40 . The expression of these mediators is causally linked to the inflammation as antibody neutralization of either IL-12/23p40 or IFN- $\gamma$ significantly decreases cecal lesion scores ${ }^{131}$. As DC are the major intestinal source of IL-12/23p40, its differential expression at 4 days PI in susceptible and resistant mice suggests a role for DC in the pathogenesis of $H$. hepaticus-induced disease.

While the innate immune system comprises several cell types including epithelial cells and natural killer (NK) cells, professional antigen-presenting cells such as dendritic cells (DC) represent a logical avenue of research for several reasons. Firstly, DC are the primary cellular source of IL-12/23p 40 , the defining cytokine in $H$. hepaticus-infected $\mathrm{A} / \mathrm{JCr}$ and $\mathrm{C} 57 \mathrm{BL} / 6$ mice. While the expression of other inflammatory cytokines is transiently elevated following infection of B6 mice, IL-12/23p40 is never differentially expressed between infected and uninfected B6 mice. Additionally, some inflammatory cytokines such as IFN- $\gamma$ and CXCL9 (MIG) are subject to a biphasic elevation in infected $\mathrm{A} / \mathrm{J}$ mice, wherein expression peaks at 4 days post-inoculation (PI), diminishes to baseline levels, and then rises again concurrent with the development of histological evidence of disease. The expression of IL-12/23p40 in A/J mice however rises acutely following infection with $H$. hepaticus and remains elevated indefinitely. Thus, as DC are the main source of IL-12/23p40, it stands to reason that there is either a difference between $\mathrm{A} / \mathrm{J}$ and $\mathrm{B} 6$ mice in the number of total $\mathrm{DC}$ or some subset of $\mathrm{DC}$, or a 
difference in the manner in which the cells in the cecum respond to some $H$. hepaticusmediated stimulus in the cecum.

Further, it is now recognized that there are distinct subsets of DC in the gut. It is vital to discern the role of each subset in exacerbating or ameliorating disease. In mice, conventional intestinal DC are composed of 3 subsets, all of which are CD11 ${ }^{+}$. The three subsets of DC are differentiated by their expression of CD8 $\alpha$ and the integrin subunit $\mathrm{CD} 11 \mathrm{~b}$, resulting in $\mathrm{CD} 11 \mathrm{c}^{+} \mathrm{CD} 8 \alpha^{+} \mathrm{CD} 11 \mathrm{~b}^{-}\left(\mathrm{CD} 8 \alpha^{+}\right), \mathrm{CD} 11 \mathrm{c}^{+} \mathrm{CD} 8 \alpha^{-} \mathrm{CD} 11 \mathrm{~b}^{+}$ $\left(\mathrm{CD} 11 \mathrm{~b}^{+}\right)$, or $\mathrm{CD} 11 \mathrm{c}^{+} \mathrm{CD} 8 \alpha^{-} \mathrm{CD} 11 \mathrm{~b}^{-}$(double negative, $\left.\mathrm{DN}\right) \mathrm{DC}$ in the small intestines and colon. To the author's knowledge, no studies have examined the proportions of these DC subsets in the cecum and, in fact, there are no reports of the number or function of DC in the cecum of any species (based on a search of the National Library of Medicine using the medical subject headings "dendritic cells" and "cecum"). Several studies have demonstrated characteristic responses of each subset in other regions of the gut. CD11 $\mathrm{b}^{+}$ DC are the principal subset responsible for inducing cross-tolerance to endogenous intestinal antigens ${ }^{183}$. Only these cells, and not the $\mathrm{CD} 8 \alpha^{+}$or DN subsets, produce abundant IL-10 upon stimulation with either CD40L or Staphylococcus aureus and IFN$\gamma^{124}$. In turn, $\mathrm{T}$ cells cultured with the $\mathrm{CD} 11 \mathrm{~b}^{+}$subset produce significantly more IL-10 and IL-4 than T cells cultured with CD8 $\alpha^{+}$or DN DC. Additionally, in mice in which the $\mathrm{CD}_{11 \mathrm{~b}^{+}}$subset has been genetically inactivated, oral tolerance is abolished while DC maturation and antigen-specific immune activation appears unaffected ${ }^{184}$. Thus, the $\mathrm{CD}_{11 \mathrm{~b}^{+}}$subset of DC appears to have an immunoregulatory role in intestinal homeostasis, in response to both endogenous and bacterial antigens. The $\mathrm{CD} 8 \alpha^{+}$and DN DC subsets behave quite differently than the $\mathrm{CD} 11 \mathrm{~b}^{+}$subset. In vitro studies indicate that 
both subsets, but particularly CD $8 \alpha^{+}$DC, produce IL-12p70 following stimulation with pathogenic microbes or $\mathrm{CD}_{40} \mathrm{~L}^{124,128}$. Similarly, the DN subset was shown to be responsible for constitutive IL-12/23p40 production in the terminal ileum ${ }^{130}$. While there is evidence that DN DC are also capable of cross-presenting antigen in the intestine ${ }^{185}$, this results in a $T_{H} 1$-biased immune response rather than tolerance as in the case of CD11b ${ }^{+}$DC-mediated cross-presentation ${ }^{183}$. Thus, substantial evidence supports a role for CD8 $\alpha^{+}$and DN DC in the induction of mucosal immune responses.

Considering the above, our overall hypothesis is that the inflammation induced in $H$. hepaticus-infected A/J mice is due to differences in the composition of the intestinal DC populations or the responsiveness of one or more subsets of intestinal DC to some microbial stimulus. Specifically, we hypothesize the disease is due to an excessive number (either absolute or relative) of $\mathrm{CD} 8 \alpha^{+} \mathrm{DC}$ in $\mathrm{A} / \mathrm{J}$ mice, and that cecal cells will produce greater amounts of IL-12/23p40 and other inflammatory cytokines in response to microbial stimulation in $\mathrm{A} / \mathrm{J}$ mice relative to $\mathrm{B} 6$ mice. 


\section{MATERIALS \& METHODS}

Bacteria and Cultivation. A Helicobacter hepaticus isolate (MU-94) was obtained from an endemically infected mouse colony using a previously described culture technique ${ }^{186}$. The isolate was identified as $H$. hepaticus based on ultrastructural morphology, biochemical characteristics, and sequence analysis of the 16s rRNA gene ${ }^{187}$. For inoculation, H. hepaticus cultures were grown in $5 \mathrm{~mL}$ of Brucella broth (Becton Dickinson, Franklin Lakes, NJ) supplemented with 5\% fetal calf serum (Sigma-Aldrich Co., St. Louis, MO) and overlaid on blood agar plates and incubated for 24 to 48 hours at $37{ }^{\circ} \mathrm{C}$ in a microaerobic environment containing $90 \% \mathrm{~N}_{2}, 5 \% \mathrm{H}_{2}$, and $5 \% \mathrm{CO}_{2}$.

Animals. All studies were performed in accordance with the Guide for the Care and Use of Laboratory Animals and were approved by the University of Missouri Institutional Animal Care and Use Committee. Weanling $\mathrm{A} / \mathrm{JCr}$ and $\mathrm{C} 57 \mathrm{BL} / 6 \mathrm{Cr}$ mice were obtained from the Frederick Cancer Research and Development Center, Frederick, MD. Six week old B6.129S6-Rag2 $2^{\text {tmlFwa }}$ mice were purchased from Taconic, Hudson, NY. All mice were confirmed to be free of adventitious viruses, parasites, and pathogenic enteric and respiratory bacteria, including all known murine Helicobacter spp. Three to four week old mice were inoculated with $10^{8} \mathrm{H}$. hepaticus organisms in $0.5 \mathrm{~mL}$ Brucella broth, or an equivalent volume of sterile broth, via gastric gavage. Separate cohorts of mice were used for each study including flow cytometry ( $n=7$ to 11 with 5 to 10 mice pooled per data point) and ex vivo stimulation, ELISA and microbead fluorescent immunoassays ( $n$ $=4$ to 8 groups of 5 mice pooled per data point). Mice were group-housed according to infection status in autoclaved microisolator cages and were provided autoclaved food and 
water. All manipulations and sample collections were performed in a biosafety hood. Mice were euthanized at 0 to 90 days PI via inhaled overdose of $\mathrm{CO}_{2}$.

Tissue Digestion and Cell Isolation. For flow cytometric and ex vivo stimulation experiments, cells were pooled from 5 to 10 mice per data point. Cecal tissue was collected using aseptic technique immediately following euthanasia. Ceca were incised longitudinally and opened such that the mucosal surface was exposed. Cecal tissue was then rinsed vigorously with saline to remove all gross cecal contents. Tissue was then minced with a scalpel blade and placed immediately into a $50 \mathrm{~mL}$ conical tube containing Hank's Balanced Salt Solution (HBSS) supplemented with 5\% fetal bovine serum (FBS), $0.05 \mathrm{mM}$ ethylenediaminetetraacetic acid (EDTA), $0.6 \mathrm{mM}$ 4-(2-hydroxyethyl)-1piperazineethanesulfonic acid (HEPES), $15 \mu \mathrm{g} / \mathrm{mL}$ dithiothreitol (DTT), 100 i.u./mL penicillin, $100 \mu \mathrm{g} / \mathrm{mL}$ streptomycin, and $0.25 \mu \mathrm{g} / \mathrm{mL}$ amphotericin. Tissue was then washed several times and filtered through 100- and 70- $\mu$ m nylon mesh filters to remove remaining bacteria and ingesta. Tissue pieces were then centrifuged at $400 \times \mathrm{g}$ for 10 minutes before aspirating the supernatant entirely and reconstituting in Roswell Park Memorial Institute (RPMI) buffer supplemented with 10\% FBS, $0.6 \mathrm{mM}$ HEPES, and the antimicrobials added to HBSS above. Tissue was then digested in a $50 \mathrm{~mL}$ conical tube containing supplemented RPMI buffer and approximately $1 \mathrm{mg} / \mathrm{mL}$ collagenase $\mathrm{D}$ for two hours at $37^{\circ} \mathrm{C}$ in a shaking incubator $(\mathrm{C} 24 \mathrm{KC}$ refrigerated shaker incubator, New Brunswick Scientific, Edison, NJ). Digested cecal tissue was then filtered through a 70$\mu \mathrm{m}$ nylon mesh filter and the flow-through collected. Cells were then washed repeatedly and filtered through progressively smaller filter pore sizes to remove clumps of epithelial 
cells and remaining pieces of ingesta. Cells in suspension were then counted using a hemocytometer on an inverted light microscope.

Flow Cytometry. All flow cytometry data was collected on a CyAn ADP flow cytometer (Becton Dickinson) and analyzed using Summit 5.2 analysis software. All antibody staining was performed in the presence of Fc block (purified rat $\operatorname{IgG}_{2 b}$ antimouse CD16/CD32 monoclonal antibody, clone 2.4G2)(BD Biosciences) to prevent nonspecific, Fc-mediated adherence of antibodies to mouse Fc receptors. The following were purchased from BD Biosciences: hamster IgG1 anti-mouse CD11c-PE (clone HL3), hamster IgG1 anti-TNP-KLH-PE (clone G235-2356), rat $\operatorname{IgG}_{2 \mathrm{a}}$ anti-mouse CD8 $\alpha$-APC (clone 53-6.7), and rat $\operatorname{IgG}_{2 \mathrm{~b}}$ anti-TNP-KLH-FITC (clone A95-1). The following were purchased from Abcam: rat $\mathrm{IgG}_{2 \mathrm{a}}$ anti-mouse $\mathrm{CD} 11 \mathrm{~b}$ (clone $3 \mathrm{~A} 33$ ) and rat $\mathrm{IgG}_{2 \mathrm{a}}$ antiKLH (clone RTK2758). Briefly, Cells were incubated with $\mathrm{Fc}$ block at $4^{\circ} \mathrm{C}$ for 30 minutes prior to addition of target antibodies. Cells were then incubated an additional hour at $4^{\circ} \mathrm{C}$ before washing the cells twice and resuspending in sterile PBS.

\section{Enzyme-linked Immunsorbent Assay and Microbead Fluorescent Immunoassay.}

Cells isolated from cecal tissue were plated on 24 -well plates at a concentration of $5 \times 10^{5}$ cells in one $\mathrm{mL}$ of supplemented RPMI buffer. Cells were allowed to rest for approximately 18 hours, primed with recombinant mouse IFN- $\gamma$ (Reprokine Ltd., Valley Cottage, NY) at $10 \mathrm{ng} / \mathrm{mL}$ for two hours and then stimulated with live $H$. hepaticus culture (approximately $5 \times 10^{7} \mathrm{CFU} / \mathrm{mL}$ ), $500 \quad \mathrm{ng} / \mathrm{mL}$ purified $\quad$ E. coli $\mathrm{K} 12$ lipopolysaccharide, or $20 \mu \mathrm{L}$ Brucella broth as a negative control. Stimulated cells were then incubated at $37^{\circ} \mathrm{C}$ for 24 hours before collecting the supernatant for analysis. Cytokines and chemokines, including G-CSF, GM-CSF, IFN- $\gamma$, IL-1 $\alpha$, IL-1 $\beta$, IL-2, IL-4, 
IL-5，IL-6，IL-7，IL-8，IL-10，IL-13，IL-15，IL-17，IP-10，KC，MCP-1，MIP-1 $\alpha ，$ RANTES, and TNF- $\alpha$, were measured using either RayBio® Mouse IL-12 (p40/p70) ELISA kit (RayBiotech, Inc. Norcross, GA) or Milliplex® MAP Mouse Cytokine/Chemokine Kit (Millipore Corp., Billerica, MA), following the manufacturer's instructions, using a BioTek® ELx800 plate reader (Biotek, Winooski, VT) or Luminex ( 100 Analyzer (Luminex Corp., Austin, TX) respectively. For ELISA analysis, cells were added to 96 -well plates coated with anti-mouse IL-12 capture antibodies, incubated and washed. Biotinylated secondary antibodies were then added, followed by streptavidin and then TMB for visualization of plate-bound IL-12. For MFI analysis, cell culture supernatants were added to a mixture of unique microbead sets, each set containing specific internal dyes and coated with capture antibodies against one analyte. Following incubation, a set of detection antibodies against each analyte was added, followed by streptavidin-phycoerythrin (SA-PE). Labeled beads were passed in a sheath fluid by a series of lasers and detection filters in order to discern the identity of each microbead (i.e. the specificity of the antibody bound to its surface) as it passed, as well as the relative intensity of SA-PE bound to the microbead, termed the median fluorescent intensity. Both kits provided a mixture of recombinant standards for each analyte measured which were serially diluted and analyzed alongside unknowns to generate a standard curve. For both ELISA and MFI, all samples including standards were analyzed in duplicate.

Generation of Congenic Mice. Female B6.129S6-Rag2 $2^{t m 1 F w a}$ mice, purchased from Taconic, were bred to male wild type $\mathrm{A} / \mathrm{JCr}$ mice to generate heterozygous pups. Male pups were backcrossed to wild type mice of either recipient strain to fix the $Y$ 
chromosome. Each generation thereafter was screened via PCR for the mutated allele. Mice carrying the mutation were then screened via microsatellite analysis to determine the contribution of each parent and select optimal breeders for the subsequent generation. After sufficient backcrosses to yield mice greater than $99 \% \mathrm{~A} / \mathrm{J}$ based on microsatellite analysis, mice were intercrossed to generate homozygous mutants on the $\mathrm{A} / \mathrm{JCr}$ background.

$129 / \mathrm{SvEv}-$ Batf $3^{t m / K m m}$ mice (kindly provided by Dr. Kenneth Murphy from Washington University in St. Louis, MO) were bred to wild type $\mathrm{A} / \mathrm{JCr}$ mice, and male pups heterozygous for the mutated Batf3 were then backcrossed to $\mathrm{A} / \mathrm{JCr}$ mice in order to fix the $\mathrm{Y}$ chromosome. Offspring from this and all subsequent generations were then screened for the mutation via PCR and heterozygous females were backcrossed 6 additional times to $\mathrm{A} / \mathrm{JCr}$ males. Breeders for the last three backcrosses were selected using marker-assisted breeder selection based on genome-wide analysis of a panel of 65 microsatellites representing all chromosomes (3130xl Genetic Analyzer, Applied Biosystems, Grand Island, NY). Briefly, in each generation, multiplexed PCR analysis of 65 microsatellites specific for either $\mathrm{A} / \mathrm{J}$ or $129 / \mathrm{SvEv}$ mice was used to identify the optimal breeders for the subsequent backcross, i.e. those mice carrying the greatest proportion of recipient microsatellite markers. This breeding strategy was used for backcrosses 5 through 7 , followed by a final brother $\times$ sister intercross of heterozygous pups to produce homozygous A/JCr.129-Batf $3^{\text {tm } / K m m}$ mice.

DNA Extraction. DNA was isolated from tail-snips or fecal pellets for genotyping and confirmation of infection status PCRs respectively using DNeasy kit according to manufacturer's instruction. The quantity and quality of DNA was assessed by measuring 
the absorbance at $260 \mathrm{~nm}$ and $280 \mathrm{~nm}$ (Nanodrop-1000 Spectrophotometer, Nanodrop, Wilmington, DE)

Polymerase Chain Reaction. PCRs used for genotyping and confirmation of infection status were performed in an Applied Biosystems 2720 thermal cycler. All PCR products were visualized using a Qiaxcel Genotyping assays included a two-primer assay designed to detect the Neo cassette and a three-primer assay designed to differentiate wild-type, heterozygous, and homozygous knock-out mice. Primer sequences and cycling parameters for the two reactions were kindly provided by Dr. Elizabeth Bryda (University of Missouri) and Dr. Kenneth Murphy (Washington University) respectively. Primers for the two-primer genotyping assay are: CATTCGACCACCAAGCGAAACATC (Neo $\quad$ F) and ATATCACGGGTAGCCAACGCTATG (Neo R). Each $20 \mu \mathrm{L}$ reaction contained $1 \mu \mathrm{L}$ of DNA template, $2 \mu \mathrm{L}$ per reaction of $10 \times$ buffer containing $\mathrm{MgCl}_{2}$ (Roche), $1.25 \mathrm{mM}$ dNTP (Promega), $10 \mu \mathrm{M}$ of Neo F and Neo R primers, 5 U/ $\mu \mathrm{L}$ Taq Fast Start polymerase (Roche), and milliQ deionized water. Thermal cycling consisted of initial denaturation at $95^{\circ} \mathrm{C}$ for 5 minutes followed by 35 cycles of denaturation at $94^{\circ} \mathrm{C}$ for 30 seconds, annealing at $68^{\circ} \mathrm{C}$ for 30 seconds, and extension at $72^{\circ} \mathrm{C}$ for 1 minute. The expected product is $289 \mathrm{bp}$ in length. Primers for the three-primer assay are: TGCTATGCACAAACCACAAACC (p21 F), GTTGTGAGTCGAAACCACGC (p21 R), and CGTTGGCTACCCGTGATATTGC (Neo R). Each $20 \mu \mathrm{L}$ reaction contained 1 $\mu \mathrm{L}$ of DNA template, $4 \mu \mathrm{L}$ per reaction of $5 \times$ Green GoTaq Flexi buffer (Promega), 25 $\mathrm{mM} \mathrm{MgCl}_{2}, 2.5 \mathrm{mM}$ dNTP, $10 \mu \mathrm{M}$ of p21 F, p21 R, and Neo R primers, $5 \mathrm{U} / \mu \mathrm{L}$ GoTaq polymerase (Promega), and milliQ deionized water. Thermal cycling consisted of initial 
denaturation at $95^{\circ} \mathrm{C}$ for 4 minutes followed by 35 cycles of denaturation at $54^{\circ} \mathrm{C}$ for 30 seconds, annealing at $58^{\circ} \mathrm{C}$ for 30 seconds, and extension at $72^{\circ} \mathrm{C}$ for 52 seconds. The expected products are 288 and 750 bp in length for wild-type and knock-out DNA respectively.

\section{STATISTICAL ANALYSES}

All statistical analyses were performed using SigmaPlot 12.0 (SPSS Inc., Chicago, IL).

Dendritic Cell Numbers. Cells isolated from the cecum of naïve, 4 day post-inoculation (PI), and 90 day $\mathrm{PI} \mathrm{A} / \mathrm{JCr}$ and $\mathrm{C} 57 \mathrm{BL} / 6 \mathrm{Cr}$ mice were enumerated using an improved Neubauer hemocytometer on a Nikon model TMS inverted microscope (Nikon Inc., Melville, NY). Multi-channel flow cytometry was used to measure the frequency of cells expressing CD11c, CD8 $\alpha$, and CD11b. The significance of differences in the percentage or absolute number of cells expressing one or more surface markers between strains at each time point was determined using a Student's t-test or Mann Whitney rank sum test for parametric and nonparametric data respectively, with significance being $\mathrm{p} \leq 0.05$.

Ex vivo Cytokine Production. ELISA was used to measure IL-12 (p40 and p70). Microbead fluorescent immunoassays were used to measure G-CSF, GM-CSF, IFN- $\gamma$, IL-1 $\alpha$, IL-1 $\beta$, IL-2, IL-4, IL-5, IL-6, IL-7, IL-8, IL-10, IL-12p70, IL-13, IL-15, IL-17, IP10, KC, MCP-1, MIP-1 $\alpha$, RANTES, and TNF- $\alpha$ production by equivalent numbers of cecal cells isolated from naïve, 4 day post-inoculation (PI), and 90 day PI A/JCr and C57BL/6Cr mice and stimulated ex vivo with live $H$. hepaticus, E. coli (K12) lipopolysaccharide, or culture medium (control). The significance of differences in mean cytokine/chemokine production between strains at each time point and for each stimulus 
was determined using a two-way ANOVA and Dunnett post hoc analysis with significance being $\mathrm{p} \leq 0.05$. 


\section{RESULTS}

\section{Influence of the Adaptive Immune System on H. hepaticus-induced Inflammation.}

To confirm the central role of the innate immune system in both resistance and susceptibility to $H$. hepaticus-induced inflammation, Rag2 (recombinase-activating gene 2)-deficient mice lacking a functional adaptive immune system on both background strains were inoculated with $H$. hepaticus. Similar to WT B6 mice, Rag2 ${ }^{-1-}$ B6 mice develop no inflammation following infection with $H$. hepaticus, suggesting that the adaptive immune system is not required for resistance to $H$. hepaticus-induced cecal inflammation. In Rag2-deficient $\mathrm{A} / \mathrm{J}$ mice however, $H$. hepaticus induces a severe proliferative typhlitis with dysplastic epithelial changes, accompanied by a mononuclear cell infiltrate (figure 2.1). Thus, while the nature of the inflammation differs in the absence of an adaptive immune system, $H$. hepaticus continues to induce mucosal inflammation in $\mathrm{A} / \mathrm{J}$ mice lacking functional $\mathrm{T}$ and $\mathrm{B}$ cells. This, along with the rapidity and nature of the mucosal immune response, strongly suggests that $H$. hepaticus-induced inflammation in $\mathrm{A} / \mathrm{J}$ mice is driven by a cell of the innate immune system.

Number of Dendritic Cells in Cecum of Naïve and Infected A/J and B6 Mice. To evaluate the role of $\mathrm{CD} 8 \alpha^{+} \mathrm{DC}$ in $H$. hepaticus-mediated intestinal inflammation, we first examined the overall cellularity of the cecum of naïve weanling $\mathrm{A} / \mathrm{J}$ and $\mathrm{B} 6$ mice to determine if there are differences between the two strains in the overall cellularity or the total number of DC, as defined by expression of high levels of the pan-DC marker CD11c. Using collagenase-digested pooled cecal tissue, an average of $3.39 \times 10^{5}$ and $2.07 \times 10^{5}$ lamina propria cells were recovered per mouse from naïve A/J and B6 mice respectively $(\mathrm{p}<0.05)$ (figure 2.2A). Similarly, there was a significant difference 
between mouse strains in the percentage of lamina propria cells (LPC) expressing high levels of CD11c (consistent with a DC phenotype), as well as the absolute number of DC in the cecum. In naïve $\mathrm{A} / \mathrm{J}$ mice, an average of $2.29 \%$ of $\mathrm{LPC}$, or $7.77 \times 10^{3}$ cells per mouse, was identified as DC compared to $1.67 \%$ of LPC, or $3.46 \times 10^{3}$ cells per mouse, in B6 mice ( $\mathrm{p}<0.05$ ) (figure 2.2B). Thus, prior to inoculation with H. hepaticus, the ceca of $\mathrm{A} / \mathrm{J}$ mice have greater overall cellularity, and a greater number of $\mathrm{DC}$, professional antigen-presenting cells.

As our hypothesis is that naïve $\mathrm{A} / \mathrm{J}$ mice have either higher total numbers, or proportion, of $\mathrm{CD} 8 \alpha^{+} \mathrm{DC}$ in the cecum than naïve $\mathrm{B} 6$ mice, we next examined the numbers of the various subsets of intestinal DC in both strains using the gating strategy depicted in figure 2.3. Briefly, we first gated on live cells considered $\mathrm{CD} 11 \mathrm{c}^{+}$, and then analyzed the expression of $\mathrm{CD} 8 \alpha$ and $\mathrm{CD} 11 \mathrm{~b}$, resulting in $\mathrm{CD} 8 \alpha^{+} \mathrm{CD} 11 \mathrm{~b}^{-}\left(\mathrm{CD} 8 \alpha^{+} \mathrm{DC}\right), \mathrm{CD} 8 \alpha^{-} \mathrm{CD} 11 \mathrm{~b}^{+}$ $\left(\mathrm{CD} 11 \mathrm{~b}^{+} \mathrm{DC}\right), \mathrm{CD} 8 \alpha^{-} \mathrm{CD} 11 \mathrm{~b}^{-}$(double negative or DN DC), and surprisingly, a previously unreported $\mathrm{CD} 8 \mathrm{a}^{+} \mathrm{CD} 11 \mathrm{~b}^{+}$(double positive or DP DC). While the relative proportions of DC comprising these categories did not differ significantly between A/J and B6 mice, there were significantly greater numbers of $\mathrm{CD} 8 \alpha^{+} \mathrm{DC}$ (mean 364 versus 88 per mouse),

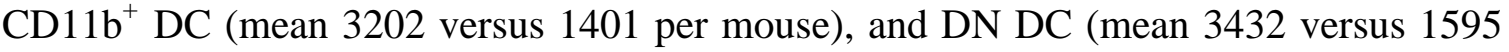
per mouse) in A/J mice relative to B6 mice $(\mathrm{p}<0.05)$ (figure 2.4). There was no difference detected between strains in the number of DP DC. As disease susceptibility may also be due to an imbalance between DC subsets with opposing functions, the ratio of $\mathrm{CD} 8 \alpha^{+} \mathrm{DC}$ to $\mathrm{CD} 11 \mathrm{~b}^{+} \mathrm{DC}$ was also determined. There was no significant difference between $\mathrm{A} / \mathrm{J}$ and $\mathrm{B} 6$ mice in this ratio although there was a trend toward a greater proportion of pro-inflammatory $\mathrm{CD} 8 \alpha^{+} \mathrm{DC}$ relative to $\mathrm{CD} 11 \mathrm{~b}^{+} \mathrm{DC}$ in $\mathrm{A} / \mathrm{J}$ mice $(0.20$ in 
A/J compared to 0.13 in B6 mice). Collectively, these results indicate that there are greater numbers of multiple subsets of cecal DC in naïve A/J mice, including those considered both pro- and anti-inflammatory.

To determine if DC subsets are differentially recruited to the cecal lamina propria of A/J and B6 mice, we next evaluated the cellular composition of the cecum in mice acutely infected with $H$. hepaticus. At four days post-inoculation (4d PI), when expression of IL$12 / 23 \mathrm{p} 40$ is peaking in $\mathrm{A} / \mathrm{J}$ mice, there was again a significantly greater number of cells overall in A/J mice than in B6 mice (figure 2.2A). Similarly, as in naïve mice, the percentage and absolute number of cells expressing CD11c was significantly greater in 4d PI A/J mice when compared to B6 mice $(\mathrm{p}<0.05)$ (figure 2.2B). While the overall cellularity of the cecal lamina propria increased only slightly in both strains of mice between the naïve and 4d PI time points, the number of DC increased substantially in both strains. In $\mathrm{A} / \mathrm{J}$ mice this increase in the number of cecal DC by $4 \mathrm{~d}$ PI achieved significance, going from a median number of $6,455 \mathrm{DC}$ to $17,045 \mathrm{DC}$. Thus, in both mouse strains, DC were recruited to the LP following infection with $H$. hepaticus.

Regarding the subsets of DC in the cecum at $4 \mathrm{~d}$ PI, there were still significant differences between $\mathrm{A} / \mathrm{J}$ and $\mathrm{B} 6$ mice in the numbers of $\mathrm{CD} 8 \alpha^{+}$and $\mathrm{DN} \mathrm{DC}(\mathrm{p}<0.05)$; however, the difference in the number of $\mathrm{CD} 11 \mathrm{~b}^{+} \mathrm{DC}$ was no longer statistically significant (figure 2.5). This is reflected by a comparison within each mouse strain of the numbers of each subset isolated prior to, and 4 days after, inoculation with $H$. hepaticus. In $\mathrm{A} / \mathrm{J}$ mice, there were significant differences between the naïve and $4 \mathrm{~d}$ PI time points in the CD $8 \alpha^{+}$ and DN DC subsets only. In B6 mice, there was no statistically significant difference between the naïve and 4d PI time points in the level of any of the subsets. Collectively, 
these data indicate that while there are higher numbers of both pro- and antiinflammatory subsets of DC in naïve $\mathrm{A} / \mathrm{J}$ mice relative to $\mathrm{B} 6$ mice, the pro-inflammatory subsets are also recruited at a greater rate to the cecal lamina propria in $\mathrm{A} / \mathrm{J}$ mice following experimental infection with $H$. hepaticus.

At 90 days PI (90d PI), the time point at which inflammation has developed in $\mathrm{A} / \mathrm{J}$ mice, there was a significant difference between mouse strains in the overall cellularity of the cecum $(\mathrm{p}<0.05)$ as expected (figure 2.2A). H. hepaticus-infected A/J mice typically have moderate to marked lymphocytic infiltration of the lamina propria by $90 \mathrm{~d} \mathrm{PI}$. Surprisingly however no difference was detected between strains in either the percentage or total number of cells expressing CD11c (figure 2.2B). Additionally, examination of the subsets of DC revealed a trend toward greater numbers of all four subsets of DC in A/J mice relative to B6 mice. However, the differences between strains did not reach statistical significance for any of the DC subsets (figure 2.6). When comparing the relative numbers of each DC subset in 4d PI and 90d PI tissue within each mouse strain, the only difference detected was a significant increase in the number of CD11b $\mathrm{b}^{+} \mathrm{DC}$ in $\mathrm{A} / \mathrm{J}$ mice. Taken together, these data demonstrate that there are disparities in the numbers of both pro- and anti-inflammatory DC subsets in naïve weanling A/J and B6 mice, that pro-inflammatory DC subsets are preferentially recruited to the cecum in A/J mice, and that differences in the numbers of DC subsets are no longer evident by the time at which cells of the adaptive immune system have infiltrated the tissue.

Response of Cecal Dendritic Cells to ex vivo Stimulation. While the results of the above flow cytometric experiments suggest that greater numbers of $\mathrm{CD} 8 \alpha^{+}$and DN DC may play a role in the cecal inflammation induced by $H$. hepaticus in $\mathrm{A} / \mathrm{J}$ mice, there is 
also the possibility that disease susceptibility is due to excessive production of proinflammatory cytokines in A/J mice. To test the hypothesis that cells isolated from the cecum of $\mathrm{A} / \mathrm{J}$ mice will produce greater quantities of inflammatory cytokines and chemokines than equivalent numbers of cells isolated from B6 mice, cells were isolated from the ceca of naïve, $4 \mathrm{~d}$ PI, and $90 \mathrm{~d}$ PI mice, plated at $5 \times 10^{5}$ cells $/ \mathrm{mL}$ and then stimulated ex vivo with $100 \mathrm{ng} / \mathrm{mL}$ recombinant mouse IFN- $\gamma$ followed by $1 \mathrm{ng} / \mathrm{mL} E$. coli LPS or $1 \times 10^{6} \mathrm{CFU} H$. hepaticus. Negative control cultures received $20 \mu \mathrm{L}$ cell culture media. After 24 hours, culture media was then collected for evaluation of cytokine production via MFI or ELISA.

Regarding production of IL-12/23p40, cells isolated from the cecum of naïve $\mathrm{A} / \mathrm{J}$ produced significantly greater amounts than cells from naïve B6, as determined by twoway ANOVA with Dunnett post hoc comparisons. Cell cultures isolated from naïve A/J mice contained significantly greater amounts of IL-12/23p40 at a basal level and when stimulated with $H$. hepaticus or LPS (figure 2.7). There was however no difference within either mouse strain in the level of IL-12/23p40 produced between stimulated and unstimulated cells. Thus, while there is differential production of IL-12/23p40 by cells collected from naïve A/J and B6 mice, this difference is independent of inflammatory stimulus, including LPS and $H$. hepaticus, suggesting that cells from naïve A/J mice are particularly prone to IL-12/23p40 production even in the absence of overt inflammatory stimuli.

No differences were detected between cells isolated from naïve A/J and B6 mice in the production of any other inflammatory cytokines or chemokines examined including IL-6, 
TNF- $\alpha$, and IL-1 $\beta$. Thus, in agreement with the work of Myles et al., IL-12/23p40 appears to be a canonical cytokine in H. hepaticus-induced intestinal inflammation.

To determine if acute infection with $H$. hepaticus affects the response of cells to ex vivo challenge with $H$. hepaticus or LPS, we isolated cells from the cecal lamina propria of $4 \mathrm{~d}$ PI A/J and B6 mice and stimulated as described above. In contrast to the response of cells from naïve mice, there was not a significant difference in the production of IL12/23p40 in cells from 4d PI A/J and B6 mice (figure 2.8A), despite a trend toward overall greater production in $\mathrm{A} / \mathrm{J}$ cells. As before, the level of cytokine production was unaffected by the stimulus added. Of note, cytokine production by cells from both $\mathrm{A} / \mathrm{J}$ and B6 mice was substantially higher than that seen in experiments using cells from naïve mice. Mean IL-12/23p40 production increased from $125.00 \mathrm{ng} / \mu \mathrm{L}$ in naïve $\mathrm{A} / \mathrm{J}$ mice to $369.79 \mathrm{ng} / \mu \mathrm{L}$ in $4 \mathrm{~d} \mathrm{PI} \mathrm{A} / \mathrm{J}$, and from $64.47 \mathrm{ng} / \mu \mathrm{L}$ in naïve $\mathrm{B} 6$ mice to $273.50 \mathrm{ng} / \mu \mathrm{L}$ in $4 \mathrm{~d}$ PI B6 mice. Thus, cecal cells from infected mice of both strains produce substantially greater amounts of IL-12/23p40.

Other inflammatory factors however were produced in significantly greater quantities by cells from 4d PI A/J mice relative to 4d PI B6 mice, including TNF- $\alpha$ (figure 2.8B), CXCL10 (interferon- $\gamma$ induced protein 10, IP-10) (figure 2.8C), and CCL5 (regulated and normal $\mathrm{T}$ cell expressed and secreted, RANTES)(figure 2.8D). When comparing stimuli individually between mouse strains, the production of TNF- $\alpha$ by cells from A/J mice was significantly greater at a basal level, as well as when stimulated with $H$. hepaticus or LPS. Differential production of CXCL10 and CCL5 followed similar patterns and achieved significance in both untreated controls and in cultures stimulated with $H$. hepaticus, suggesting that these chemokines may be critical factors in the 
attraction of other leukocytes in H. hepaticus-mediated inflammation. There was no significant difference detected in the production of any of the other cytokines or chemokines analyzed.

To assess the effect of chronic infection with $H$. hepaticus on cytokine production, we next performed similar experiments using cells isolated from 90d PI A/J and B6 mice. As in the experiments using cells from 4d PI mice, there was no difference in the production of IL-12/23p40 with mean levels of 132.78 and $135.88 \mathrm{ng} / \mu \mathrm{L}$ in $\mathrm{A} / \mathrm{J}$ and $\mathrm{B} 6$ respectively (figure 2.9A). There was also no difference detected in either strain between the stimulated and control cultures. Taken in the context of the above data, cells from A/J mice appear to have a propensity toward exaggerated IL-12/23p40 production, independent of stimuli, early in life, and that following acute and chronic infection with H. hepaticus, the two mouse strains tend to normalize in their production of that cytokine, but respond differentially in their production of other inflammatory mediators.

Similarly, IP-10, shown to be differentially produced in cells collected from 4d PI A/J and B6 mice, tended to normalize in both strains and returned to levels similar to those seen in cells from naïve mice (figure 2.9C).

Production of TNF- $\alpha$ and CCL5 (RANTES) however, was largely undetectable in cell culture supernatants from 90d PI B6 mice and thus maintained the differential expression seen in cells derived from 4d PI mice. Analysis of individual stimuli revealed significant differences in TNF- $\alpha$ production between mouse strains when stimulated with $H$. hepaticus or LPS (figures 2.9B). Analysis of CCL5 production detected significant differences between strains in only $H$. hepaticus-treated cell cultures, despite a trend 
toward greater production in cells from $\mathrm{A} / \mathrm{J}$ mice in all treatment groups (figure 2.9D). Thus TNF- $\alpha$, and potentially CCL5, are produced at greater levels in cells collected from A/J mice acutely or chronically infected with $H$. hepaticus, when stimulated ex vivo with either H. hepaticus or LPS.

Surprisingly, there was also significantly greater production of certain inflammatory factors including CXCL1 (also called Gro $\alpha$ and Neutrophil-activating factor-3) (figure 2.10A), CCL2 (monocyte chemotactic protein-1, MCP-1) (figure 2.10B), and IL-6 in cell cultures of 90d PI B6 mice (figure 2.10C). The production of CXCL1, a chemokine primarily attractant to neutrophils, was significantly greater in 90d PI B6 cell culture in only $H$. hepaticus-treated cultures. Of note, CXCL1 was dramatically elevated in both mouse strains relative to that produced in cell cultures from naïve and $4 \mathrm{~d}$ PI mice. Alternatively, CCL2 production was significantly higher in untreated cultures of 90d PI B6, however no significant difference was detected between strains in the H. hepaticusand LPS-stimulated cells. IL-6 was produced in greater amounts in the untreated and $H$. hepaticus-treated B6 cells, but not the LPS-treated cells.

Collectively, these data confirm that IL-12/23p40 is differentially secreted by cecal cells of mouse strains considered susceptible and resistant to H. hepaticus-mediated intestinal inflammation. The data also demonstrate that, ex vivo, differential production of IL$12 / 23$ p40 exists in naïve weanling mice, but production then tends to normalize between strains thereafter. We have also shown that cell cultures from the ceca of $4 \mathrm{~d}$ PI A/J mice produce significantly greater amounts of other innate inflammatory mediators including TNF- $\alpha$, CXCL10, and CCL5 at a basal level, when compared to cell cultures derived 
from B6 mice. In the case of TNF- $\alpha$ and CCL5, this relatively greater production persists until 90d PI, the time by which inflammation develops.

Taken in the context of which DC subsets are present in the cecum of the two mouse strains prior to and four days after inoculation, it does not appear that the heretofore unidentified $\mathrm{CD} 8 \mathrm{a}^{+} \mathrm{CD} 11 \mathrm{~b}^{+}$(double-positive, DP) are involved in the differential expression of these inflammatory mediators, as this subset of DCs is present in equivalent numbers between strains. While both the $\mathrm{CD} 8 \alpha^{+}$and DN subsets, known to produce IL$12 / 23 p 40$ in conjunction with IL-12p35 and IL-23p19 respectively, are present in significantly greater numbers in $\mathrm{A} / \mathrm{J}$ mice, it is reasonable to believe that the $\mathrm{CD} 8 \alpha^{+}$ subset is responsible for initiating the $\mathrm{T}_{\mathrm{H}} 1$-mediated inflammatory cascade underlying $H$. hepaticus-mediated chronic inflammation.

\section{Generation of A/J.129-Batf $3^{\text {tmIKmm }}$ mice lacking $\mathrm{CDB}^{+}$Dendritic Cells.}

Lastly, we aimed to develop a method for use in future studies of evaluating the physiological significance of $\mathrm{CD} 8 \alpha^{+} \mathrm{DC}$ in $H$. hepaticus-induced inflammation. Elimination of DC in vivo at the outset of these studies was restricted to the CD11c-DTR mouse described by Jung et al. ${ }^{188}$. This technique is less than ideal for our purposes as it would eliminate all DC, not just the CD8 $\alpha^{+}$subset. Recently however, a novel knockout mouse deficient in the transcription factor Batf 3 was created and characterized as being deficient in $\mathrm{CD} 8 \alpha^{+} \mathrm{DC}^{189}$, yet possessing normal numbers of other DC subsets, $\mathrm{CD} 4^{+}$and $\mathrm{CD}^{+} \mathrm{T}$ cells, and B cells. The mouse strain was however generated on a $129 / \mathrm{SvEv}$ background, historically shown to be of intermediate susceptibility to $H$. hepaticusmediated inflammation. Thus, we endeavored to transfer the mutation to the fully 
susceptible $\mathrm{A} / \mathrm{J}$ mouse strain to assess the role in vivo of $\mathrm{CD} 8 \alpha^{+} \mathrm{DC}$, via congenic A/JCr.129- Batf $3^{\text {tmlKmm }}$ mice.

129/SvEv-Batf $3^{\text {tm } I K m m}\left(\right.$ Batf3 $^{-/}$) mice and the genotyping protocol were obtained from the lab of Dr. Kenneth Murphy. Batf $3^{-/}$mice were first bred to wild type (WT) A/J mice to generate heterozygous pups. Male pups were selected for the first backcross to WT A/J mice in order to fix the $\mathrm{Y}$ chromosome in subsequent generations. Pups generated from this and all subsequent backcrosses were first genotyped for the presence of the mutant Batf3 allele to select female breeders for the next mating. Thus, we opted to utilize an established PCR protocol to screen for the presence of the Neo cassette used in the creation of the original Batf3 ${ }^{-/}$mice to differentiate WT from heterozygous pups. For the next four backcrosses, female pups positive the Neo cassette were backcrossed to WT A/J males. Several $\mathrm{NeO}^{+}$potential breeders from the $\mathrm{N} 4$ mating were then genotyped using a panel of 81 microsatellite markers, ranging from 7 to 9 markers per chromosome, capable of differentiating between $\mathrm{A} / \mathrm{J}$ and 129 mice, i.e. marker-assisted congenics. This method, used to select breeders from all subsequent litters, allowed for optimization of breeder selection by selecting pups with A/J markers flanking the mutated Batf3 allele, and discerning those pups possessing the most "A/J-like" genome overall. Following three additional backcrosses, heterozygous pups estimated to be $99 \% \mathrm{~A} / \mathrm{J}$ were intercrossed to generate homozygous $\mathrm{Batf}^{-/-}$mice on an A/J background. To genotype these pups, two PCR assays were performed: the Neo PCR protocol to identify pups harboring the mutated allele, and the protocol supplied by the laboratory donating the mice, to identify mice lacking the WT allele. Using the combined results of the two assays, each performed in duplicate, we were able to identify several male and female 
homozygous Batf ${ }^{-/-}$mice to be used in establishing a breeding colony of A/JCr.129Batf $3^{\text {tmlKmm }}$ mice.

To confirm that these mice maintained the phenotype described by Hildner et al., flow cytometry was used to compare WT A/J, Batf $3^{+/-}$, and Batf $3^{-/-}$mice. Indeed, Batf $3^{-/-}$mice are completely lacking a $\mathrm{CD} 11 \mathrm{c}^{+} \mathrm{CD} 8 \alpha^{+}$population in both the cecum and spleen, indicating that these mice are indeed lacking $\mathrm{CD} 8 \alpha^{+}$DC. Chronic infection studies comparing these and WT A/J mice are underway and will provide direct evidence of the physiological role of $\mathrm{CD} 8 \alpha^{+} \mathrm{DC}$ in $H$. hepaticus-induced typhlitis. 


\section{DISCUSSION}

It has become clear that there are several different types of DC in the mucosal immune system, distinct from the DC found in the periphery, and each with its own phenotype and function. In the gut, these cells are distinguished by the presence or absence of three different surface markers, CD11c, CD11b, and CD8 $\alpha \alpha$, a homodimer formed of two CD8 $\alpha$ subunits. CD11c serves as a universal marker for all conventional DC subsets, while $\mathrm{CD} 11 \mathrm{~b}$ and $\mathrm{CD} 8 \alpha$ are thought to be expressed in a mutually exclusive manner. Additionally, there is a "double-negative" (DN) DC subset expressing neither CD11b nor $\mathrm{CD} 8 \alpha$. While the $\mathrm{CD} 11 \mathrm{~b}^{+}$subset has consistently been detected as the predominant subset in all regions of the GIT, the $\mathrm{CD} 8 \alpha^{+}$subset appears at differing frequencies depending on the region of the GIT examined. To date, no studies have examined the DC subsets present in the mouse cecum.

Several studies have elucidated the function of the various DC subsets in the GIT. While $\mathrm{CD} 8 \alpha^{+}$and DN DC are prone to the production of IL-12 and/or IL-23 ${ }^{190-196}, \mathrm{CD} 11 \mathrm{~b}^{+}$DC express greater levels of immunosuppressive IL-10 and TGF- $\beta^{193,194,197,198}$. While these inclinations have been documented in controlled ex vivo environments, the characteristic function of each subset in vivo may result from their location in the LP, as each DC subset resides in a specific subanatomic location within the PP. For example, the $\mathrm{CD}_{11 \mathrm{~b}^{+}}$subset is situated directly beneath the follicle-associated epithelium (FAE) and in the subepithelial dome region, putting these cells in closer proximity to the commensal bacteria within the lumen of the GIT. Notably, this subset is also in close proximity to the epithelium which is integral in the suppression of DC responses to certain inflammatory stimuli via thymic stromal lymphopoietin and other factors ${ }^{199-202}$. 
Alternatively, $\mathrm{CD} 8 \alpha^{+} \mathrm{DC}$ reside primarily in the T cell-rich interfollicular regions of PP. Thus, the reported production of IL-12 by this DC subset is consistent with the dominant $\mathrm{T}_{\mathrm{H}} 1$ profile seen in physiological inflammation ${ }^{203}$, and the response to pathogenic microbes may reflect the quantity, rather than quality, of cytokine produced. Additionally, attempts to convert one subset into another using various cytokines and growth factors have proven universally unsuccessful suggesting that the $\mathrm{CD} 8 \alpha^{+}, \mathrm{CD} 11 \mathrm{~b}^{+}$, and DN DC are terminally differentiated subsets and not just developmental stages of $\mathrm{DC}^{197,204}$.

Predictably, the DC subsets home to their individual niches via chemokine gradients and the differential expression of specific chemokine receptors. All three subsets are attracted to PP and the LP by CCL19 and CCL21 via CCR7 expressed on all CD11c ${ }^{+}$ cells. DN DC can also respond to CCL20 (via CCR6), and CD11b ${ }^{+}$DC constitutively respond to CCL20 and CCL9 (via CCR6 and CCR1 respectively). Of note, Crohn's disease (CD) patients express significantly higher levels of CCL19, CCL21, and CCR7 than healthy individuals ${ }^{205}$, and the increase in expression correlates with higher numbers of mature $\mathrm{DC}^{206}$, implicating increased DC trafficking in the pathogenesis of $\mathrm{IBD}^{205}$. Similarly, expression of CCL20, produced by the FAE, is significantly greater in CD patients, compared to healthy controls and patients with $\mathrm{UC}^{207}$. The ability to manipulate these and other trafficking signals remains a relatively under-utilized avenue of therapeutic intervention.

Regarding the frequencies of each subset in tissue, Jang et al. found that in the small intestines, DC constitute approximately 10-15\% of lamina propria leukocytes and are

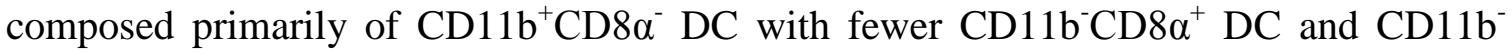


$\mathrm{CD} 8 \alpha^{-}$(double negative; DN) $\mathrm{DC}^{208}$. Within the Peyer's patches (PP) of the small intestines, the three subsets are closer to each other in number although the $\mathrm{CD} 8 \alpha^{+}$and DN population is still the minority ${ }^{124}$.

The proportions of each subset described above remain relatively unchanged throughout the jejunem ${ }^{130}$ but changes in the ileum, wherein bacterial densities increase dramatically. Here, $\mathrm{CD} 8 \alpha^{+} \mathrm{DC}$ are greatly reduced and the double negative population presides as the dominant subset of DC. Of note, this is also the principal region of the GIT wherein DC extend dendrites between epithelial cells in a $\mathrm{CX}_{3} \mathrm{CR} 1$-dependent manner to directly sample the bacteria present within the lumen. Despite this fact however, PP appear to be the predominant site of antigenic recognition and induction of tolerance or immunity by the adaptive immune system ${ }^{209}$. In the colon, the $\mathrm{CD} 11 \mathrm{~b}^{+} \mathrm{DC}$ is again the predominant subset constituting around 50\% of DC, with approximately 30\% DN DC, and $20 \%$ $\mathrm{CD} 8 \alpha^{+} \mathrm{DC}^{204}$. To date, no studies have examined which DC populations exist in the cecum, a region of the GIT specialized for absorption of electrolytes and the digestion of high fiber material, containing prominent lymphoid tissue, particularly in the apical portion, and a specialized and distinct microbial population ${ }^{210}$. Additionally, the few studies that have associated microbially induced inflammation with the function of intestinal DC subsets utilized Staphylococcus aureus, a dermal pathogen, as the inflammatory stimulus ${ }^{124}$.

In the present study, we employed a model of inflammatory bowel disease (IBD) in which cecal inflammation is induced via experimental infection with the opportunistic pathogen $H$. hepaticus. Not all mouse strains are susceptible to $H$. hepaticus-induced inflammation; while both strains are readily colonized, inbred $\mathrm{A} / \mathrm{JCr}(\mathrm{A} / \mathrm{J})$ mice are 
susceptible to inflammation, while C57BL/6 (B6) serve as a resistant control. Following inoculation with $H$. hepaticus, $\mathrm{A} / \mathrm{J}$ mice experience acute fluctuations in the expression of several inflammatory cytokines and chemokines but histologically visible inflammation does not develop until ninety days post-inoculation (PI), presumably due to two factors. First, H. hepaticus is not an overt pathogen in that mono-association with the microbe does not cause disease in any strain tested, and only some strains are susceptible to $H$. hepaticus-mediated enteric inflammation. Second, H. hepaticus has been shown to cause intestinal inflammation by inducing host immune responses against non-pathogenic commensal microbes, likely resulting from a $H$. hepaticus-mediated barrier defect. Thus, collectively, $H$. hepaticus is thought to behave as a provocateur, providing an ideal model of IBD in which aberrant immune responses are directed against commensal microbes.

In the cecum of naïve susceptible $\mathrm{A} / \mathrm{J}$ mice, we detected significantly greater numbers of DC, both as a percentage of cells overall and in absolute number, relative to diseaseresistant B6 mice. Regarding the different subsets of DC described in other regions of the GIT, we hypothesized that the cecum of $\mathrm{A} / \mathrm{J}$ mice would harbor greater proportions of $\mathrm{CD} 8 \alpha^{+}$, or perhaps DN DC, the populations considered to be the main sources of IL$12 / 23 \mathrm{p} 40$. We considered $\mathrm{CD} 8 \alpha^{+} \mathrm{DC}$ to be more likely as the DN population in the ileum was shown to produce primarily IL-12/23p40 in conjunction with IL-23p19 130 whereas the IL-12/23p40 detected in A/J mice skews the immune response toward a $\mathrm{T}_{\mathrm{H}} 1$ profile as determined by a comprehensive cytokine pathway analysis ${ }^{131}$. In naïve $\mathrm{A} / \mathrm{J}$ mice, we detected significantly higher numbers of CD $8 \alpha^{+}$and DN DC and, surprisingly, also CD11b ${ }^{+}$DC, shown to produce abundant IL-10 when stimulated ${ }^{124,211}$. Thus, there are greater numbers of all previously characterized DC subsets in the cecum of naïve A/J 
mice. Of note, we also identified a novel and distinct subset of DC in the cecum of both mouse strains expressing both CD8 $\alpha$ and CD11b (double positive; DP DC). These cells constituted between 10 and $15 \%$ of the $\mathrm{CD}_{11 \mathrm{c}^{+}}$cells and expressed both markers at levels equivalent to the single-positive subsets, however their numbers did not differ significantly between strains and the function of these cells is unclear.

Myles et al. determined that, following inoculation with $H$. hepaticus, a peak in inflammatory cytokine transcription occurs at approximately four days (4d) PI. The expression of these cytokines then either gradually wanes and increases again coincident with the development of inflammation, or remains elevated throughout the first 90 days $\mathrm{PI}^{131}$. In particular, IL-12/23p40 is significantly elevated in $4 \mathrm{~d} \mathrm{PI} A / \mathrm{J}$ mice relative to uninfected controls, and remains elevated until inflammation occurs. Additionally, IL-12 is causative in this model as antibody neutralization of IL-12 abrogates disease completely. Thus, as DC are recognized as the primary source of IL-12, we next sought to determine if one subset of DC was differentially recruited to the cecum of $\mathrm{A} / \mathrm{J}$ mice at 4d PI. Our results demonstrate that both $\mathrm{CD} 8 \alpha^{+}$and DN DC are again present in significantly greater numbers in the ceca of $\mathrm{A} / \mathrm{J}$ mice, relative to $\mathrm{B} 6 \mathrm{mice}$, at $4 \mathrm{~d}$ PI. There was however no longer a significant difference between strains in the number of the immunosuppressive $\mathrm{CD}_{1} 1 \mathrm{~b}^{+}$subset. Comparing the numbers of each subset within each mouse strain at the different time points, it is apparent that $\mathrm{CD} 8 \alpha^{+}$and $\mathrm{DN} \mathrm{DC}$ are recruited to the cecum of $\mathrm{A} / \mathrm{J}$ mice following infection with $H$. hepaticus; of note, $\mathrm{CD} 8 \alpha^{+}$ DC are increased three-fold in 4d PI A/J mice. Comparison of naïve and 4d PI numbers of each subset within each mouse strain revealed that only CD8 $\alpha^{+}$and DN DC are present in significantly greater numbers in $\mathrm{A} / \mathrm{J}$ mice and none of the subsets is present at a 
significantly greater frequency in B6 mice. Overall increases of DC have been reported in the colon of SCID mice with adoptive transfer of $\mathrm{CD}^{2} 5 \mathrm{RB}^{\text {high }} \mathrm{T}$ cells ${ }^{212}$ or intraperitoneal injection of $\mathrm{T}$ cell blasts ${ }^{125}$. Interestingly, Karlis et al. determined that very few of those $\mathrm{DC}$ expressed $\mathrm{CD} 8 \alpha^{+}$prior to disease induction, and that the number of that subset did not change appreciably during inflammation. There are however many variables between that and the present study including mouse strain, the microbiota, and the presence of an adaptive immune system.

By 90d PI, when inflammation is fully developed in A/J mice, there were no longer any differences between strains in the number of any DC subset. It is noteworthy however that the frequency of $\mathrm{CD} 11 \mathrm{~b}^{+} \mathrm{DC}$ had increased dramatically from the $4 \mathrm{~d}$ PI samples in B6 mice, while their numbers at $4 \mathrm{~d}$ and $90 \mathrm{~d}$ PI were roughly equivalent in $\mathrm{A} / \mathrm{J}$ mice. This was unexpected as Myles et al. showed that IL-10 is significantly elevated in infected versus uninfected $\mathrm{A} / \mathrm{J}$ mice at $90 \mathrm{~d} \mathrm{PI}$, and is expressed at much higher levels than in 90d PI B6 mice ${ }^{131}$. It must be remembered however that IL-10 is also produced by regulatory $\mathrm{T}$ cells and some B cells.

Thus, our data support the hypothesis that susceptibility to $H$. hepaticus-induced cecal inflammation in $\mathrm{A} / \mathrm{J}$ mice is due, at least in part, to greater numbers of IL-12/23p40producing DC subsets at the site of inflammation. Our findings also document that the increased presence of these inflammatory subsets exists only during the early stages of disease.

While differences in the frequency of the various DC subsets may explain the disease susceptibility of A/J mice, there is also the possibility that the DC in A/J may have an 
increased capacity to produce inflammatory cytokines and chemokines, when compared to equivalent numbers of DC from B6 mice. To test this, we isolated all mononuclear cells from the cecum of naïve, 4d PI, and 90d PI A/J and B6 mice, and treated them with either live $H$. hepaticus at a density of approximately $10 \mathrm{cfu}$ per cell, or E. coli LPS. Previous studies by MacPherson et al. showed that DC isolated from the mesenteric lymph nodes draining the gut are more activated and mature than DC present within the LP and PP of the gut ${ }^{209}$. To increase the possibility that observed cytokine production was a result of the ex vivo stimulus, we isolated cells from the cecal LP and associated lymphoid follicles to obtain a greater proportion of cells that had not yet been activated.

Bulk cells were used for two specific reasons. Considering the rarity of DC in intestinal tissue of immunocompetent mice, detected at a frequency of $1.6 \%$ and approximately $2 \%$ in the small intestines and colon respectively ${ }^{125}, 212,213$, it would require excessive numbers of mice to obtain adequate cell numbers for ex vivo stimulation. Secondly, the inclusion of other cell types present in a physiological setting allows for a more realistic estimate of the capacity of DCs to produce inflammatory mediators, many of which depend on paracrine signals for production. Admittedly, epithelial cells are lacking from the ex vivo cultures used in the present studies, the primary reason being the exclusion of epithelial organoids (cell aggregates) due to the necessary filtration of tissue digests in the isolation of cecal cells. Additionally, anoikis, or epithelial cell necrosis in the absence of stromal factors, is problematic in primary cell cultures.

It should be noted that the production of inflammatory cytokines and chemokines in $H$. hepaticus-infected A/J and B6 mice was already surveyed by Myles et al. using RT-PCR to analyze gene transcription ${ }^{131,182}$. There are however multiple mechanisms by which 
the actual level of protein produced is regulated, such as RNA editing via Adenosine Deaminase Acting on RNA (ADAR) enzymes ${ }^{214}$, and miRNA which affect the stability of gene transcripts and thus the amount of functional protein produced ${ }^{215}$. Additionally, Myles et al. primarily compared cytokine production between $H$. hepaticus-infected and uninfected mice of each strain at several time points rather than between mouse strains as in the present study. Hence, our goal was to evaluate the capacity of cells derived from naïve and $H$. hepaticus-infected to produce functional inflammatory cytokines and chemokines at the protein level.

Using cells isolated from naïve weanling mice, there was a significant difference between mouse strains in the production of IL-12/23p40 both at a basal level and in cells treated with $H$. hepaticus or LPS. There was however no difference between stimulated and unstimulated cells in the amount of IL-12/23p40 produced. Surprisingly, no differences were detected between strains in the production of any of the other 22 cytokines or chemokines analyzed, including IL-17, TNF- $\alpha$, or IL-6. While H. hepaticus-induced inflammation in $\mathrm{A} / \mathrm{J}$ mice is a $\mathrm{T}_{\mathrm{H}} 1$-mediated process, we expected to see differences in chemokines responsible for the attraction of $\mathrm{T}$ cells, particularly those transcribed at increased levels in H. hepaticus-infected A/J mice such as CXCL10.

This cytokine profile changed when using cells isolated from 4d PI A/J and B6 mice. Despite a trend toward greater IL-12/23p40 production in H. hepaticus- and LPS-treated cells from $\mathrm{A} / \mathrm{J}$ mice when compared to cells from B6 mice, there was no significant difference between strains. Additionally, the abundant IL-12 production seen in cells from 4d PI B6 mice is in stark contrast to previous data showing a complete lack of increased IL-12/23p40 transcription in H. hepaticus-infected B6 mice ${ }^{131}$, presumably a 
result of the tolerizing effects of epithelial cells ${ }^{200-202}$ present in the experiments performed in live animals. In ex vivo stimulation, epithelial cells were removed during the tissue digestion process and only individual mononuclear cells were plated, allowing for more robust inflammatory responses. Alternatively, significantly greater production of TNF- $\alpha$, CXCL10 (IP-10), and CCL5 (RANTES) was now detected in cultures of cells isolated from $4 \mathrm{~d}$ PI A/J mice. The increased production of TNF- $\alpha$ was unexpected as increased transcription in tissue was not noted in prior studies ${ }^{131,182}$. It is interesting however in light of the fact that TNF- $\alpha$ is elevated in both the serum ${ }^{216}$ and stool $^{217}$ of IBD patients, likely due to a polymorphism in the TNF promoter ${ }^{218}$. Additionally, neutralizing antibodies specific for TNF- $\alpha$ including infliximab, adalimumab, and certolizumab pegol have shown great promise in the treatment of different forms of IBD. The increased production of TNF- $\alpha$ by cells of $4 \mathrm{~d}$ PI A/J mice underscores the utility of this model in the study of the human condition.

It is worth noting that the two chemokines produced in greater amounts by cells of $4 \mathrm{~d} P \mathrm{PI}$ A/J mice, CXCL10 (IP-10) and CCL5 (RANTES), are traditionally thought of as being produced, or induced, by cells of the adaptive immune system. Recent work however has demonstrated that both of these chemokines can accumulate very rapidly due to activation of NK and NKT cells ${ }^{219,220}$. This is in accord with anecdotal evidence of a role for NK cells in $H$. hepaticus-mediated IFN- $\gamma$ production by as early as 4 days $\mathrm{PI}^{131}$. While there is some evidence of a role for NK and NKT cells in the pathogenesis of $\mathrm{IBD}^{221,222}$, their contribution to $H$. hepaticus-induced inflammation is unknown. The CXCL10/CXCR3 axis has previously been implicated in $H$. hepaticus-mediated inflammation in $\mathrm{A} / \mathrm{J}$ mice, as well as other models of $\mathrm{IBD}^{223}$ and the human condition ${ }^{224}$. 
Interestingly, TNF- $\alpha$ is a potent inducer of CXCL10, providing additional evidence that this pathway is involved in this model of IBD and a potential avenue for therapeutic exploitation in humans.

Using cells isolated from 90d PI mice, a pattern similar to that of 4d PI mice was observed; there was again no difference in the production of IL-12/23p40 while cell cultures derived from A/J mice contained significantly more TNF- $\alpha$ and CCL5. As in the experiments using 4d PI mice, there was no significant difference between stimulated and untreated cells, indicating that while cells from $H$. hepaticus-infected mice have an increased capacity for production of these inflammatory mediators, it is not directly associated with stimulation by H. hepaticus or E. coli LPS.

Surprisingly, we found that cells isolated from 90d PI B6 mice produced greater amounts of IL-6 and the chemokines CXCL1 (KC) and CCL2 (MCP-1). IL-6 is an innate inflammatory cytokine typically produced in concert with TNF- $\alpha$, making its relatively greater production in cells from B6 mice that much more unexpected. While IL-6 is traditionally considered pro-inflammatory, there are reports that in some instances it exerts anti-inflammatory effects due to an ability to induce circulating IL-1 receptor antagonist and soluble TNF receptor $\mathrm{p} 55^{225}$. Additionally, IL-6 and TGF- $\beta$ function synergistically to induce the differentiation of $T_{H} 17$ cells, which may, in turn, inhibit $T_{H} 1$ development via down-regulation of IL- $12 R \beta 2^{226}$. It is also noteworthy that the chemokines produced at greater levels in B6 cultures, CXCL1 and CCL2, are chemotactic for neutrophils and monocytes respectively, whereas those chemokines found in greater amounts in A/J cultures, CXCL10 and CCL5, are primarily chemotactic for $\mathrm{T}$ cells. Thus, those chemokines the production of which is favored in $\mathrm{A} / \mathrm{J}$ mice are 
more logical factors in mediating the lymphocytic infiltration seen histologically in this model of IBD and in clinical IBD.

Surprisingly, IL-10 was undetectable at any time point in either strain, in contrast to Myles' data. This, and other data discordant with earlier studies of in gene transcription in tissue, are presumably due to differences in the experimental design, as well as differences in the method of cytokine quantitation.

Collectively, our data support the hypotheses that there are differences between A/J and B6 mice in both the number of inflammatory DC subsets and their capacity for the production of specific inflammatory mediators including IL-12/23p40, TNF- $\alpha$, CXCL10, and CCL5.

\section{Development of A/JCr.129- Batf $3^{\text {tmIKmm }}$ mice}

While the above data correlate nicely with the observed disease susceptibility of $\mathrm{A} / \mathrm{J}$ mice, the true physiological significance of $\mathrm{CD} 8 \alpha^{+} \mathrm{DC}$ will need to be verified using an in vivo model. With that aim in mind, we developed the congenic the A/JCr.129Batf $^{\text {tmlKmm }}$ mouse strain lacking $\mathrm{CD} 8 \alpha^{+}$DC. The original knock-out mouse on a $129 / \mathrm{SvEv}$ background lacked only the $\mathrm{CD} 8 \alpha^{+} \mathrm{DC}$ subset and retained all other DC subsets and leukocytes examined. The congenic strain on an A/J background is similar in phenotype and we hypothesize that disease will subsequently be reduced or greatly abrogated. Studies are ongoing to confirm the in vivo relevance of these cells to chronic enteric inflammatory disease. 


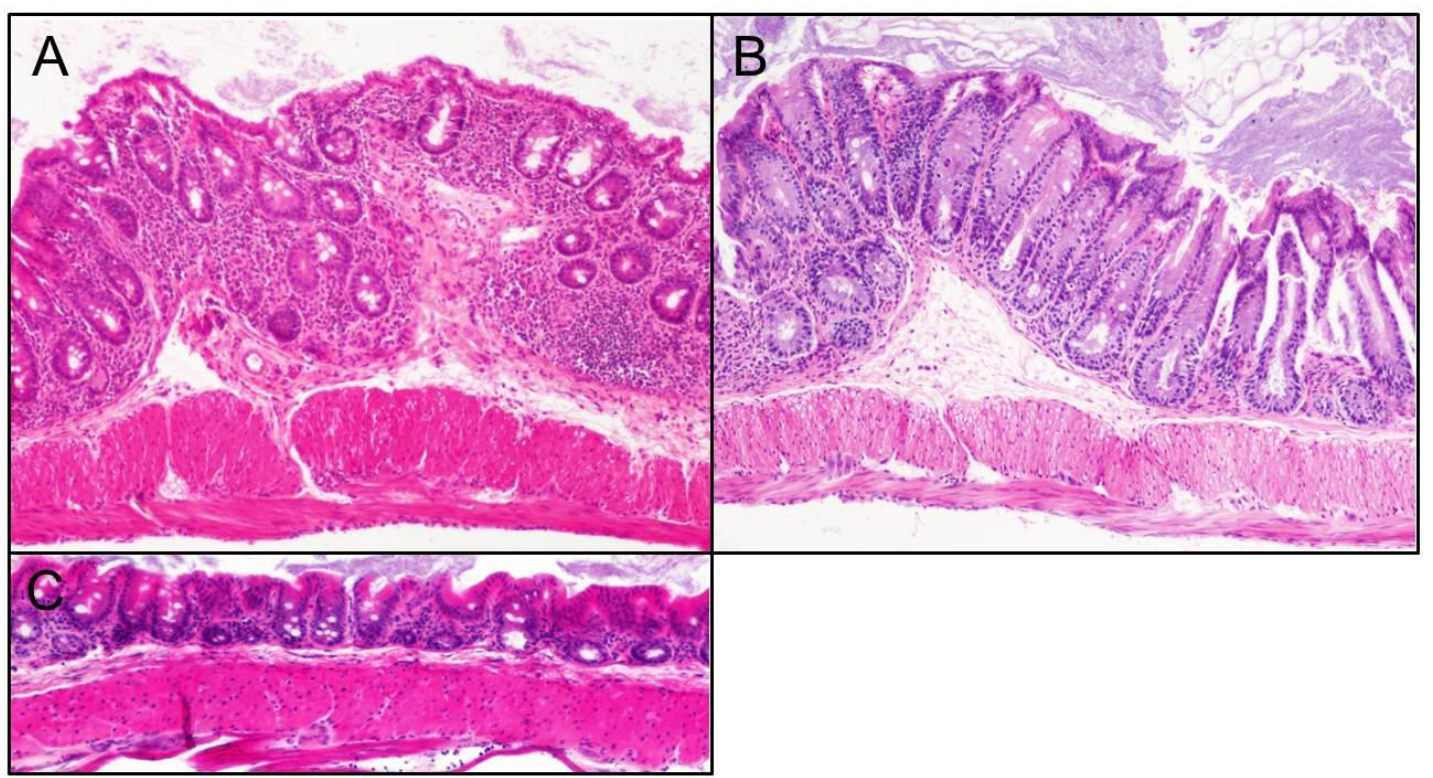

Figure 2.1 Representative images demonstrating the typical histological appearance of cecal sections from $H$. hepaticus-infected wild type A/JCr (A), A/JCr.B6-Rag2 (B), and wild type C57BL/6 (C) mice at 90 days post-inoculation. Hematoxylin and eosin stain at $200 \times$ magnification. 


\section{A Total Cecal Lamina Propria Cells}

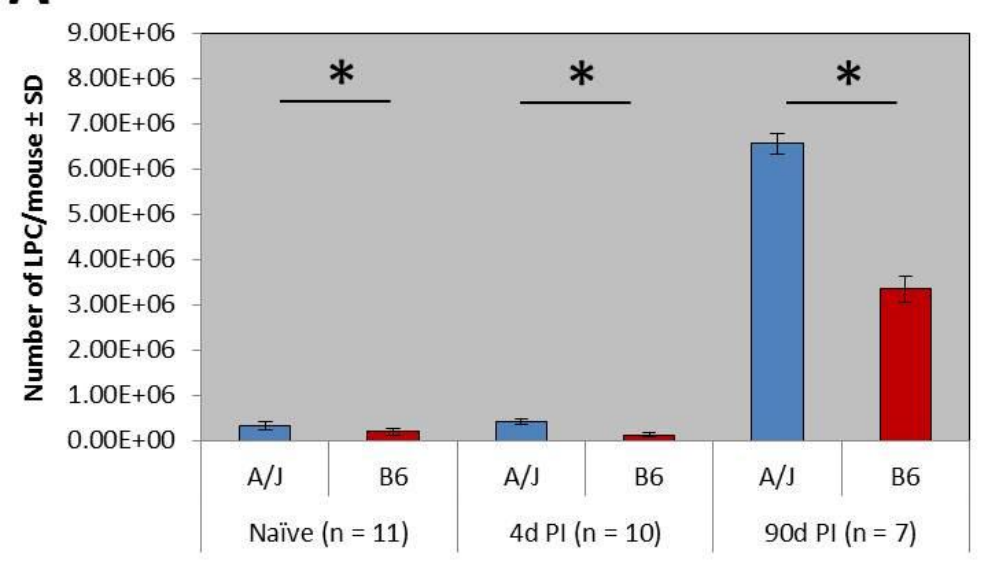

B

\section{Total Cecal Dendritic Cells}

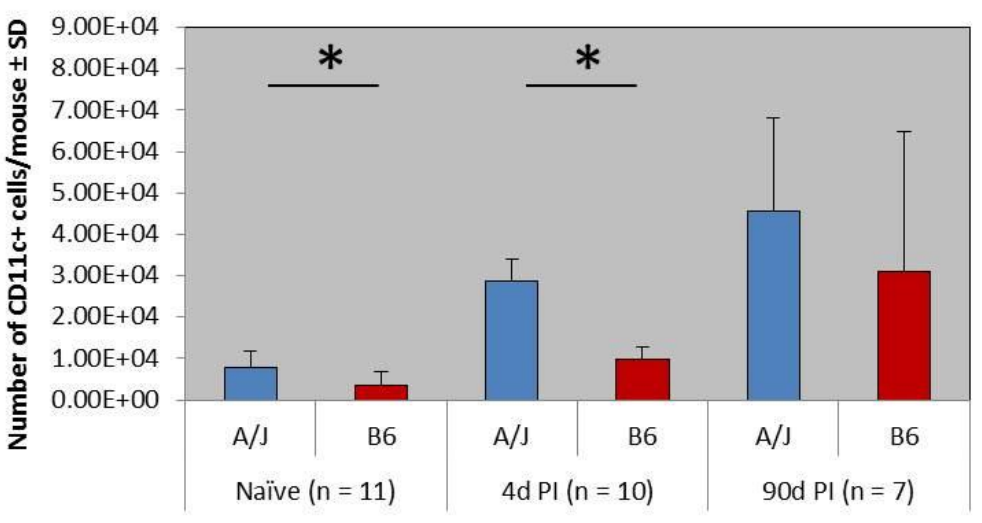

Figure 2.2 Total number of cecal lamina propria (A) and dendritic (B) cells isolated per mouse from naïve four-week old, 4 day postinoculation (4d PI), and 90d PI female A/J and C57BL/6 (B6) mice \pm $\mathrm{SD}(n=7-11)$. Asterisks represent significant difference $(\mathrm{p}<0.05)$ as determined by Mann-Whitney rank sum test. 

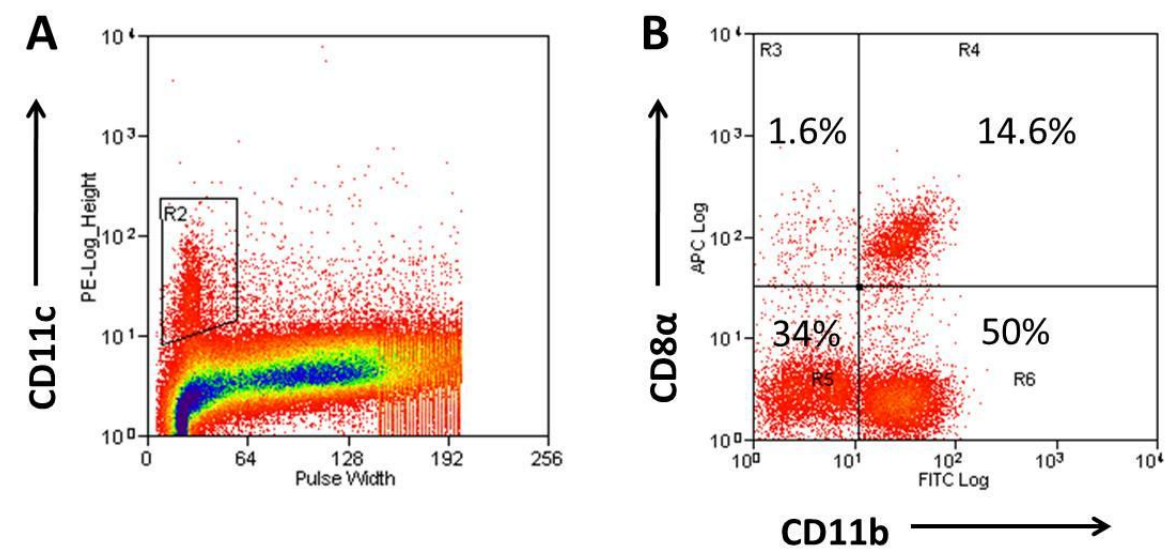

Figure 2.3 Representative image of flow cytometric gating strategy used for identification of cecal dendritic cells (DC) and the four constituent subsets. $2.3 \mathrm{~A}$ shows the gate delineating live $C D 11 \mathrm{c}^{+}$cells (R2); within R2, 2.3B shows the gates delineating $C D 8 \alpha^{+} C D 11 b^{-} D C$ (R3), CD8 $\alpha^{+} C D 11 b^{+} D C$ (R4), CD8 $\alpha^{-C D} 11 b^{-}$DC (R5), and CD8 $\alpha^{-}$ CD11b+ DC (R6). 


\section{Subsets in naïve A/J and B6 Mice}

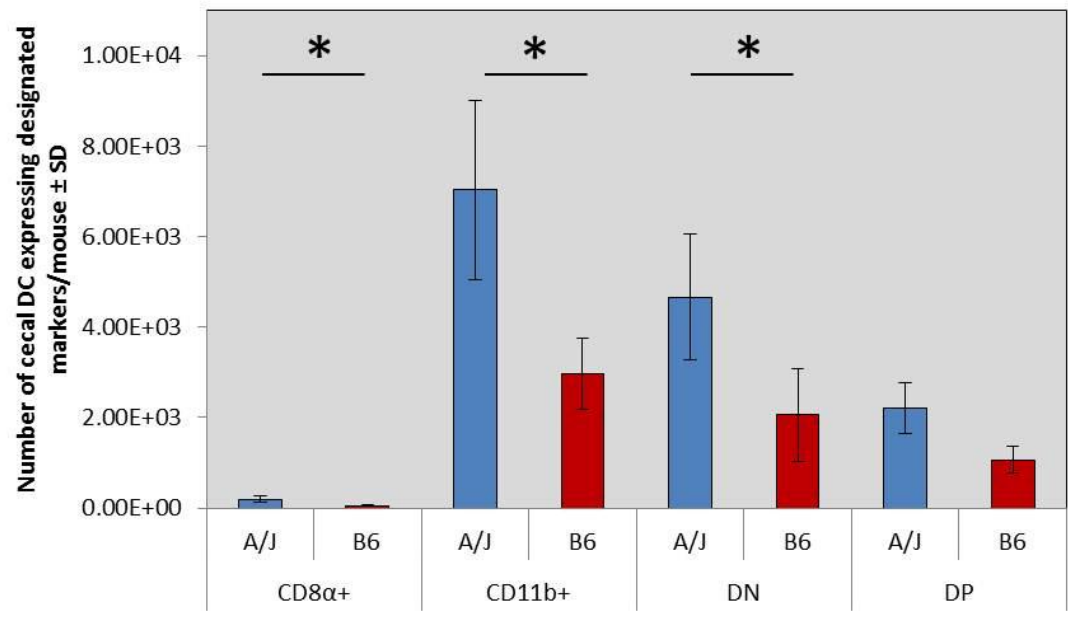

Figure 2.4 Number of cecal dendritic cells isolated per mouse from naïve four-week old female $\mathrm{A} / \mathrm{J}$ and $\mathrm{C} 57 \mathrm{BL} / 6$ (B6) mice determined via flow cytometry to be $C D 8 \alpha^{+} C D 11 b^{-}\left(C D 8 \alpha^{+}\right)$, $\mathrm{CD}^{2} \alpha^{-} \mathrm{CD}_{11} \mathrm{~b}^{+}\left(\mathrm{CD} 11 \mathrm{~b}^{+}\right), \mathrm{CD}^{-} \mathrm{a}^{-} \mathrm{CD} 11 \mathrm{~b}^{-}(\mathrm{DN})$, or CD8 $\alpha^{+} \mathrm{CD} 11 \mathrm{~b}^{+}$ $(D P) \pm S D(n=8-11)$. Asterisks represent significant difference $(p<0.05)$ as determined by Mann-Whitney rank sum test. 


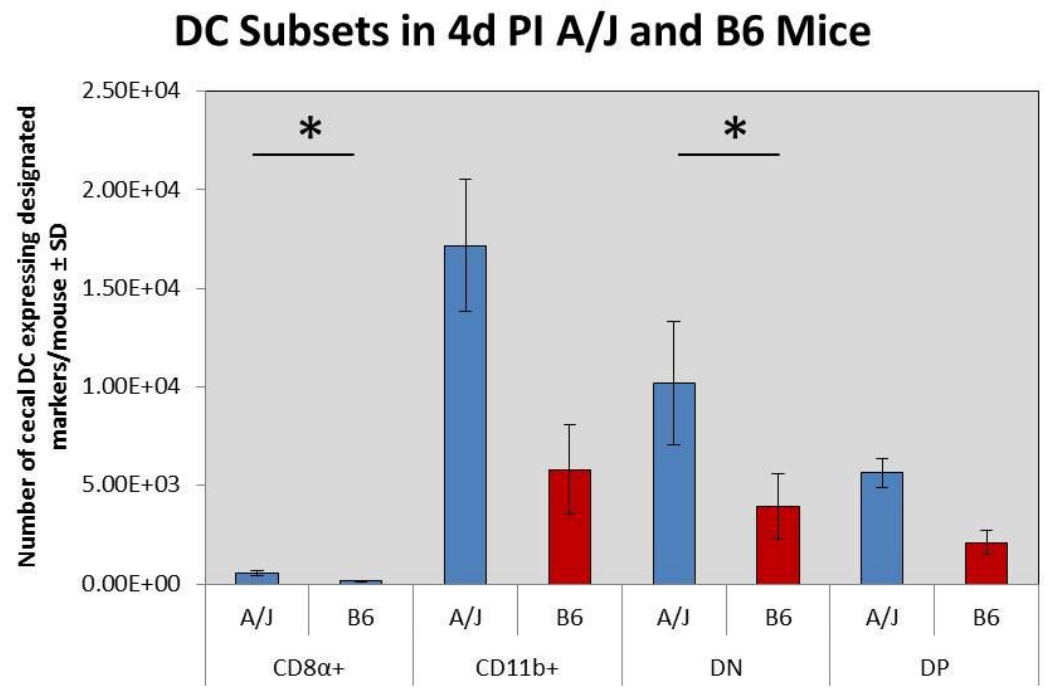

Figure 2.5 Number of cecal dendritic cells isolated per mouse from 4 day post-inoculation (4d PI) female $\mathrm{A} / \mathrm{J}$ and $\mathrm{C} 57 \mathrm{BL} / 6$ (B6) mice determined via flow cytometry to be CD8 $\alpha^{+} C D 11 b^{-}$ $\left(C D 8 \alpha^{+}\right), C D 8 \alpha^{-C D} 11 b^{+}\left(C D 11 b^{+}\right), C D 8 a^{-} C D 11 b^{-}(D N)$, or $\mathrm{CD} 8 \alpha^{+} \mathrm{CD} 11 \mathrm{~b}^{+}(\mathrm{DP}) \pm \mathrm{SD}(n=8-11)$. Asterisks represent significant difference $(p<0.05)$ as determined by MannWhitney rank sum test. 


\section{Subsets in 90d PI A/J and B6 Mice}

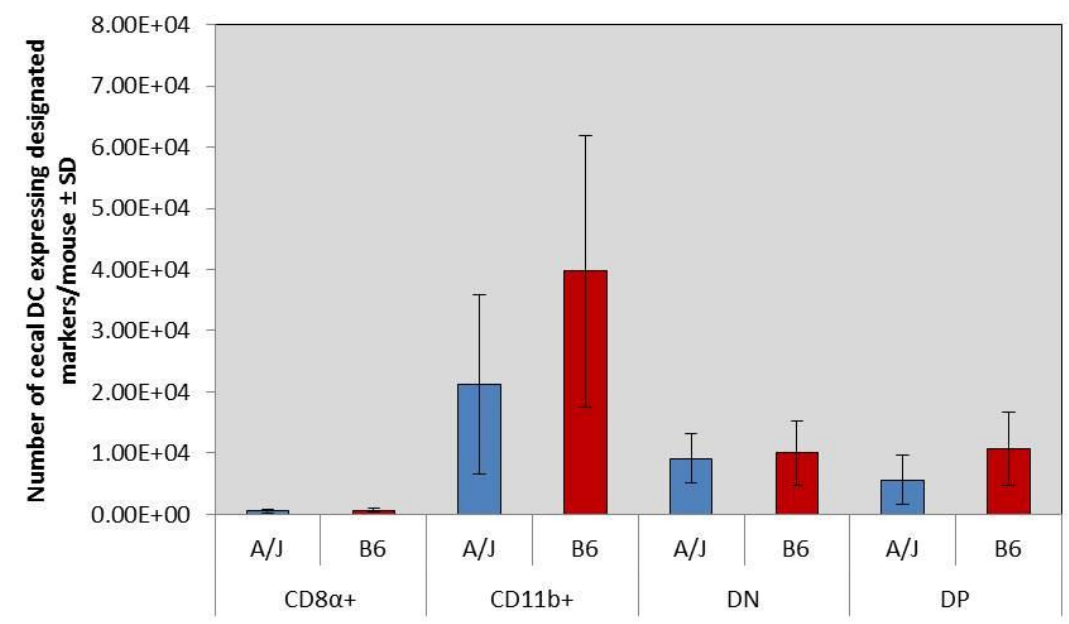

Figure 2.6 Number of cecal dendritic cells isolated per mouse from 90 day post-inoculation (90d PI) female $\mathrm{A} / \mathrm{J}$ and $\mathrm{C} 57 \mathrm{BL} / 6$ (B6) mice determined via flow cytometry to be CD8 $a^{+} C D 11 b^{-}$ $\left(C D 8 \alpha^{+}\right), \quad C D 8 \alpha^{-C D} 11 b^{+}\left(C D 11 b^{+}\right), C D 8 \alpha^{-C D} 11 b^{-}(D N)$, or $\mathrm{CD} \mathrm{a}^{+} \mathrm{CD} 11 \mathrm{~b}^{+}(\mathrm{DP}) \pm \mathrm{SD}(n=8-11)$. Asterisks represent significant difference $(p<0.05)$ as determined by Mann-Whitney rank sum test. 
IL-12/23p40 in naïve mice $(n=9-10)$

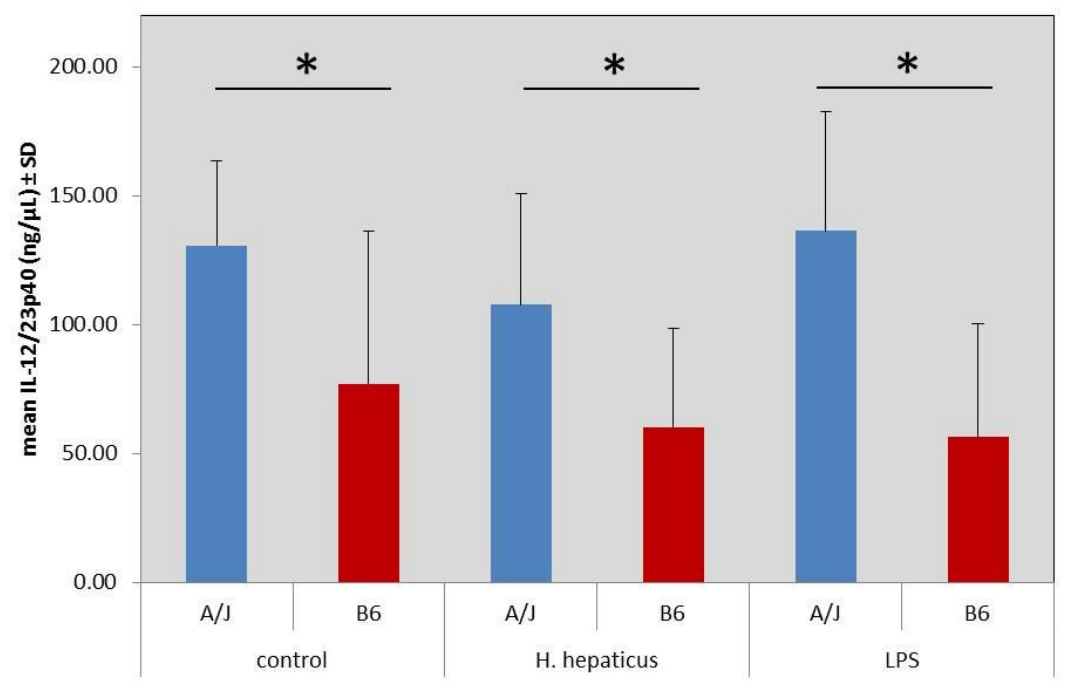

Figure 2.7 Mean IL-12/23p40 production from cells isolated from the cecum of naïve weanling $\mathrm{A} / \mathrm{JCr}(\mathrm{A} / \mathrm{J})$ and $\mathrm{C} 57 \mathrm{BL} / 6(\mathrm{~B} 6)$ mice and incubated 24 hours with the designated stimuli \pm standard deviation, as determined by enzyme-linked immunosorbent assay $(n=9-10)$. Asterisk represents significant difference $(p<0.05)$ as determined by two-way analysis of variance. 

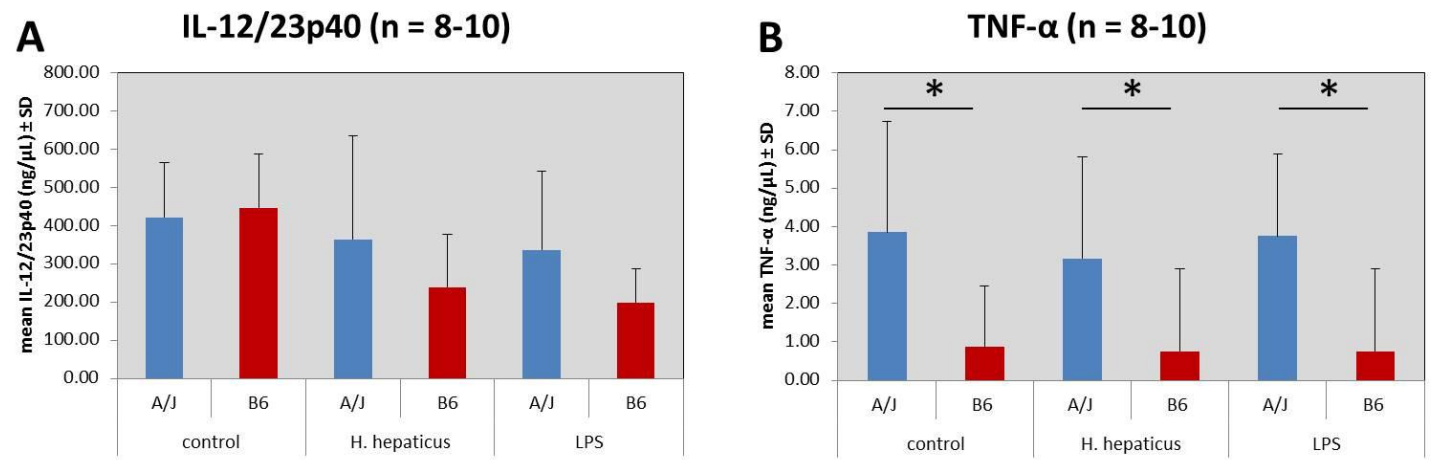

\section{CXCL10 in 4d PI mice $(n=8-10)$}

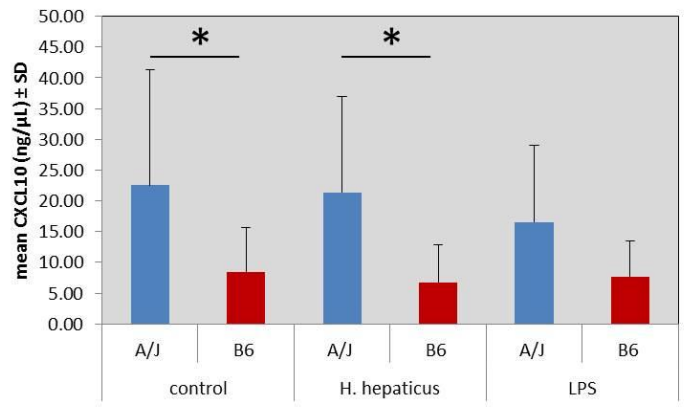

\section{CCL5 in 4d PI mice $(n=8-10)$}

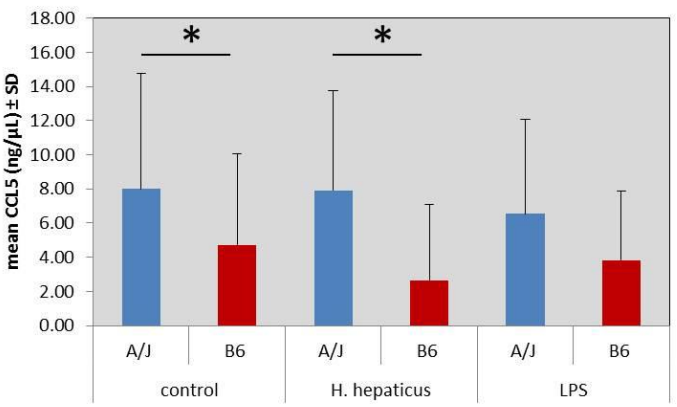

Figure 2.8 Mean IL-12/23p40 (A), TNF- $\alpha(B)$, CXCL10 (C), and CCL5 (D) production from cells isolated from the cecum of four day post-inoculation $\mathrm{A} / \mathrm{JCr}(\mathrm{A} / \mathrm{J})$ and $\mathrm{C} 57 \mathrm{BL} / 6$ (B6) mice and incubated 24 hours with the designated stimuli \pm standard deviation, as determined by enzymelinked immunosorbent assay (A) or microbead fluorescent immunoassay (B-D) $(n=8-10)$. Asterisks represent significant difference $(p<0.05)$ as determined by two-way analysis of variance. 
A IL-12/23p40 in 90d PI mice $(n=8)$

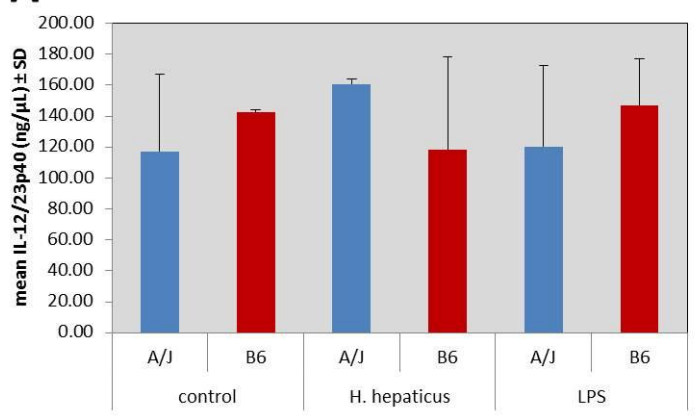

C ${ }_{12}$ CXCL10 in 90d PI mice $(\mathbf{n}=8)$

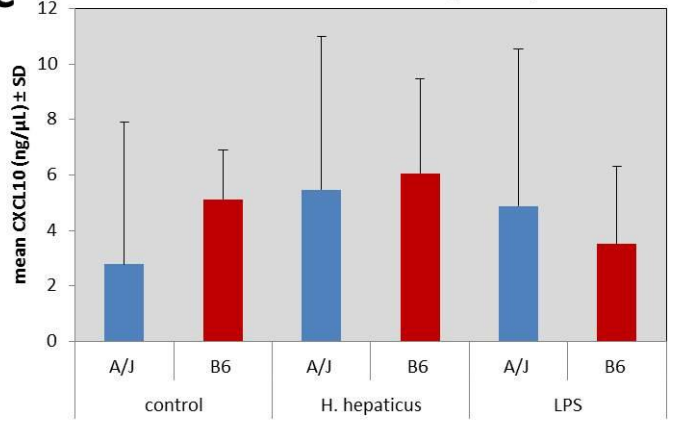

B TNF- $\alpha$ in $90 d$ PI mice $(n=8)$

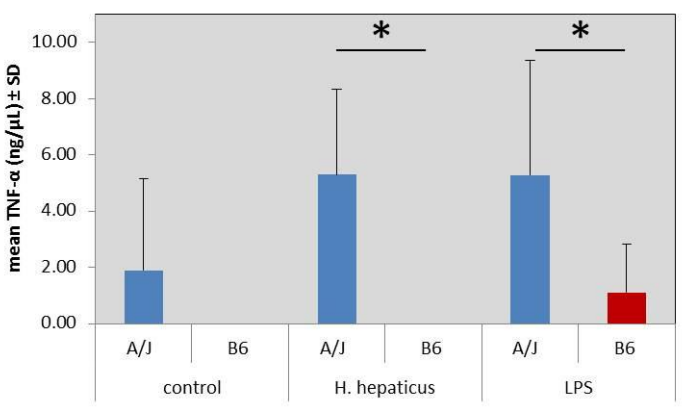

D CCL5 in 90d PI mice $(n=8)$

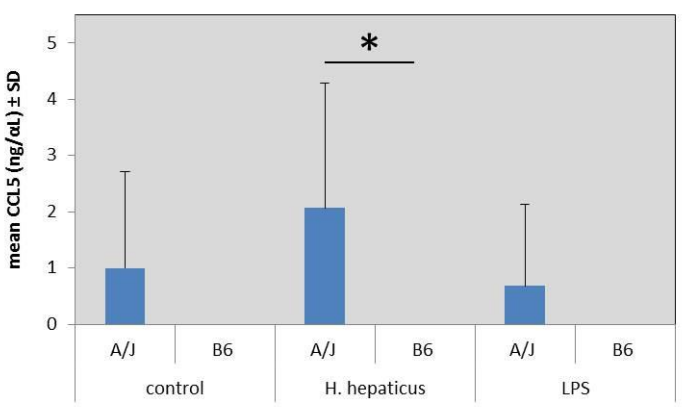

Figure 2.9 Mean IL-12/23p40 (A), TNF-a (B), CXCL10 (C), and CCL5 (D) production from cells isolated from the cecum of ninety day post-inoculation $\mathrm{A} / \mathrm{JCr}(\mathrm{A} / \mathrm{J})$ and $\mathrm{C} 57 \mathrm{BL} / 6$ (B6) mice and incubated 24 hours with the designated stimuli \pm standard deviation, as determined by enzymelinked immunosorbent assay (A) or microbead fluorescent immunoassay (B-D) $(n=8)$. Asterisks represent significant difference $(p<0.05)$ as determined by two-way analysis of variance. 

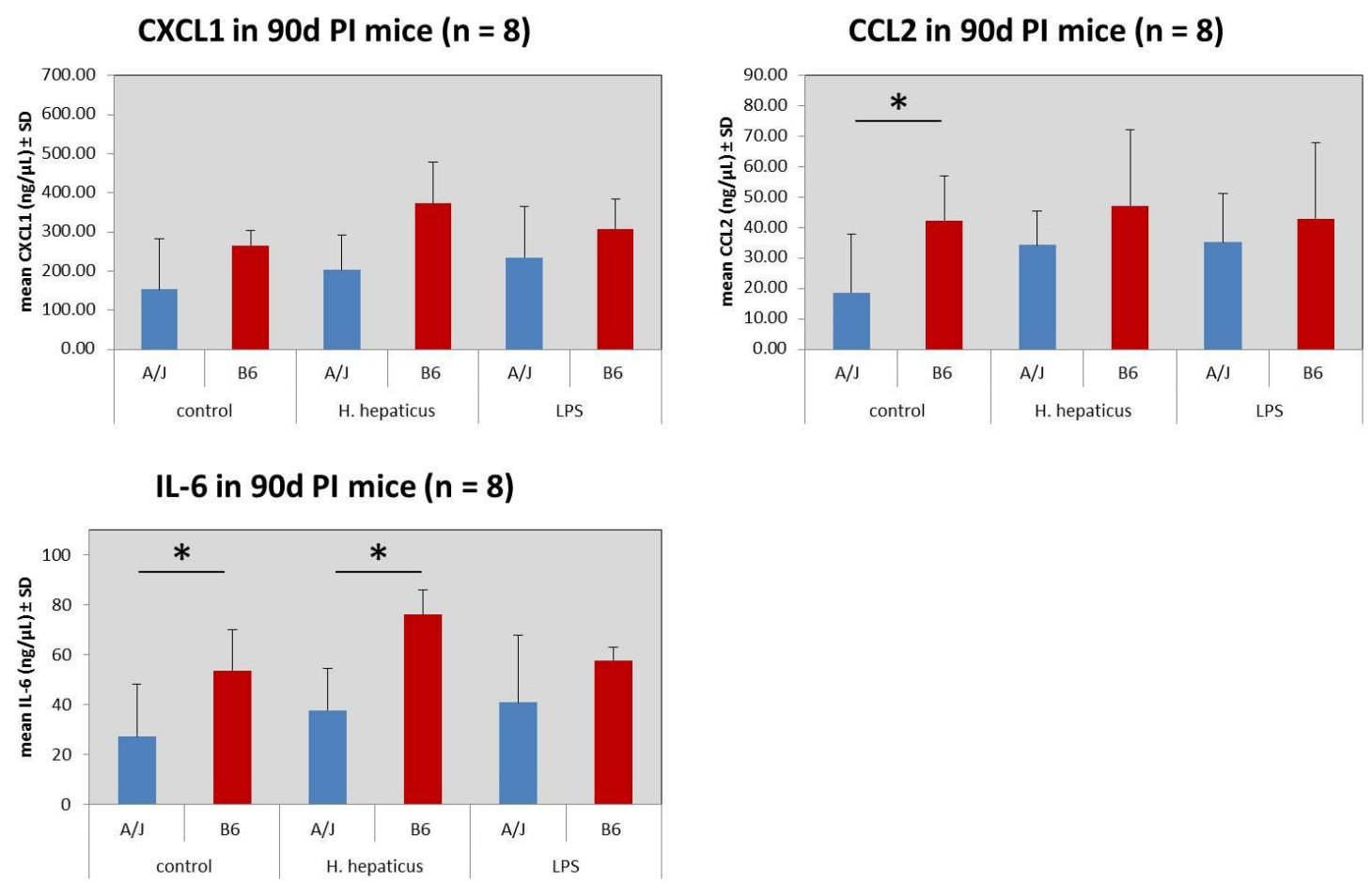

Figure 2.10 Mean CXCL1 (A), CCL2 (B), and IL-6 (C) production from cells isolated from the cecum of ninety day post-inoculation $\mathrm{A} / \mathrm{JCr}(\mathrm{A} / \mathrm{J})$ and $\mathrm{C} 57 \mathrm{BL} / 6$ (B6) mice and incubated 24 hours with the designated stimuli \pm standard deviation, as determined by microbead fluorescent immunoassay $(n=8)$. Asterisks represent significant difference $(p<0.05)$ as determined by twoway analysis of variance. 


\section{CHAPTER 3}

\section{OVERVIEW}

Colorectal cancer (CRC) is the second leading cause of cancer mortality in the U.S., and the third most common type of cancer in men and women ${ }^{227}$. Along with certain hereditary conditions, Inflammatory Bowel Disease (IBD) ranks as one of the top three high-risk conditions for $\mathrm{CRC}^{141}$. For IBD-associated CRC, there is no well-defined genetic etiology and CRC is thought to develop from a hyperplastic or dysplastic precursor lesion as a sequela to chronic inflammation. Classically, the stage of disease at diagnosis, as established by Dukes in $1932^{228}$, provides a prognostic indicator of survival $^{229}$ as resection prior to lymph node metastasis is often curative. Alternatively, diagnosis of CRC at Dukes' stage C or higher, indicating lymph node metastasis, is associated with poor five year survival rates, demonstrating the necessity of early detection. Currently, the most commonly utilized screening techniques are fecal occult blood testing (FOBT), sigmoidoscopy, colonoscopy, and CT colonography (often called virtual colonoscopy), all of which possess both advantages and disadvantages. FOBT is affordable and non-invasive but fails to detect most early pre-cancerous polyps and some cancerous lesions in humans ${ }^{230,231}$. The endoscopic techniques can be used to biopsy or completely remove potential lesions at the time of the procedure however they require cleansing of the colon, sedation in the case of colonoscopy, and there is an inherent risk, albeit minimal, of damage to the mucosa. Additionally, sigmoidoscopy is unable to detect lesions in the proximal colon ${ }^{232}$. CT colonography also requires cleansing of the colon. Mouse models of CRC are posed with similar challenges, particularly in the 
setting of therapeutic studies. As in humans, FOBT testing is limited by the same lack of robust sensitivity and specificity, and while colonoscopy is possible in mice ${ }^{233,234}$, it is both time- and cost-intensive, and requires anesthesia and substantial expertise.

Helicobacter-infected Smad3-knockout mice represent an attractive animal model for the study of CRC. Mice deficient in Smad3, a transcription factor downstream of the antiinflammatory and pro-apoptotic cytokine TGF- $\beta$, develop only a few mild phenotypic abnormalities including megaesophagus, an increased incidence of teratomas, and, at a very low incidence, angular limb deformities ${ }^{235}$ when raised in specific pathogen-free (SPF) conditions. However, when infected with enterohepatic species of Helicobacter, e.g. H. bilis or H. hepaticus ${ }^{156}$, approximately two thirds of these mice develop mucinous adenocarcinoma (MUC) of the proximal colon by as early as 6 weeks post-infection (PI). $H$. bilis and $H$. hepaticus induce intestinal inflammation in susceptible strains of mice ${ }^{182,}$ 236 and the neoplasia seen in Helicobacter-infected $\mathrm{Smad} 3^{-/-}$mice is considered a postinflammatory phenomenon ${ }^{156}$. Additionally, loss of normal TGF- $\beta$ signaling is widely recognized as an indicator of malignancy in human $\mathrm{CRC}^{237,238}$. Thus, the targeted deletion of Smad3 in mice is a biologically relevant model of the human condition.

This model is ideal for therapeutic studies of CRC in that the majority of mice develop disease on a predicted time course. Moreover, cancer develops in its natural setting as opposed to the more commonly used models that employ flank injections of immunocompromised mice with human cancer cell lines. To enhance the usefulness of this model, it would be ideal to be able to identify CRC at early stages of disease or even prior to disease onset. This would allow for both the elimination of mice from expensive therapeutic studies as well as the assessment of therapeutics at various stages of disease. 
For the purposes of evaluating therapeutic compounds for the treatment of CRC, we endeavored to establish a method of screening $\mathrm{H}$. bilis-infected $\mathrm{Smad}^{-/-}$mice prior to the time at which disease typically occurs. Our goal was to refine this mouse model of CRC in an effort to reduce animal numbers and the associated costs, and to increase the power of the data generated in these trials. Toward these ends, we evaluated both magnetic resonance imaging (MRI) and several fecal RNA biomarkers as means of detecting disease and predicting disease severity in $H$. bilis- and sham-inoculated Smad $3^{-/-}$mice in two separate, sequential longitudinal studies using different cohorts of mice. MRI was selected, as opposed to CT, based on the greater soft tissue definition afforded by this modality. Non-contrast enhanced MRI was able to detect MUC lesions beginning at 8 weeks PI and 58\% of mice with histologically confirmed lesions were correctly identified at 16 weeks PI. However, serial imagings produced inconsistent results. Additionally, MRI requires considerable resources to perform, and image interpretation is inherently subjective and requires expertise. Alternatively, fecal expression of mRNA specific for IL-1 $\beta$, MIP-1 $\alpha$, and RANTES at 3 weeks PI correlated significantly with MUC lesion severity at 9 weeks PI in H. bilis-infected $\mathrm{Smad}^{3^{--}}$mice, allowing the identification of which mice have a high probability of developing MUC. 


\section{MATERIALS \& METHODS}

Bacteria and Cultivation. A Helicobacter bilis isolate was obtained from an endemically infected mouse colony using a previously described culture technique ${ }^{186}$. The isolate was identified as $H$. bilis based on ultrastructural morphology, biochemical characteristics, and sequence analysis of the $16 \mathrm{~s}$ rRNA gene ${ }^{187}$. For inoculation, $H$. bilis cultures were grown in $5 \mathrm{~mL}$ of Brucella broth (Becton Dickinson, Franklin Lakes, NJ) supplemented with 5\% fetal calf serum (Sigma-Aldrich Co., St. Louis, MO) and overlaid on blood agar plates and incubated for 24 to 48 hours at $37{ }^{\circ} \mathrm{C}$ in a microaerobic environment containing $90 \% \mathrm{~N}_{2}, 5 \% \mathrm{H}_{2}$, and $5 \% \mathrm{CO}_{2}$.

Animals. All studies were performed in accordance with the Guide for the Care and Use of Laboratory Animals and were approved by the University of Missouri Institutional Animal Care and Use Committee. 129-Smad ${ }^{\text {tm/Par }} / \mathrm{J}$ (referred to as Smad3 $3^{-1}$ ) mice were bred on-site for these studies. Mice were confirmed to be free of adventitious viruses, parasites, and pathogenic enteric and respiratory bacteria, including all known murine Helicobacter spp. Three to four week old $\mathrm{Smad}^{3^{--}}$mice were inoculated twice, 24 hours apart, with $10^{8} \mathrm{H}$. bilis organisms in $0.5 \mathrm{~mL}$ Brucella broth, or an equivalent volume of sterile broth, via gastric gavage. Separate cohorts of mice were used for each study including weight of mice ( $n=9$ to 14 ; figure 3.1), incidence of CRC ( $n=5$ to 9 mice per time point; figures 3.3), MRI ( $n=6$ of each sex infected with H. bilis, and 1 of each sex sham-infected; figure 3.4), 2 to 9 week PI fecal 18s rRNA gene copies $(n=7 \mathrm{H}$. bilisinfected and 9 sham-infected; figure 3.5), 9 week PI fecal mRNA levels $(n=7 \mathrm{H}$. bilisinfected and 9 sham-infected; figure 3.6), 1 to 7 week PI fecal mRNA levels ( $n=14 \mathrm{H}$. bilis-infected and 9 sham-infected; figure 3.7), and Spearman's rank order correlation and 
receiver operating characteristic curves $(n=14 \mathrm{H}$. bilis-infected mice; figures 3.8 and 3.9). Mice were group-housed according to infection status in autoclaved microisolator cages and were provided autoclaved food and water. All manipulations and sample collections were performed in a biosafety hood except for MRI. Mice were euthanized at 16 or 9 weeks PI for the MRI and fecal biomarker studies respectively, via inhaled overdose of $\mathrm{CO}_{2}$.

Magnetic Resonance Imaging. MRI was performed at 3, 5, 8, 10, 12, 14, and 16 weeks PI using a 7T/210mm Varian Unity Inova MRI system equipped with a quadrature driven birdcage coil (38mm I.D.) (Varian Inc., Palo Alto, CA). Mice were anesthetized with 1$2 \%$ isoflurane in oxygen via a nose cone. A respiratory sensor was placed on the abdomen for monitoring of vital signs; body temperature was supported with warm air circulating in the magnet bore (SA Instruments Inc., Stony Brook, NY). Coronal and axial planes were collected using a spin echo $\mathrm{T}_{1}$-weighted imaging sequence with a fatsaturation pulse applied to suppress the strong signals from fatty tissues. Typically, images were collected with 21 slices, $0.8 \mathrm{~mm}$ slice thickness, pixel resolution of $59 \mathrm{~mm} \mathrm{x}$ $127 \mathrm{~mm}$ (coronal planes) and $59 \mathrm{~mm}$ x $59 \mathrm{~mm}$ (axial planes), and 4 scans to average the motion artifacts. Images were processed using VnmrJ (Varian Inc., CA).

Sample Collection and Experimental Design. For collection of fecal samples, mice were individually placed in autoclaved cages containing no bedding within a biosafety hood. Fecal pellets were collected at 1, 3, 5, 7, and 9 weeks PI with a sterile tuberculin syringe and placed in $200 \mu \mathrm{L}$ RNAlater ${ }^{\circledR}$ (Ambion, Austin, TX) for isolation of RNA. Pellets in RNAlater® were homogenized with a TissueLyser (Qiagen Inc., Valencia, CA), centrifuged briefly (Marathon 16km, Fisher Scientific, Pittsburgh, PA), and then 
vortexed to resuspend fecal material. Following euthanasia, the intestinal tract from ileum to rectum was collected and fixed in formalin.

Histopathology and Ranking of Lesions. Formalin-fixed tissues from H. bilis- and sham-infected mice were embedded in paraffin, cut in $5 \mu \mathrm{m}$-thick sections, and processed for staining with hematoxylin and eosin. CRC lesions in $H$. bilis-infected mice were ranked in a blinded fashion by two laboratory animal pathologists (AE and CF) according to lesion severity, based on the number of lesions, the longitudinal and vertical extent of neoplastic lesions, and the degree of associated inflammation. Rankings were compared and, in the case of discrepancies in ranking, pathologists conferred and agreed upon a rank order.

RNA Extraction from Feces. Total RNA was extracted using the RNeasy Mini Kit respectively, according to the manufacturer's protocols (Qiagen). RNA was quantified using a Nanodrop-1000 spectrophotometer (Nanodrop, Wilmington, DE) and quality was assessed by measuring the absorbance at 260 and $280 \mathrm{~nm}$.

Reverse Transcription. Five micrograms of total RNA were reverse transcribed using reverse transcriptase and oligo(dT) primers according to the manufacturer's protocol (SuperScript ${ }^{\circledR}$ First-Strand, Invitrogen, Carlsbad, CA). cDNA was diluted 1:1 with DPEC-treated water.

\section{Semiquantitative Real-time Reverse Transcriptase Polymerase Chain Reaction (RT-}

PCR). Semiquantitative real-time RT-PCR was used to measure mRNA levels in feces (LightCycler 1.5, Roche Diagnostic, Nutley, NJ). PCR reactions and melting curves were performed in a $20 \mu \mathrm{L}$ volume in glass capillaries containing $0.5 \mu \mathrm{M}$ of each primer, 
$3 \mathrm{mM} \mathrm{MgCl} 2$, QuantiTect SYBR Green PCR Master Mix (Qiagen), and cDNA. To quantify the number of copies of specific cDNA, a standard curve was created using known concentrations ( $10^{1}$ to $10^{6}$ copies) of the pCR4-TOPO (Invitrogen) plasmid containing the transcript of interest. PCR reactions were incubated at $95{ }^{\circ} \mathrm{C}$ for 15 minutes to activate the polymerase followed by 40 cycles consisting of a 15 -second denaturing at $94{ }^{\circ} \mathrm{C}, 20$-second annealing (see Table 1 for primer specific annealing temperature), and a 30 -second extension at $72{ }^{\circ} \mathrm{C}$. The ramp rate was $3{ }^{\circ} \mathrm{C} / \mathrm{s}$ for annealing and $20^{\circ} \mathrm{C} / \mathrm{s}$ for all other steps. Fluorescence was monitored at the end of each extension phase. Following amplification, melting curves were generated to confirm PCR product identity.

Primer Sequences and Plasmids. The sequences for HPRT ${ }^{239}, 18 \mathrm{~s}$ rRNA ${ }^{131}, \mathrm{IL}-1 \beta^{240}$, MCP- $2^{241}$, and MIP- $1 \alpha^{131}$, have been previously reported in the literature. The primer sequence for RANTES was designed from published mRNA sequences using DS Gene software (Accelrys, San Diego, CA). Primer sequences are listed in Table 1. Standards were generated using linearized plasmids containing cloned amplicons of selected targets using the Topo TA PCR-cloning kit (Invitrogen, Carlsbad, CA). Transcripts were quantified by comparing fluorescence of experimental samples to that of plasmid standards containing known concentrations of the cloned product.

\section{STATISTICAL ANALYSES}

Semiquantitative real-time RT-PCR. Semiquantitative real-time RT-PCR was used to measure mRNA levels in feces. The expression of IL-1 $\beta$, MIP-1 $\alpha$, MCP-2, and RANTES were normalized to expression of the house-keeping gene HPRT. The significance of 
differences in normalized expression levels between $\mathrm{H}$. bilis-infected $(n=7)$ and shaminfected $(n=9) \mathrm{Smad}^{-/-}$mice at 9 weeks PI was determined using the Mann-Whitney Rank Sum Test. The significance of differences between $H$. bilis-infected MUC+ $(n=$ 11), $H$. bilis-infected MUC- $(n=3)$, and sham-infected mice $(\mathrm{n}=9)$ at 1 to 7 weeks PI was determined using the Kruskal-Wallis one way ANOVA on Ranks and Dunn's method of multiple pairwise comparisons.

Correlation to Lesion Ranks. Coli were evaluated histologically and ranked according to lesion severity.Rank order of lesion severity in $\mathrm{H}$. bilis-infected $\mathrm{Smad}^{-/-}$mice was correlated to the rank order of normalized expression of each biomarker using Spearman Rank Order Correlation (SROC).

Receiver-operator Characteristic (ROC) Curves. Based on the presence or absence of CRC on histological interpretation, ROC curves were generated from H. bilis-infected (n = 14) $\mathrm{Smad}^{-{ }^{-}}$mice using the statistical software package SigmaPlot (SPSS, Chicago, IL). R software ${ }^{242}$ was used to compute the confidence intervals for the area under the curve (AUC) based on DeLong's method ${ }^{243}$ as well as to compare ROC curves to each other based on the Hanley and McNeil method ${ }^{244}$. For confidence intervals for the AUC, upper limits were truncated at unity. 


\section{RESULTS}

Progression of colon cancer in $\mathrm{Smad}^{-/-}$mice. $\mathrm{Smad}^{-/-}$mice on a $129 / \mathrm{Sv}$ background develop colonic neoplasia but this phenotype is dependent upon infection with either $H$. bilis or H. hepaticus, with tumors developing most often in the proximal colon ${ }^{155,156}$. Clinically, mice appeared completely normal and show no external evidence of disease such as hunched posture, scruffy haircoat, lethargy, or abnormal stool. Mice were also apparently eating and drinking normally as individuals infected with $H$. bilis gained weight at the same rate as sham-inoculated control mice (figure 3.1). In the present study, the tumors were typically single masses although less than $10 \%$ developed a second mass at other sites in the colon. Grossly, tumors appeared as either a thickened, pale area of the proximal colon, or as pearlescent, lobulated, exophytic masses reflecting the abundant mucin production seen in most masses (figure 3.2A). Histologically, these tumors were best classified as MUC, with an appearance similar to that seen in humans. Tumors were characterized by marked goblet and epithelial cell hyperplasia with extensive production of mucus, often seen sequestered in large dilated "mucin lakes", spilling into the lumen of the GI tract or penetrating the serosal surface and spilling into the peritoneal space (figure 3.2B-D). In the latter case, peritonitis was not uncommon. Many of the neoplastic epithelial cells retained a simple tall columnar morphology with centrally located, oblong nuclei containing a vesicular chromatin pattern. There were also abundant goblet cells, often approaching a 1:1 ratio with columnar epithelial cells. Surrounding the accumulations of mucinous material, the epithelium was variably attenuated, and sloughed epithelial and inflammatory cells were seen in the mucinous material, which was characterized by a lightly basophilic, homogenous to lacy 
appearance. There were also mild to moderate mixed inflammatory infiltrates in the areas around the tumor. Mitotic figures ranged from 0 to 4, with an average of 1 per HPF (400x). In the absence of Helicobacter infection, $\mathrm{Smad}^{-/-}$mice did not develop MUC or any detectable intestinal inflammation.

Incidence of MUC in $\mathrm{Smad3}^{-/-}$mice. To determine if the incidence of MUC would increase with increasing duration of infection, $\operatorname{Smad}^{-/-}$mice orally infected with $H$. bilis were sacrificed at multiple time points post-infection and the GI tract was collected for histological examination. Tissues were nominally classified as being neoplastic (possessing a characteristic MUC lesion), hyperplastic (showing evidence of focal or diffuse epithelial hyperplasia but not neoplasia), or lesion-free. While $100 \%$ of mice (5/5) examined at 9 weeks PI had developed characteristic MUC lesions, only $40 \%$ of mice $(2 / 5)$ at 11 weeks PI and $88 \%$ of mice (7/8) at 13 weeks PI showed histological evidence of neoplasia (figure 3.3). This was not a function of the early time point as similar results were obtained with mice at 20 weeks PI (data not shown). Thus, while the majority of mice developed identifiable MUC by as early as 6 weeks PI, not all mice progressed to MUC regardless of the duration of infection.

MRI detection of MUC in $\operatorname{Smad3}^{-/-}$mice. H. bilis-infected $\operatorname{Smad}^{-/-}$mice $(n=12,6$ male and 6 female) and naïve wild-type mice of the same background strain (1 male, 1 female) were imaged using MRI without contrast at 3, 5, 8, 10, 12, 14, and 16 weeks PI. Both coronal and axial images were collected at $0.8 \mathrm{~mm}$ intervals resulting in a total of approximately 14 and 18 images respectively needed to completely visualize the abdominal contents of one mouse. Immediately after the final imaging, mice were euthanized, and carefully dissected and photographed without disturbing the position and 
orientation of abdominal contents in situ. Gross findings were then compared to the 16 week PI images to assess the capacity of MRI to detect intestinal lesions and evaluate the level of background signal in control mice. Additionally, images from earlier imaging sessions were reviewed to determine if lesions at necropsy could be detected retrospectively. The earliest time point at which Helicobacter-infected mice were interpreted as possessing a neoplastic lesion was 8 weeks PI. While we were able to identify a reasonable agreement between the final (16 week PI) images and gross necropsy findings in 7 of $12 \mathrm{H}$. bilis-infected mice (sensitivity $=58.33 \%$ ) (figure 3.4), 5 of 12 mice with histologically identifiable MUC were interpreted as MUC-negative at 16 weeks PI.

\section{Total fecal DNA and 18s rRNA as a biomarker of MUC at 9 weeks PI in $H$. bilis-}

infected Smad3 ${ }^{-/-}$mice. To determine if the incidence of MUC could be detected via DNA or RNA-based fecal biomarkers, total DNA was isolated from H. bilis-infected and sham-inoculated mice. DNA was present in significantly greater quantities in the feces of H. bilis-infected mice, however the amount of fecal DNA isolated at any time point postinoculation failed to correlate with lesion severity at necropsy. As DNA isolated from feces may be of host or microbial origin, we also wanted to determine whether the amount of host DNA shed in the feces, reflecting the rate of intestinal epithelial sloughing, provided useful information regarding the presence or severity of MUC. The number of copies of the eukaryotic 18s rRNA gene was thus assessed via real-time PCR and normalized to the amount of overall DNA isolated. Again, the normalized number of 18s rRNA gene copies present in the feces differed significantly between $H$. bilis-infected 
and sham-inoculated mice (figure 3.5) however no correlation between lesion severity and gene copy number at any time point was detected.

Fecal cytokine gene expression as a biomarker of MUC at 9 weeks PI in $H$. bilisinfected Smad3 ${ }^{-/}$mice. At 9 weeks PI, H. bilis-infected Smad3 ${ }^{-/-}$mice expressed significantly higher levels of IL-1 $(p=0.001$, Mann-Whitney Rank Sum test), MIP- $1 \alpha$ $(p=0.004)$, and RANTES $(p=0.003)$ (figures 3.6A-C) than sham-inoculated mice. However, no difference was detected in the expression of MCP-2 despite a trend toward elevated expression in $H$. bilis-infected mice (figure 3.6D). Two sham-inoculated mice expressed levels of MCP-2 comparable to even the highest expressing $H$. bilis-infected mice, thus MCP-2 was eliminated from further experiments. In this cohort of mice, all 7 Helicobacter-infected mice developed histologically identifiable MUC. Spearman's Rank Order Correlation (SROC) was then performed in order to correlate the expression of IL-1 $\beta$, MIP-1 $\alpha$, and RANTES with lesion severity in the $H$. bilis-infected mice. Correlation coefficients for IL-1 $\beta$, MIP- $1 \alpha$, and RANTES were $0.929(p<0.001), 0.536$ $(p=0.18)$, and $0.750(p=0.04)$ respectively, indicating significant correlation between lesion scores and expression of both IL-1 $\beta$ and RANTES. Based on the significant overall difference in expression between infected and control mice in MIP-1 $\alpha$ expression, along with the fact that the two mice with the lowest MIP-1 $\alpha$ mRNA levels also demonstrated the lowest lesion scores, we opted to retain MIP-1 $\alpha$ in subsequent studies. Additionally, the kinetics of chemokine and cytokine expression vary, and we reasoned that at earlier time points, MIP-1 $\alpha$ might still prove to be a useful biomarker, despite poor overall correlation at 9 weeks PI. 


\section{Fecal cytokine gene expression during the progression of MUC in $\mathrm{H}$. bilis-infected}

$\mathbf{S m a d 3}^{-/-}$mice. To determine if fecal mRNA levels of IL-1 $\beta$, MIP-1 $\alpha$, and RANTES at time points earlier than 9 weeks PI could predict subsequent disease occurrence or severity, mice were inoculated as before with $H$. bilis $(n=14)$ or sterile broth $(n=9)$ and fecal samples were collected every two weeks starting at 1 week PI and continuing until 7 weeks PI. Mice were euthanized at 9 weeks PI and tissues were collected for histological examination. The normalized expression of each biomarker was determined at each time point, revealing similar kinetics for all three biomarkers (figure 3.7). Expression of all three biomarkers peaked at 1 week PI and then steadily declined thereafter in Helicobacter-infected mice. Nonetheless, even at 7 weeks PI, there was a significant difference (Mann-Whitney Rank Sum Test, $p<0.05$ ) between H. bilis-infected MUC ${ }^{+}$ and sham-infected mice for all three biomarkers.

To assess the value of each biomarker and determine the optimal screening paradigm, expression at each time point was correlated to lesion rank at 9 weeks PI using SROC. Additionally, receiver-operator characteristic (ROC) curves were generated to establish sensitivity, specificity, and appropriate cut-off values for each biomarker. SROC analysis of samples from 1 to 7 weeks PI revealed a significant correlation $(p<0.05)$ between lesion severity at 9 weeks PI and expression of IL-1 $\beta$ at 1, 3, and 5 weeks PI, MIP-1 $\alpha$ at 3 and 5 weeks PI, and RANTES at 3 weeks PI, indicating 3 weeks PI may be the optimal time for testing mice (figure 3.8). Surprisingly, the correlation between lesion rank and the expression of all three biomarkers was not statistically significant at 7 weeks PI. However, SROC indicates the overall agreement between lesions and the selected biomarkers across the entire range of lesion severity. As our goal was to identify "poor 
responders" and eliminate mice at the low end of the disease spectrum, ROC curves at each time point were compared to determine if very high specificity (>0.98) and acceptable sensitivity $(>0.80)$ could be achieved simultaneously. Considering the poor correlation for all markers at 7 weeks PI, empirical ROC curves were produced for only 1, 3, and 5 weeks PI (figure 3.9). Confidence intervals were established using DeLong's method $^{245}$ and the upper limit was truncated at one, as area under the curve (AUC) values, by definition, cannot exceed unity. All three biomarkers examined provided an estimated AUC of 0.97 at 3 weeks PI (figure 3.9D-F; 95\% CI $=0.89$ to 1). At 5 weeks PI however, fecal mRNA levels of IL-1 $\beta$ yielded an estimated AUC of 1, thus providing, in this sample, perfect sensitivity and specificity in predicting the presence or absence of MUC in mice at 9 weeks PI (figure 3.9G). However, ranking the relative performance of IL-1 $\beta$ at 5 weeks PI and IL-1 $\beta$, MIP-1 $\alpha$, or RANTES at 3 weeks PI is difficult because the small sample size gives limited power to discern differences in AUC; not surprisingly, none of the markers shown in figure 3.9 were statistically different from each other (smallest $p$-value $=0.1$ ), using the method of Hanley and McNeil ${ }^{246}$. As the goal of this study was to establish a screening method with the ability to predict which mice would not develop CRC as a means of conserving resources, it should be noted that by as early as 1 week PI, IL-1 $\beta$ provided an AUC of $0.97(95 \% \mathrm{CI}=0.89$ to 1$)$, the same as all three biomarkers at 3 weeks PI. Additionally, the overall correlation between lesion severity rank at 9 weeks PI and fecal mRNA levels of IL-1 $\beta$ at 1 week PI was 0.65 ( $p=0.01 ;$ Spearman rank order correlation). Thus we propose that in this model of MUC, RT-PCR analysis of fecal mRNA specific for IL-1 $\beta$ at 1 week PI, or of IL-1 $\beta$, MIP-1 $\alpha$, or RANTES at 3 weeks PI is a reliable, non-invasive determinant of which mice 
will not progress to MUC. More importantly, this provides proof-of-principle that fecal nucleic acid analysis has utility in mouse models of gastrointestinal neoplasia, as a means of reducing the number of animals used in study. This may also portend novel screening methods of interest to the human population. 


\section{DISCUSSION}

The World Health Organization defines MUC in humans as a tumor with $>50 \%$ showing a mucinous pattern on histological examination, and with a large amount of extracellular mucin produced by secreting acini ${ }^{247}$. Of colorectal cancer, MUC account for between 11 and $15 \%{ }^{248}$. Helicobacter-infected $\mathrm{Smad}^{-{ }^{--}}$mice recapitulate the human condition faithfully, with extensive mucin production seen multifocally in neoplastic foci, forming dilated pockets of mucinous material extending into the lumen and frequently through the tunica muscularis to the serosal surface of the GI tract. There are several reasons to believe that the mechanisms leading to MUC in humans and Helicobacter-infected $\mathrm{Smad}^{-{ }^{--}}$mice are similar. In humans, as in Helicobacter-infected Smad $3^{-/-}$mice, MUC occurs more frequently in the proximal colon than elsewhere in the GI tract ${ }^{249-251}$. While not all studies agree, possibly due to geographical differences ${ }^{252}$ or the presence of two subtypes of colorectal $\mathrm{MUC}^{249}$, MUC in humans appears to be more prevalent as a sequela to IBD than as spontaneous CRC not associated with prior IBD ${ }^{142,251,253-255}$. Supporting this concept, MUC occurs primarily in areas of chronic inflammation and the risk of MUC increases with duration of $\operatorname{IBD}^{256,257}$. Similarly, the findings detailed herein support the notion that the severity of MUC in Helicobacter-infected Smad3 ${ }^{-{ }_{-}}$mice is largely dependent on the robustness of the inflammatory response to a member of the gut flora, as measured by the expression of certain fecal cytokines and chemokines. Additionally, MUC in humans is frequently associated with fistula formation ${ }^{251,254,258-261}$, a phenomenon attributed to adenomatous transformation of the epithelium lining the fistula tract ${ }^{262}$. Many H. bilis-infected $\mathrm{Smad}^{-/-}$mice showed histologic evidence of perforation of the bowel wall and areas in which dysplastic epithelial cells are seen 
invading and penetrating the serosal surface (Figures 1B-D), and it is tempting to speculate that a similar mechanism is at work in the formation of MUC lesions in Smad3${ }^{\prime-}$ mice.

Regardless of the pathogenesis, not all $\mathrm{Smad}^{-/-}$mice will develop MUC despite persistent colonization with $H$. bilis. As a consequence, many of these MUC-resistant mice will be utilized in expensive therapeutic trials lasting up to 8 months in duration. Along with the time and money spent maintaining mice that will not progress to MUC, one must also consider that these mice are potentially confounding the research by making therapeutic compounds appear falsely efficacious. The ability to non-invasively identify which mice will not progress to cancer would both conserve resources and increase the power of data generated by using only mice with a high probability of developing MUC. We first evaluated MRI as a means of detecting early inflammatory or precancerous lesions in Helicobacter-infected Smad3 ${ }^{-/-}$mice. Mice were imaged every two to three weeks until 16 weeks PI, a time by which previous studies (Figure 2) had demonstrated the majority of mice would develop MUC. While MRI provided some diagnostic information, i.e. the presence or absence of a lesion, it afforded little prognostic information regarding the severity or extent of disease at necropsy when images were analyzed retrospectively. This is partially due to the variability between images from week to week. Frequently, MRI would indicate a possible lesion at one time point, followed by images at subsequent time points interpreted as negative. In only one of twelve mice did images consistently contain suspect lesions following the initial appearance. Additionally, in those mice in which a correlation between 16 week PI MRI images and gross lesions was detected, it was difficult to reliably track the course of 
intestinal neoplasms retrospectively. The background noise, seen in both experimental and control mice, was considerable and was most problematic in highly glandular tissue such as the reproductive tract. Additionally, the severity of the lesions could not be predicted based on the size and intensity of suspect lesions on MRI. Mice with hyperintense signal on the final imaging, indicating a large or severe lesion, often revealed mild or moderate MUC lesions at necropsy. Conversely, mice with borderline 'positive' final images often revealed extensive marked MUC or even multiple lesions. MRI has been applied to the human population as a screening method for MUC; however, the method requires insufflation of the colon with air to enhance imaging ${ }^{227}$. The lack of insufflation in our study may partially explain the lack of adequate definition with MRI. Also, the imaging in this study was performed without the use of contrast. MRI studies of the gastrointestinal tract using a fecal-tagging based MRI contrast agent may enhance visualization of MUC lesions ${ }^{263}$. The primary goal of these studies was to evaluate two distinct methods of detecting CRC in a mouse model, non-invasively and as early in the disease process as possible. As our motivation was to conserve resources, we opted to omit insufflation and contrast in an effort to keep the procedure as simple and cost-effective as possible. In our study, a mass showing hyperintense and heterogeneous signal contents would indicate a MUC lesion (Figure 3B and 3C). However, abdominal motion artifacts and significant signals from feces often cause ambiguities or missed detections. A respiratory-gated $\mathrm{T}_{2}$-weighted MRI protocol may be applied to increase the detection accuracy and specificity for MUC, due to the brighter signal nature of mucin contents in MUC lesions on $\mathrm{T}_{2}$-weighted images; however with the expense of prolonged imaging time. Lastly, considering the expense of the initial purchase, maintenance, and 
operation, MRI is a costly technique for screening large numbers of animals. Imaging in both coronal and axial planes resulted in 21 and 42 images respectively per mouse at each time point which, along with the user-dependent nature of image interpretation, made this a time-consuming and subjective technique. As our impetus for screening animals is to eliminate mice that are not going to develop MUC as a means of saving costs, MRI is problematic. Thus, the inability of MRI to detect disease in a reproducible manner, the associated costs, and the labor-intensive and subjective nature of this method, make it less than ideal for our purposes.

Next, we elected to determine if CRC in our model could be predicted through the analysis of fecal cytokine and chemokine message levels. This concept, while not new in humans $^{264,265}$, has not been applied to murine models to the authors' knowledge. As humans and mice both constitutively slough colonic epithelial cells in feces, the RNA isolated from these cells should reflect the state of health or inflammation present in the gut. Inflammation is now recognized as a risk factor and negative prognostic indicator for certain types of neoplasia in humans ${ }^{266}$. Adaptive antitumoral immune responses, particularly those mediated by $\mathrm{CD} 8^{+} \mathrm{T}$ cells, are considered protective and beneficial in destroying tumor cells, while inflammation due to innate immune responses is often associated with a poor prognosis ${ }^{267}$. This concept can be extrapolated to the chemokines responsible for attracting $\mathrm{T}$ cells or macrophages; accumulation of lymphocytes due to increased expression of CXCL16 correlates with a favorable outcome in human CRC $^{268}$, while accumulation of tumor-associated macrophages due to increased levels of CCL2 correlates with poor outcome ${ }^{269}$. Thus, as a means of non-invasively assessing the degree of colonic inflammation in $\mathrm{Smad}^{-/-}$mice, we measured the fecal levels of several factors 
involved in macrophage recruitment, and which have been shown to be elevated in human $\mathrm{CRC}^{267,270,271}$, including the chemokines MIP-1 $\alpha$, RANTES, and MCP-2, and IL$1 \beta$, a highly pleiotropic pro-inflammatory cytokine with effects on virtually all cell types $^{272}$. Our initial fecal biomarker study was performed at 9 weeks PI, when the majority of mice were expected to have developed MUC. It was established that a significant difference in the fecal expression of IL-1 $\beta$, MIP-1 $\alpha$, and RANTES existed between Helicobacter- and sham-infected mice (Figure 4), however 100\% (7/7) of the infected mice in this group developed MUC, making a comparison of MUC+ and MUCmice within the infected group impossible. Pursuing earlier time points PI (Figure 5) with a second group of mice provided evidence that there is also a significant difference in expression of these biomarkers between $H$. bilis-infected MUC+ and MUC- mice, supporting their use as predictors of MUC in this model. It is notable that the expression of IL-1 $\beta$, MIP-1 $\alpha$, and RANTES showed an acute increase following infection with Helicobacter, which waned steadily thereafter. While these biomarkers are primarily associated with innate immune responses, infection with $H$. bilis eventually induces an adaptive immune response ${ }^{80}$, allowing the innate response to wane accordingly. Like the selected chemokines, IL-1 $\beta$ is produced by the intestinal epithelium. As intestinal epithelial cells also express the activating receptor IL-1RI ${ }^{272}$, IL-1 $\beta$ functions in an autocrine and paracrine manner to amplify chemokine expression. Similarly, RANTES has been shown to stimulate production of MIP-1 $\alpha$ by human monocytes ${ }^{273}$, suggesting activation of tumor-associated macrophages may amplify recruitment of additional monocytes and other leukocytes, thought to be the source of harmful reactive oxidative intermediaries. Interestingly, several studies indicate that IL-1 $\beta$ may have a more direct 
role in colorectal tumorigenesis. In 2003, Liu et al. demonstrated that IL- $1 \beta$ upregulates the expression of cyclooxygenase $(\mathrm{COX})-2^{274}$, which is over-expressed in $80-90 \%$ of human $\mathrm{CRC}^{275}$, and was also found to be elevated in Helicobacter-infected Smad3 ${ }^{-/-}$mice relative to naïve mice ${ }^{156}$. Similarly, Maihöfner et al. showed that in both human CRCassociated neoplastic epithelium and tumor-associated macrophages, COX-2 expression was markedly increased and that increase correlated with increases in IL-1 $\beta^{276}$. COX-2 functions to facilitate tumor development through the induction of anti-apoptotic and angiogenic factors ${ }^{277,278}$, and it is worth noting that fecal mRNA levels of COX-2 have been evaluated as screening techniques for human $\mathrm{CRC}^{279,280}$. Additionally, IL-1 $\beta$ has a potent proliferative effect on human carcinoma cell lines ${ }^{281}$, most likely via the induction of other growth factors. With regard to the mucinous phenotype seen in Helicobacterinfected Smad3 ${ }^{-/-}$mice, IL-1 $\beta$ has been shown to upregulate the expression and release of mucins in both colonic epithelial cell lines ${ }^{282,283}$ and perfused rat colon ${ }^{284}$. Regardless of the mechanism, the present data suggest that MUC in $\mathrm{Smad}^{-/-}$mice is highly correlated with the prior expression of IL-1 $\beta$, MIP-1 $\alpha$, and RANTES.

Until recently, the amplification of nucleic acids from feces has been hindered by poor recovery due to the presence of nucleases. DNA is much more stable than RNA and can typically be amplified from feces that have been snap-frozen in liquid nitrogen. However, due to the inherent instability of RNA and the abundance of prokaryotic nucleases in the feces, a preservative containing RNAse inhibitors is needed to isolate mRNA from feces. When compared to several other methods of RNA preservation including liquid nitrogen, silica gel, Whatman FTA cards, and Paxgene, Yu et al. found RNAlater ${ }^{\circledR}$ to provide the optimal quantity and quality of RNA, as well as the lowest 
level of genomic DNA contamination. For our studies, two fecal pellets per mouse were collected in $200 \mu \mathrm{L}$ of RNAlater ${ }^{\circledR}$ at each time point, yielding 600 to $800 \mathrm{ng} / \mu \mathrm{L}$ RNA per sample. Assuming an average mass of $35 \mathrm{mg}$ per fecal pellet, this results in an average yield of approximately $70 \mu \mathrm{g}$ RNA per gram of feces. An early description of a similar technique applied to human stool yielded from 5-30 $\mu \mathrm{g}$ RNA per gram of stool from CRC patients and approximately $5 \mu \mathrm{g}$ RNA per gram from healthy controls ${ }^{264}$. Several points should be made regarding this difference. An obvious caveat is the different methods of RNA extraction from feces. While the present data represent RNA extracted using a commercially available kit from feces preserved in RNAlater®, the samples from human CRC patients and controls were snap frozen in liquid nitrogen, and RNA was extracted with acid phenol and chloroform. A second consideration regarding differences in RNA recovery from mouse and human stool is the fate of senescent epithelial colonocytes. In humans, effete colonocytes are primarily removed via mucosal phagocytosis, allowing subcellular components to be recycled ${ }^{285}$. Alternatively, colonocytes in rodents are lost primarily via simple exfoliation into the lumen ${ }^{285}$. Thus it would be expected that rodent feces would contain more RNA on a per weight basis and may be ideally suited to molecular techniques such as those described here.

There is a strong likelihood that these techniques are applicable to other mouse models of colorectal cancer. One would expect that post-inflammatory models of intestinal neoplasia would be particularly amenable to fecal cytokine or chemokine analysis. Several other strains of mice used as models of colitis are also prone to CRC, including

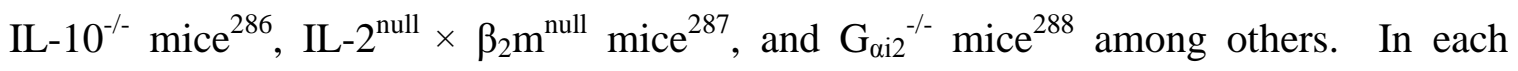
model, while the development of colitis precedes CRC, suggesting that CRC is driven by 
the inflammatory response, only 31 to $60 \%$ of mice in these models will progress to carcinoma $^{142}$. Thus, while the appropriate biomarkers and their kinetics would need to be established for each model (and possibly laboratory), the concept of predicting the presence or severity of disease through the use of fecal biomarkers of inflammation likely applies to more than just the Smad3 $3^{-/-}$mouse model. Similarly, several fecal biomarkers, including RNA specific for cyclooxygenase-2 and matrix metalloproteinase-7, have garnered interest as screening tools for CRC in humans ${ }^{279,}{ }^{280}$. As microarray technologies become more commonplace, the use of fecal RNA analysis may allow for robust, non-invasive CRC screening in humans. Smad $3^{-/-}$mice offer a useful tool for these types of studies and the refinement of the model described herein will enhance the development of both screening techniques and therapeutic modalities for CRC. 


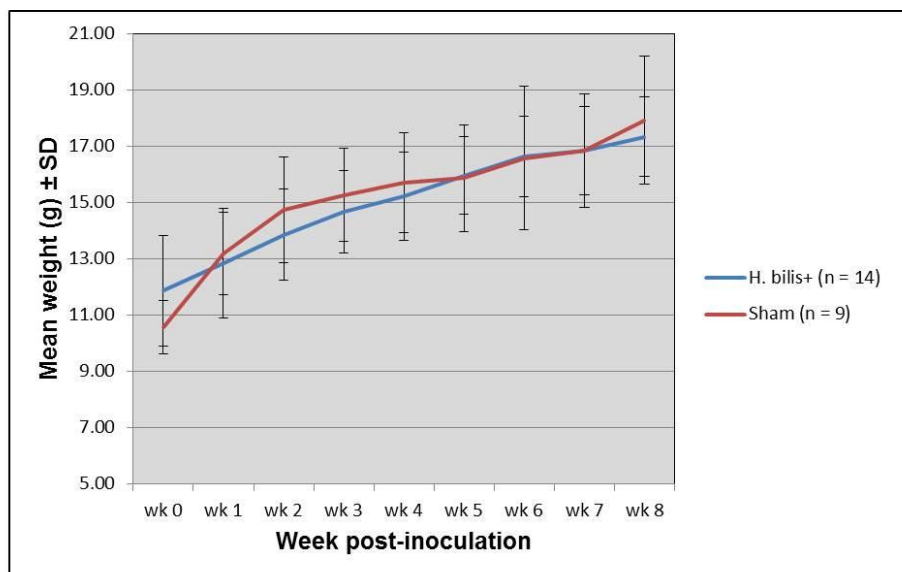

Figure 3.1 Mean weight in grams of $H$. bilis-infected (blue line; $n=14)$ ) and sham-inoculated (red line; $n=$ 9)) Smad3 ${ }^{-/}$mice from zero to eight weeks postinoculation \pm standard deviation. 


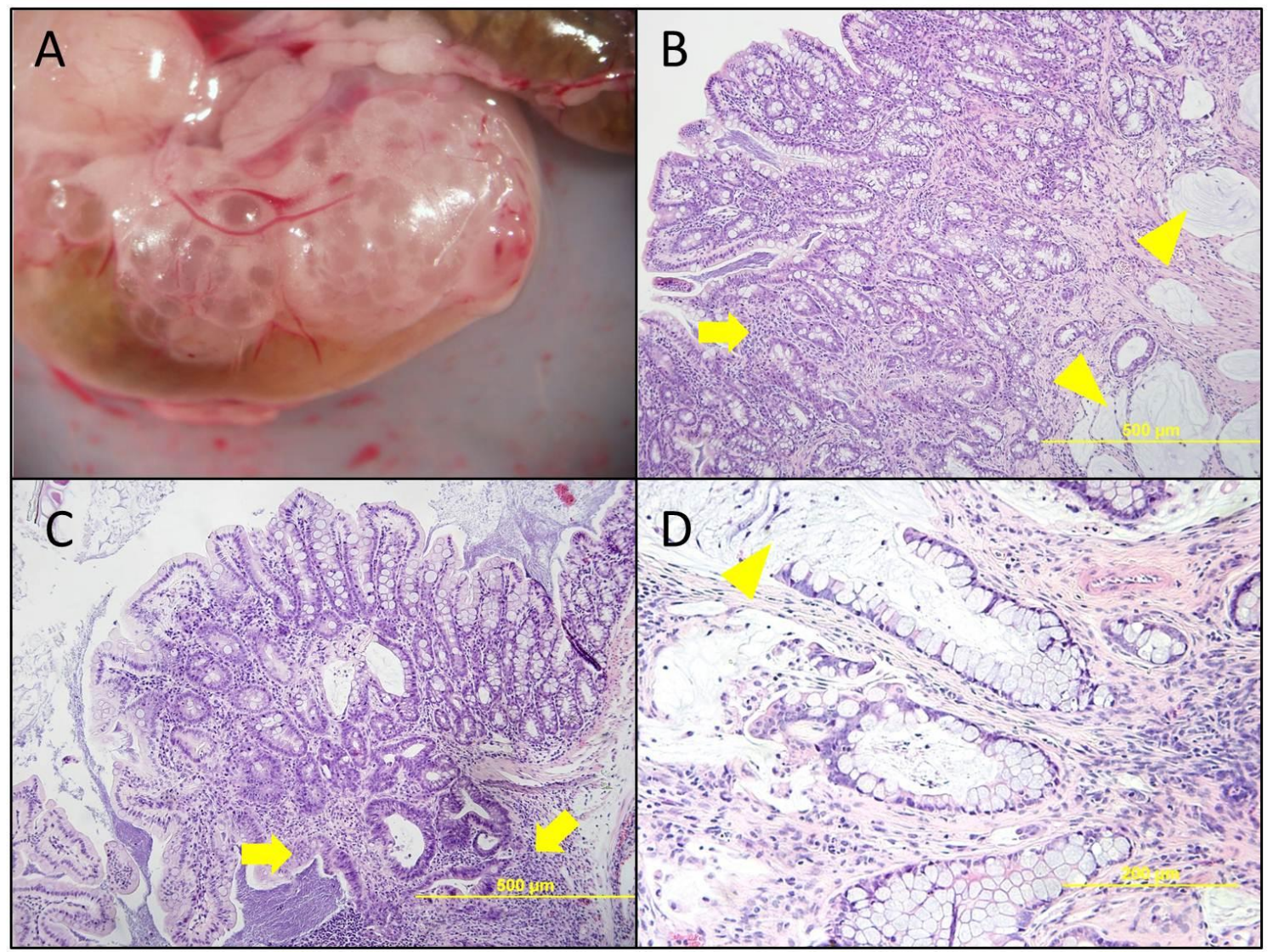

Figure 3.2 Representative images demonstrating the typical gross (A) and histological (B-D) appearance of mucinous adenocarcinoma of the proximal colon in $\mathrm{H}$. bilis-infected Smad3-/- mice at 9 weeks post-infection; Hematoxylin and eosin stain at 100× (B,C) and 200× (D) magnification. Arrows represent areas of inflammation; arrowheads mark accumulations of mucinous material, i.e. "mucin lakes". 


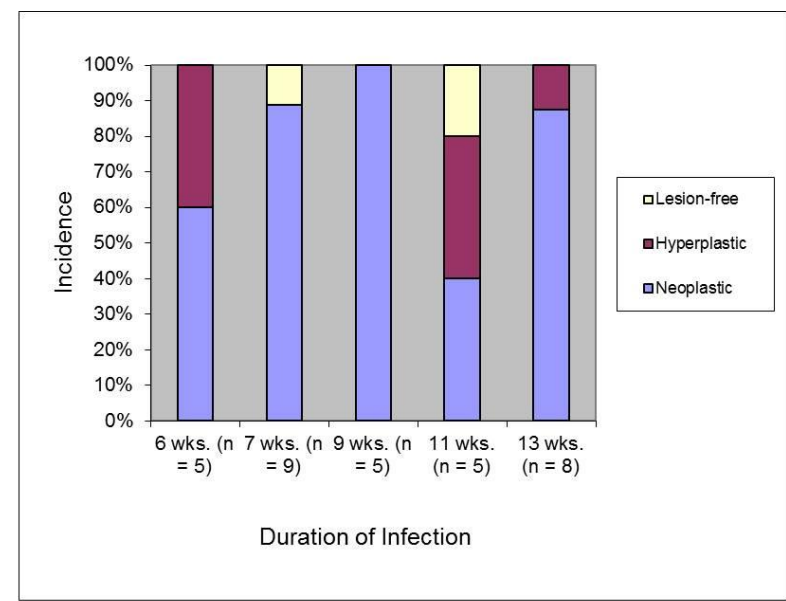

Figure 3.3 Incidence of neoplasia and hyperplasia in Smad3 ${ }^{--}$mice ( $n=5$ to 9 ) at increasing durations of infection with $H$. bilis 

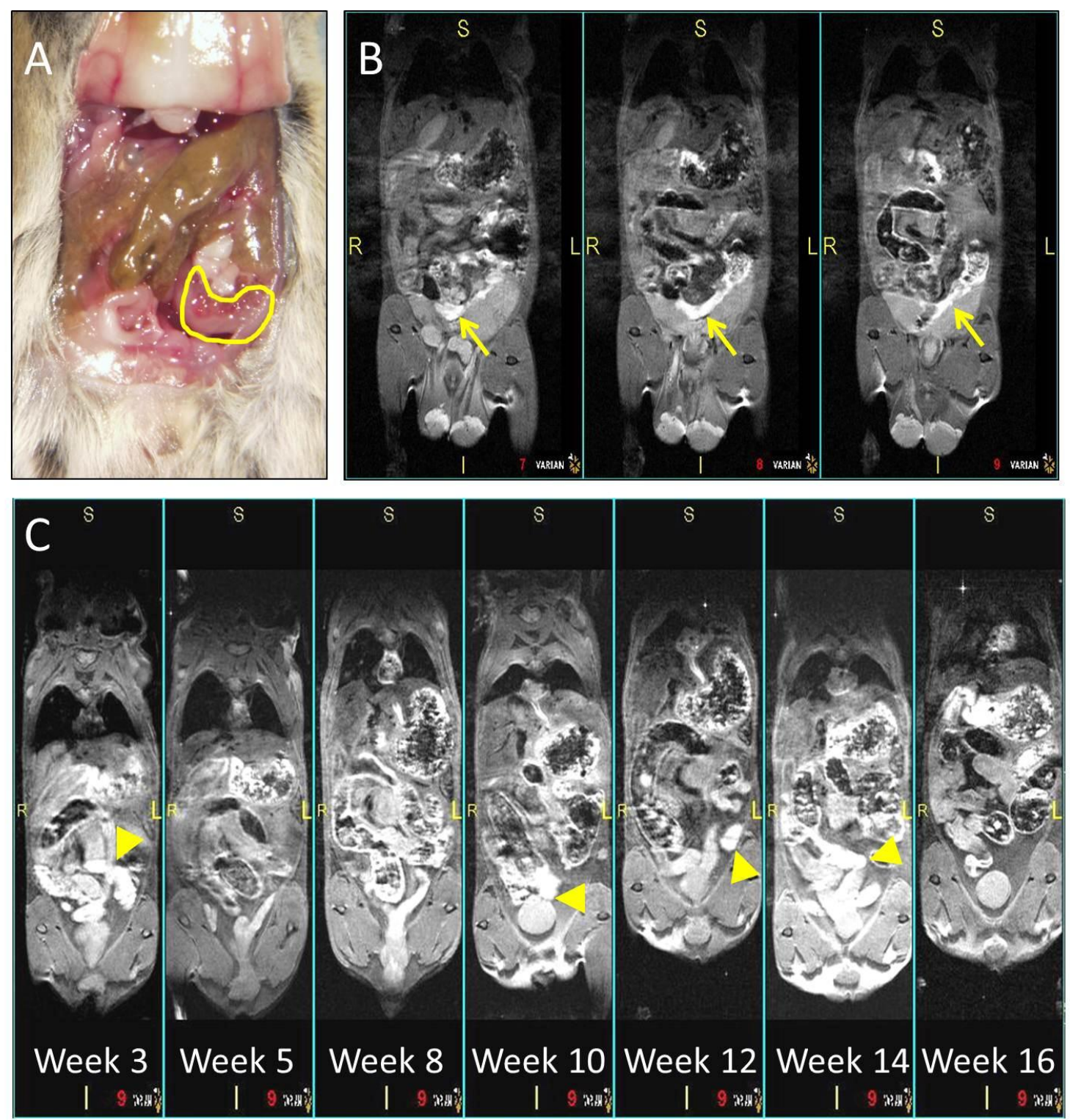

Figure 3.4 Appearance of mucinous adenocarcinoma in $H$. bilis-infected Smad3-mouse in situ; outlined (A) and consecutive coronal images of the same mouse at 16 weeks post-infection, demonstrating the corresponding lesion; arrows (B), serial coronal images of a female mouse from week 3 through week 16 post-inoculation, demonstrating background signal mimicking lesions; arrowheads (C). 


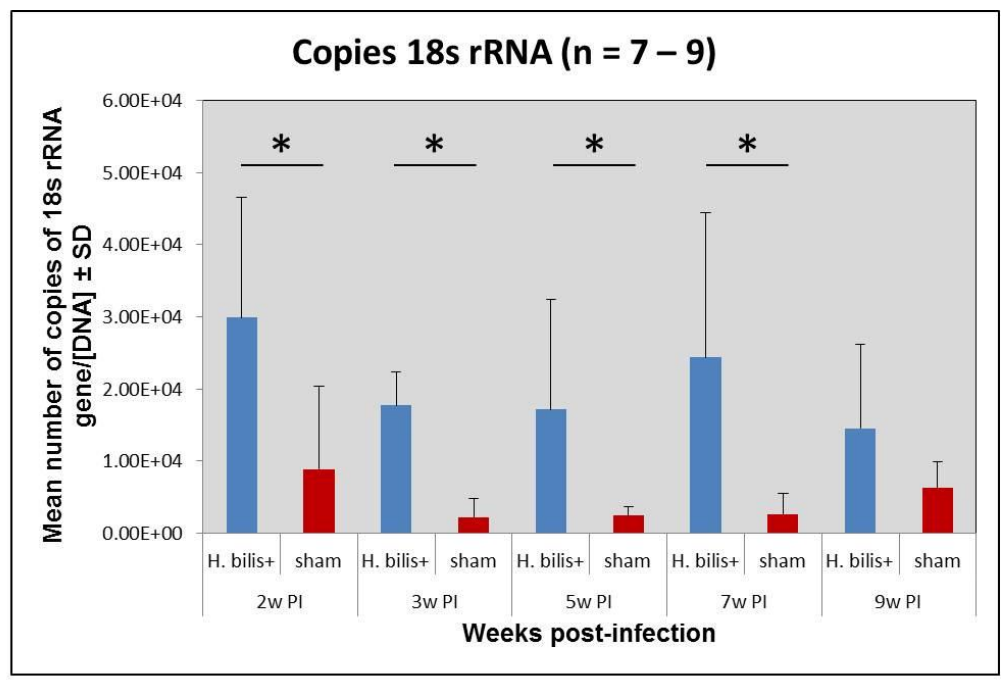

Figure 3.5 Mean number of 18s rRNA gene copies in the feces of $H$. bilis- and sham-inoculated Smad3 ${ }^{-/-}$mice ( $n=7-9)$ at $2,3,5,7$, and 9 weeks post-inoculation as determined by polymerase chain reaction, normalized to the overall concentration of fecal DNA \pm standard deviation; statistically significant $(p<0.05)$ differences between groups are denoted with an asterisk; MannWhitney rank sum test. 

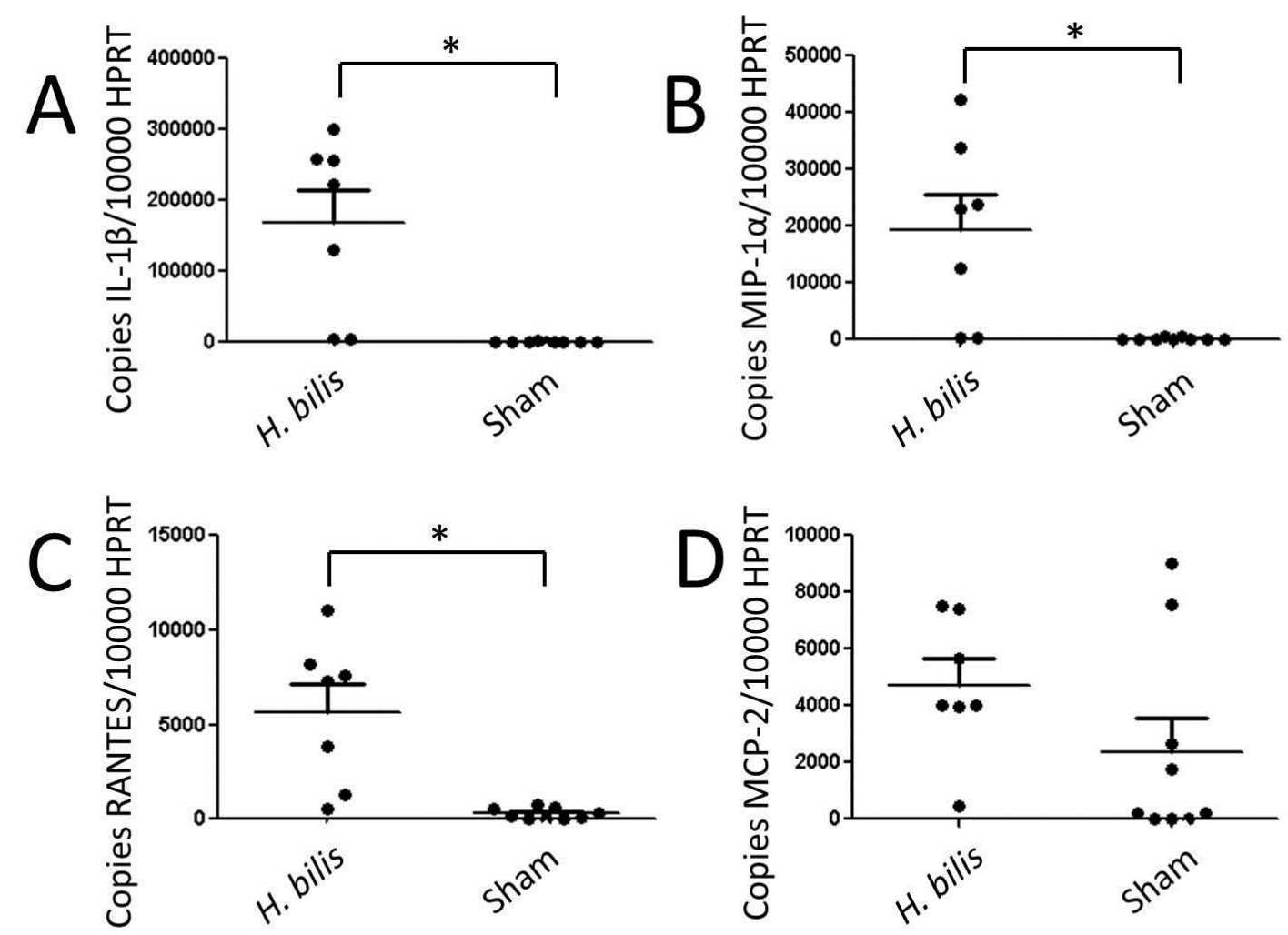

Figure 3.6 Measurement of IL-1 $\beta$ (A), MIP-1 $\alpha$ (B), RANTES (C), and MCP-2 (D) in the feces of $H$. bilis- and sham-inoculated Smad3-- mice $(n=7-9)$ at nine weeks post-inoculation as determined by semi-quantitative real-time reverse transcription polymerase chain reaction. Data are reported as the number of gene transcripts of interest relative to HPRT. Bars represent the mean and standard error of the mean. Statistically significant $(p<0.05)$ differences between groups are denoted with an asterisk; Mann-Whitney rank sum test. 

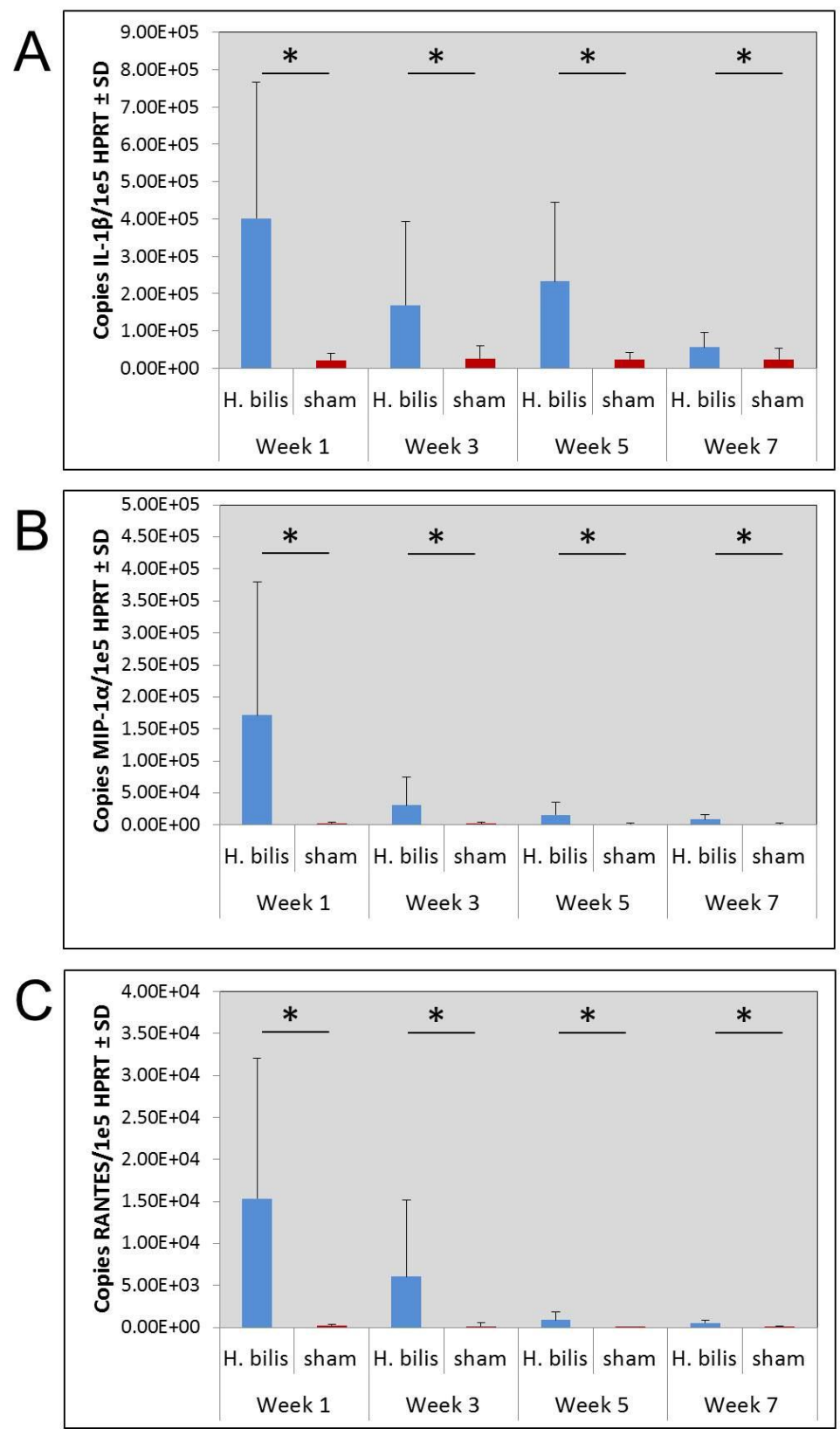

Figure 3.7 Measurement of IL-1 $\beta(A)$, MIP-1 $\alpha(B)$, and RANTES (C) in the feces of $H$. bilisand sham-inoculated Smad3 ${ }^{--}$mice $(n=9-14)$ at nine weeks post-inoculation as determined by semi-quantitative real-time reverse transcription polymerase chain reaction. Data are reported as the number of gene transcripts of interest relative to HPRT \pm standard deviation. Statistically significant $(p<0.05)$ differences between groups are denoted with an asterisk; Mann-Whitney rank sum test. 


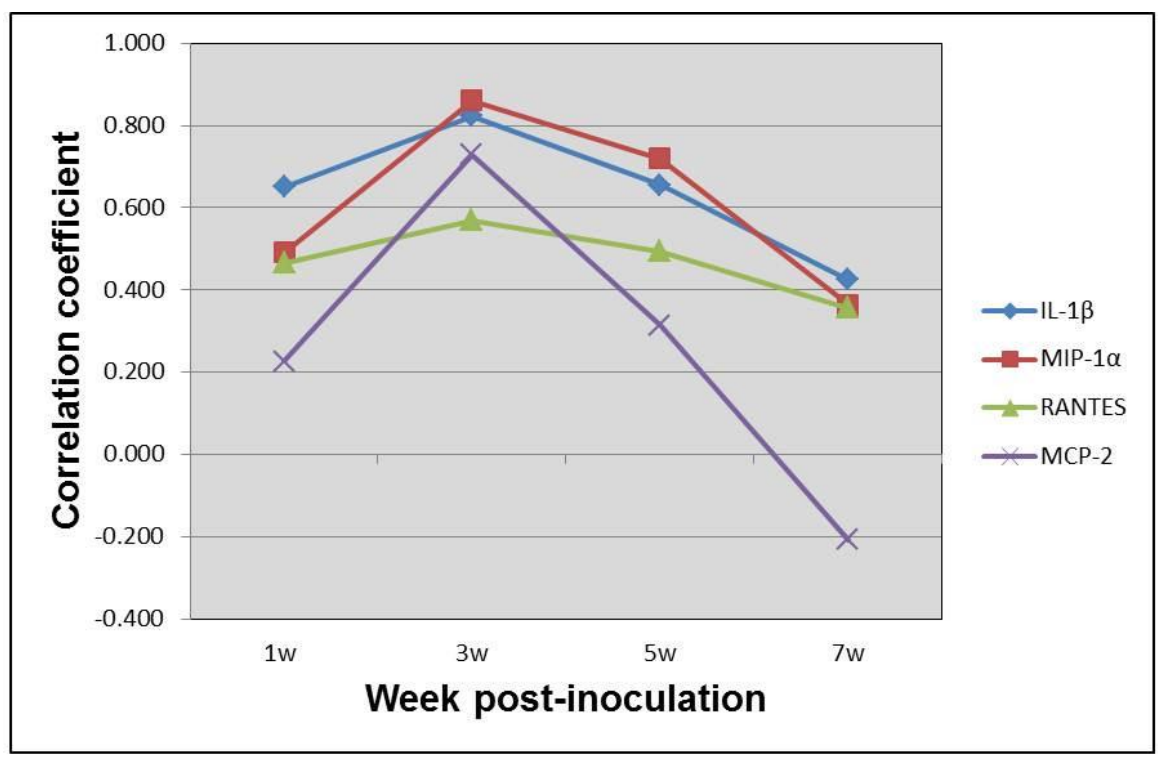

Figure 3.8 Correlation coefficient $\left(R^{2}\right.$ value) between normalized expression of IL-1 $\beta$ (blue line), MIP-1 $\alpha$ (red line), RANTES (green line), and MCP-2 (purple line) in $\mathrm{H}$. bilis-infected Smad3 ${ }^{-1-}$ mice $(n=14)$ from 1 to 7 weeks post-inoculation (PI) and lesion severity at 9 weeks PI as determined by histological analysis by two blinded pathologists; Spearman's rank order correlation. 

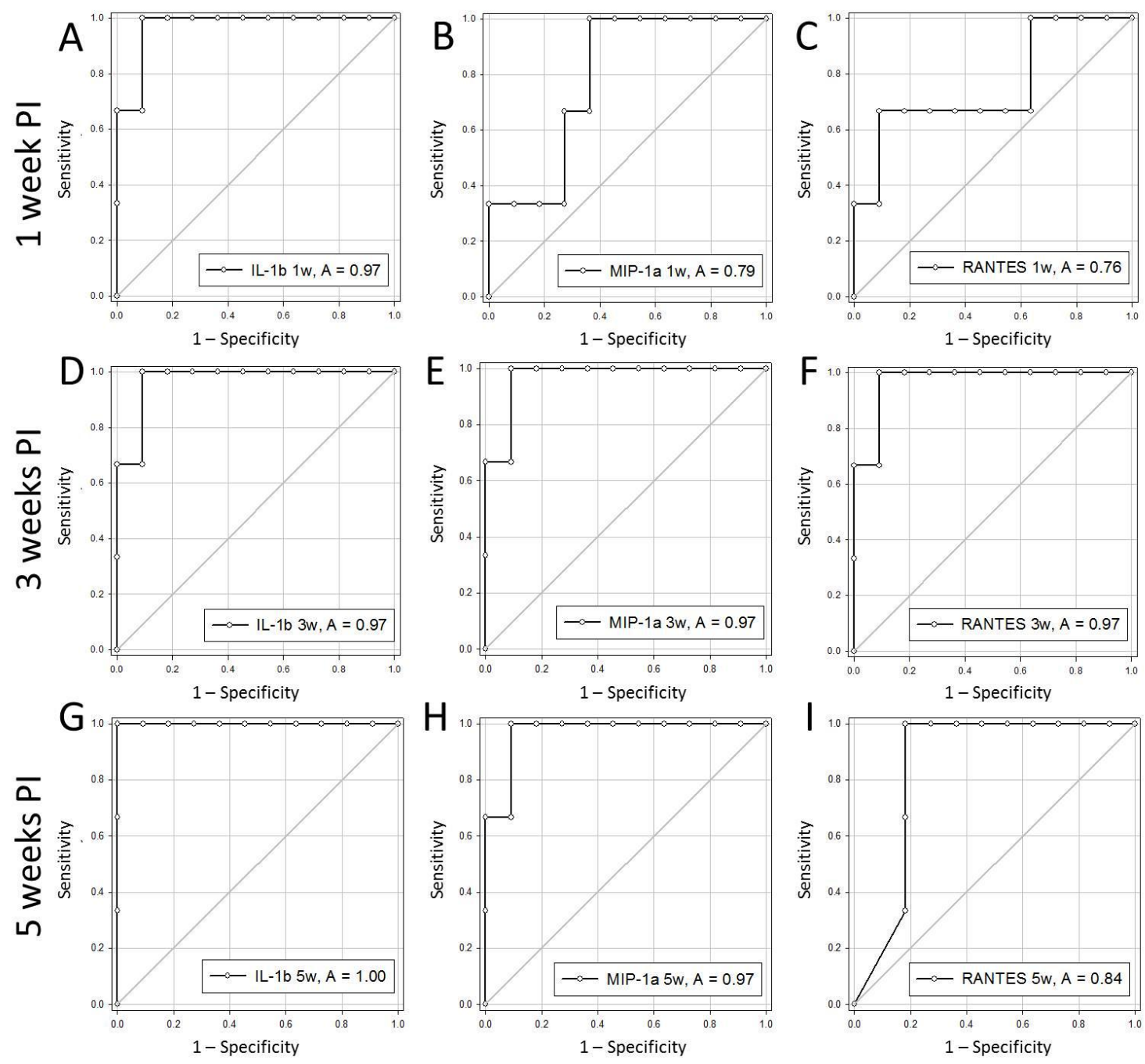

Figure 3.9 Receiver operating characteristic curves for mRNA levels of IL-1 $(A, D, G), M I P-1 \alpha(B$, $E, H)$, and $\operatorname{RANTES}(C, F, I)$ at one week $(A, B, C)$, three weeks $(D, E, F)$, and five weeks $(G, H, I)$ post-inoculation with $H$. bilis in the feces of Smad3 $\%$ mice $(n=14)$. 


\begin{tabular}{|c|c|c|}
\hline \multirow[t]{2}{*}{ Gene } & \multicolumn{2}{|r|}{ Antisense $\left(3^{\prime}-5^{\prime}\right)$} \\
\hline & Fragment size (bp) & Annealing temp. $\left({ }^{\circ} \mathrm{C}\right)$ \\
\hline \multirow[t]{2}{*}{ HPRT } & \multicolumn{2}{|c|}{ GTAATGATCAGTCAACGGGGGAC CCAGCAAGCTTGCAACCTTAACA } \\
\hline & 177 & 60 \\
\hline \multirow[t]{2}{*}{ 18s rRNA } & \multicolumn{2}{|c|}{ GAACGTCTGCCCTATCAAC CCTCGAAAGAGTCCTGTATTG } \\
\hline & 201 & 58 \\
\hline \multirow[t]{2}{*}{ IL-1 $\beta$} & AGCCCATCCTCTGTGACTCATG & GCTGATGTACCAGTTGGGGAAC \\
\hline & 420 & 57 \\
\hline \multirow[t]{2}{*}{ MIP-1 $1 \alpha$} & GCTCAACATCATGAAGGTCTCC & TGCCGGTTTCTCTTAGTCAGG \\
\hline & 222 & 57 \\
\hline \multirow[t]{2}{*}{ RANTES } & GAGTATTTCTACACCAGCAGC & GGACTAGAGCAAGCAATGC \\
\hline & 192 & 60 \\
\hline MCP-2 & \multicolumn{2}{|c|}{ ACTAAAGCTGAAGATCCCCCTTCGACATCACCTGCTTGGTCTGGAAAA } \\
\hline & 100 & 57 \\
\hline
\end{tabular}

Table 1. Sense and antisense primers, expected product sizes, and annealing temperatures used for real-time polymerase chain reaction amplification 


\section{CHAPTER 4}

\section{BACKGROUND}

Murine Norovirus (MNV) is a recently discovered, non-enveloped virus with a linear, positive-sense, ssRNA genome, of the family Caliciviridae. In humans, noroviruses are considered the primary cause of non-bacterial gastroenteritis, and are frequently foodborne or waterborne pathogens ${ }^{289-291}$. Both human and murine norovirus are relatively stable in the environment, however MNV was the first norovirus capable of cultivation, offering a useful tool for the study of human norovirus ${ }^{292}$. Since this time, multiple diagnostic assays have been developed for the detection of MNV in mice including serologic and PCR-based assays ${ }^{293}$.

A recent epidemiologic study detected antibodies against MNV1 in $22.1 \%$ of serum samples submitted from research facilities in North America making MNV the most common viral pathogen in research mouse populations ${ }^{293}$. Despite the fact that MNV infection is typically subclinical, there is reasonable concern that MNV may be able to cause variation in mouse models of IBD such as DSS- or TNBS-induced colitis, for several reasons. First, like human noroviruses, MNV resides in enteric tissues with the highest levels detected in the small intestines. Second, the principal cellular targets of MNV within the gastrointestinal tract are macrophages and dendritic cells ${ }^{294}$. Antigenpresenting cells play important roles in most murine models of IBD, and viral infection may modulate their function in multiple ways.

As intracellular microbes, viruses often induce an adaptive immune response characterized by IFN- $\gamma$-producing $\mathrm{T}_{\mathrm{H}} 1 \mathrm{CD} 4^{+} \mathrm{T}$ helper cells, the development of which is 
dependent on IL-12 produced by dendritic cells and macrophages. For example, in serum from humans experimentally infected with Snow Mountain Virus, a genogroup 2 norovirus, or treated with Norwalk virus-like particles, significantly elevated levels of IFN- $\gamma$ indicate an ongoing $\mathrm{T}_{\mathrm{H}} 1$ response ${ }^{295,296}$. Acute MNV infection may lead to a similar upregulation of IL-12 and IFN- $\gamma$, and thus enhance the inflammation seen in $\mathrm{T}_{\mathrm{H}} 1$ dependent IBD models such as TNBS-induced colitis. Additionally, MNV has been associated with an enhanced inflammatory response in one model of IBD with a concurrent increase in the number of splenic CD11 $\mathrm{c}^{+}$cells (dendritic cells).

Alternatively, in models such as DSS-induced colitis, in which the inflammation is due to disruption of the epithelial barrier and increased permeability of epithelial tight junctions, antigen presenting cells (APCs) have been shown to have a protective effect. Mice experimentally depleted of macrophages and dendritic cells developed a more severe colitis following administration of DSS ${ }^{297,298}$. This is thought to be due to a loss of two APC functions: the regulation of neutrophil infiltration and activation, and the clearance of DSS from the gut ${ }^{297}$. Thus, if infection with MNV adversely affects these APC functions, the inflammation induced by DSS may be exacerbated.

Herein, we examined the effect of acute experimental MNV infection on both DSSinduced, and TNBS-induced colitis, two commonly used mouse models of IBD characterized by acute inflammation. Following the onset of administration of either compound, clinical signs and histopathological evidence of disease typically manifest within days ${ }^{299}$. Using the routine outcomes measures of mortality, weight loss, and histological lesions, we were unable to detect an effect of MNV infection in either model. 


\section{MATERIALS AND METHODS}

Animals. Five to six week old female SJL/J, BALB/cJ, and C57BL/6J mice (Jackson Laboratories, Bar Harbor, ME, USA) were group-housed in separate polycarbonate microisolator cages with filter tops, in a cubicle under negative pressure with a controlled environment of $21 \pm 2^{\circ} \mathrm{C}, 40-60 \%$ humidity, 15 air changes per hour, and $12: 12 \mathrm{~h}$ light:dark cycle. Mice received pelleted mouse chow (Purina 5008 Formulab Diet; Purina Mills Inc., Richmond, IN, USA) and acidified water ad libitum. Mice were allowed a minimum of 3 days for acclimation prior to any experimental manipulation. Mice were purchased from a colony that is historically free of common murine pathogens including MHV, MVM, MPV, Sendai, Mycoplasma pulmonis, TMEV, EDIM, PVM, Reo3, LCMV, Ectromelia, and Helicobacter spp. Upon arrival, mice were confirmed to be negative for MNV by fecal PCR, performed at the Research Animal Diagnostic Laboratory (RADIL). MNV infection was confirmed in the experimental group via PCR of feces collected at necropsy. Housing and all procedures involving animals were performed according to a protocol approved by the University of Missouri Institutional Animal Care and Use Committee, and in compliance with the Animal Welfare Act and The Guide for The Care and Use of Laboratory Animals.

Viral Propagation and Concentration. MNV-4 was initially isolated from the mesenteric lymph nodes (MLN) of naturally-infected mice of various strains and stocks, positive for antibodies against MNV-4 by microbead fluorescent immunoassay as

previously described ${ }^{293}$. MLN were pooled in groups of 15 to 20 in $4 \mathrm{ml}$ Dulbecco's modified Eagle's medium (Hyclone, Logan, UT) supplemented with $10 \%$ low-endotoxin fetal bovine serum (Cambrex, East Rutherford, NJ), $10 \mathrm{mM}$ 4-(2-hydroxyethyl)-1- 
piperazineethanesulfonic acid, and $10 \mu \mathrm{g} / \mathrm{ml}$ ciprofloxacin (supplemented growth medium). Pooled samples were processed in a blender (Stomacher 80 Lab Blender, Tekmar Company, Cincinnati, OH) and clarified by centrifugation for $10 \mathrm{~min}$. at $10,000 \times$ g. RAW 264.7 cells were grown to $80 \%$ confluence in $25-\mathrm{cm}^{2}$ cell culture flasks, and $1 \mathrm{ml}$ clarified MLN homogenate was inoculated onto RAW cells and incubated for 1 $\mathrm{h}$ at $37^{\circ} \mathrm{C}$ and $5 \% \mathrm{CO}_{2}$, after which the inoculum was replaced with fresh supplemented growth medium and incubated for $1 \mathrm{~h}$ at $37^{\circ} \mathrm{C}$ and $5 \% \mathrm{CO}_{2}$. Cultures were monitored visually, and cells displaying cytopathic effect (CPE), suggestive of MNV infection, were pelleted at $2000 \times \mathrm{g}$ for $10 \mathrm{~min}$. at $4^{\circ} \mathrm{C}$, and the clarified supernatants containing virus were frozen at $-80^{\circ} \mathrm{C}$. Sham inoculations were similarly prepared RAW 264.7 cell cultures without added MNV.

Plaque Assay. The plaque assay was adapted from one previously described (Bac-NBlue transfection kit, Invitrogen, Carlsbad, CA). Briefly, RAW 264.7 cells were seeded onto $60-\mathrm{mm}$ diameter tissue culture plates at a density of $5 \times 10^{6}$ cells and allowed to adhere for $5 \mathrm{~h}$ at $37^{\circ} \mathrm{C}$. Tenfold serial dilutions of MNV-4 were made on ice using supplemented DMEM and inoculated in duplicate onto culture plates containing a confluent layer of RAW cells. After inoculation for $1 \mathrm{~h}$ at $37^{\circ} \mathrm{C}$, the inoculum was aspirated and replaced with a mixture of one part $2.5 \%$ SeaPlaque agarose and three parts supplemented DMEM, allowed to solidify, and incubated at $37^{\circ} \mathrm{C}$ for 24 to $48 \mathrm{~h}$ until plaques were visible. For better visualization of the plaques, neutral red $(0.01 \%$ final concentration) was added to $3 \mathrm{ml}$ of the mixture containing $2.5 \%$ SeaPlaque agarose and supplemented DMEM, allowed to solidify, and incubated at $37^{\circ} \mathrm{C}$ for 6 to $8 \mathrm{~h}$. 
Infection with MNV-4. Mice were inoculated via gastric gavage with approximately 0.2 $\mathrm{mL}$ of clarified supernatant from MNV-4-infected RAW264.7 cell culture, containing approximately $1 \times 10^{7} \mathrm{PFU}$ of MNV-4. Control mice were gavaged with an equivalent volume of clarified supernatant from an uninfected RAW264.7 cell culture. Mice were group-housed according to MNV infection status prior to induction of colitis. Both models of colitis were induced four days after infection.

Induction of Experimental Colitis - DSS. Dextran sulfate sodium (DSS: average molecular weight 36,000-50,000) was purchased from MP Biomedicals, LLC (Solon, $\mathrm{OH}$, USA). Experimental colitis was induced by giving drinking water containing 3.5\% or $5.0 \%(\mathrm{wt} / \mathrm{vol}) \mathrm{DSS}$ to C57BL/6J and BALB/cJ mice $(n=10)$, respectively for 5 days (changed daily), followed by normal drinking water for 5 days. Mice were then sacrificed via an overdose of inhaled $\mathrm{CO}_{2}$ and the entire GI tract was collected for histopathological evaluation.

Induction of Experimental Colitis - TNBS. Trinitrobenzenesulfonic acid (TNBS) was purchased from Spectrum Chemical Mfg. Corp. (Gardena, CA, USA). Mice were anesthetized via inhaled isoflurane, and either 1.0 or $2.5 \mathrm{mg}$ TNBS in $150 \mu \mathrm{l}$ of $50 \%$ ethanol was instilled per rectum using a $22 \mathrm{~g}$ IV catheter $(n=40)$. Mice were then suspended by the tail base for 20-30 seconds to minimize expulsion of the TNBS. Mice were recovered from anesthesia in a cage lined with an absorbent material, as they often expelled a small portion of the TNBS upon recovery. Four or ten days later $(n=20$ per time point), mice were sacrificed via an overdose of inhaled $\mathrm{CO}_{2}$ and the entire GI tract was collected for histopathological evaluation. 
Clinical Evaluation of Colitis. Following induction of colitis, mice were weighed daily and evaluated for signs of illness, including hunched posture, rough haircoat, or lethargy. The presence of any of the above criteria was considered a clinical indication of disease. Mortality was also recorded as binary nominal data.

Lesion Scores. Gastrointestinal tracts were prepared as gut rolls and examined by two pathologists in a blinded fashion. Briefly, for TNBS- and DSS-induced colitis, lesion severity was ranked based on the number and longitudinal extent of cecal and colonic erosions and ulcerations ( 0 to 3 ), the severity of inflammation (0 to 3 ), and in the case of DSS, evidence of hyperplasia (0 to 3 ), or in the case of TNBS, evidence of peritonitis (0 to 3), Scores from the three categories were then added to yield a total histological lesion score ranging from zero to a maximum of nine. Slides were then placed in order of their numeric lesion scores and compared, again in a blinded fashion, and ranked in order of severity. Comparisons between MNV-infected and sham-inoculated mice was performed using ANOVA on ranks. 


\section{RESULTS}

DSS Colitis. Previous studies have demonstrated differential susceptibility of various mouse strains to DSS-induced colitis. While both $\mathrm{C} 57 \mathrm{BL} / 6 \mathrm{~J}$ and $\mathrm{BALB} / \mathrm{cJ}$ mice are considered susceptible, C57BL/6J mice require lower levels of DSS in the drinking water to avoid excessive mortality. In addition, several differences have been demonstrated in the metabolic capacity of macrophages from these two mouse strains to respond to microbes $^{300,301}$. Thus, considering the protective role of dendritic cells and macrophages in this model of colitis, we analyzed the effect of MNV in both strains. Following administration of $3.5 \%$ or $5 \%$ DSS in the drinking water of C57BL/6J and BALB/cJ mice respectively, clinical signs were very subtle, and there was no mortality throughout the experiment in either strain of mice, independent of infection status. Additionally, the mice were weighed daily and their weight normalized to their weights prior to induction of colitis. The weight of the mice in the MNV-infected and sham-inoculated groups did not differ significantly (figure 4.1) in either mouse strain.

Following administration of DSS for consecutive five days followed by five days without DSS, mice were euthanized and their entire GIT from jejunem to rectum was collected for histological analysis, which revealed a loss of crypt architecture, mild to moderate inflammation, (figure 4.2) in the majority of mice, and occasional ulceration in a few. Lesions were confined to the colon and, to a lesser degree, the cecum. A total lesion score was generated for each mouse based on the degree of ulceration, inflammation, and hyperplasia. While there was a trend toward higher lesion scores in the C57BL/6J mice when compared to the BALB/cJ, there was no significant difference in the lesion scores of MNV-infected and sham-inoculated mice, in either strain (figure 4.3). 
TNBS Colitis. As with DSS-induced colitis, one group of mice was orally gavaged with MNV-4 four days prior to induction of colitis, while the control group was sham inoculated with an equivalent volume of RAW 264.7 culture supernatant. A dosage of $2.5 \mathrm{mg}$ of TNBS per mouse was used for all experiments as preliminary studies showed that this produced more consistent inflammation in SJL/J mice than did $1.0 \mathrm{mg}$ TNBS. Following administration of TNBS, mice were weighed daily and their weight was normalized to their weight at day 0. As in DSS-induced colitis, there was no significant difference in weight loss between the two groups (figure 4.4). Clinical signs and mortality were sporadic in both groups however and no differences in survival were detected between MNV-infected and sham-inoculated mice (figure 4.5). Considering the acute nature of TNBS-induced colitis, samples were collected at both day 4 and day 10 , for histopathological analysis and lesion scoring. Lesions included focal and multifocal areas of ulceration and inflammation characterized by infiltrates of mononuclear cells and neutrophils (figures 4.6A and B). Several mice had areas of transmural inflammation resulting in inflammatory infiltrates reaching the peritoneal cavity (figures 4.6C and D). Lesion scores were higher at the 4 day time point than at day 10 in both groups but again, there was no difference detected between the two groups at either time point (figure 4.7). 


\section{DISCUSSION}

Following the recent discovery of MNV, several groups began to question its ability to induce inflammation in various mouse strains, or affect the outcome of mouse models of disease. Despite the ability of MNV to rapidly disseminate to multiple tissues in immunocompetent mice ${ }^{302}$, it appears to be subclinical in all immunocompetent strains and stocks examined to date, including 129S6/SvEvTac, C57BL/6, Tac:SW, Hsd:ICR mice $^{293,303,304}$. Similarly, histological lesions are not seen in naturally infected immunocompetent mice and even acute experimentally-induced lesions are extremely mild and transient ${ }^{302}$. Highlighting the importance of the innate immune system in response to MNV infection, $\mathrm{Rag}^{-/-}$mice lacking functional $\mathrm{T}$ and $\mathrm{B}$ cell receptors do not develop clinical signs or histological lesions while mice deficient for STAT1 or IFN $\alpha \beta \gamma$ R rapidly succumb to MNV infection due to hepatic and pulmonary inflammation ${ }^{302,305}$. Thus it appears that an intact IFN signaling pathway is essential for prevention of MNVinduced lesions. While MNV does not induce de novo lesions in immunocompetent mice, it is possible however that MNV may alter experimentally induced inflammation. Considering the anatomical and cellular tropisms of MNV, we studied two commonly used mouse models of IBD, DSS- and TNBS-induced colitis.

In humans, several studies have attempted to link persistent or prior viral infection, as well as vaccination against, or in utero exposure to, a viral pathogen with subsequent

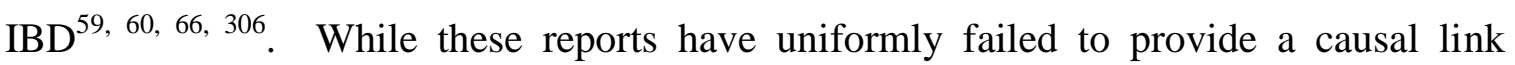
between viral infection and subsequent $\mathrm{IBD}^{307}$, some have demonstrated an increased relative risk of developing IBD later in life following exposure to certain viruses ${ }^{308,} 309$. Regarding murine models of IBD, it is unknown whether typically subclinical enteric 
viral infection may alter the disease phenotype. $\mathrm{MNV}$ is a highly prevalent, enteric virus of mice that resides in APCs making it a potential confounding factor in IBD research using mouse models.

We chose to examine the effect of MNV on the DSS and TNBS colitis models based on their popularity in the IBD research community, as well as their complementary disease mechanisms ${ }^{299}$. While TNBS is generally accepted to be mediated by a combined $\mathrm{T}_{\mathrm{H}} 1 / \mathrm{T}_{\mathrm{H}} 17$ adaptive immune response, DSS colitis is driven by an innate response following disruption of the epithelial barrier and crypt loss. Additionally, we chose to include both C57BL/6 and BALB/c mouse strains in our DSS studies based on reported differences in the metabolic capacity of macrophages.

Our data support the notion that $\mathrm{MNV}$ is unlikely to significantly alter the disease phenotype in these models of IBD, at least with regard to the commonly measured outcomes such as mortality and histologic evidence of inflammation. In our studies, there was no difference in weight loss, clinical signs, mortality, or severity of histological lesions in either DSS- or TNBS-induced colitis. It should be remembered however that this does not preclude MNV from affecting other models of IBD. Chase et al. demonstrated that infection with MNV affects weight loss and histological disease severity in Helicobacter bilis-infected MDR $1 \mathrm{a}^{-/-}$mice $\mathrm{e}^{310}$. Thus, as different models of IBD have different mechanisms and can sometimes be performed on more than one strain of mouse, the relevance of MNV infection in experimental subjects must be considered on an individual basis. In addition, we measured clinical signs including weight and histological lesions. While we were unable to detect differences in these endpoints, that 
is clearly not to say that infection with MNV does not cause perturbations in cytokine expression and other parameters. 


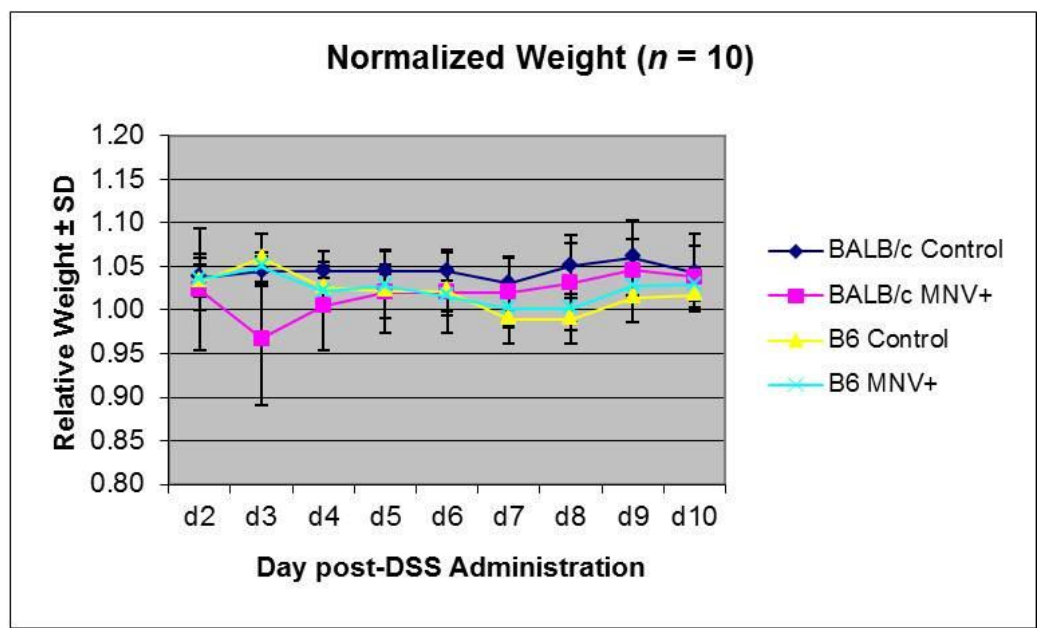

Figure 4.1 Weight of MNV- and sham-inoculated $\mathrm{BALB} / \mathrm{c}$ and C57BL/6 (B6) mice $(n=10)$ normalized to their weight prior to induction of colitis with DSS \pm standard deviation. Statistically significant $(p<0.05)$ differences are denoted by an asterisk; repeated measures ANOVA. 


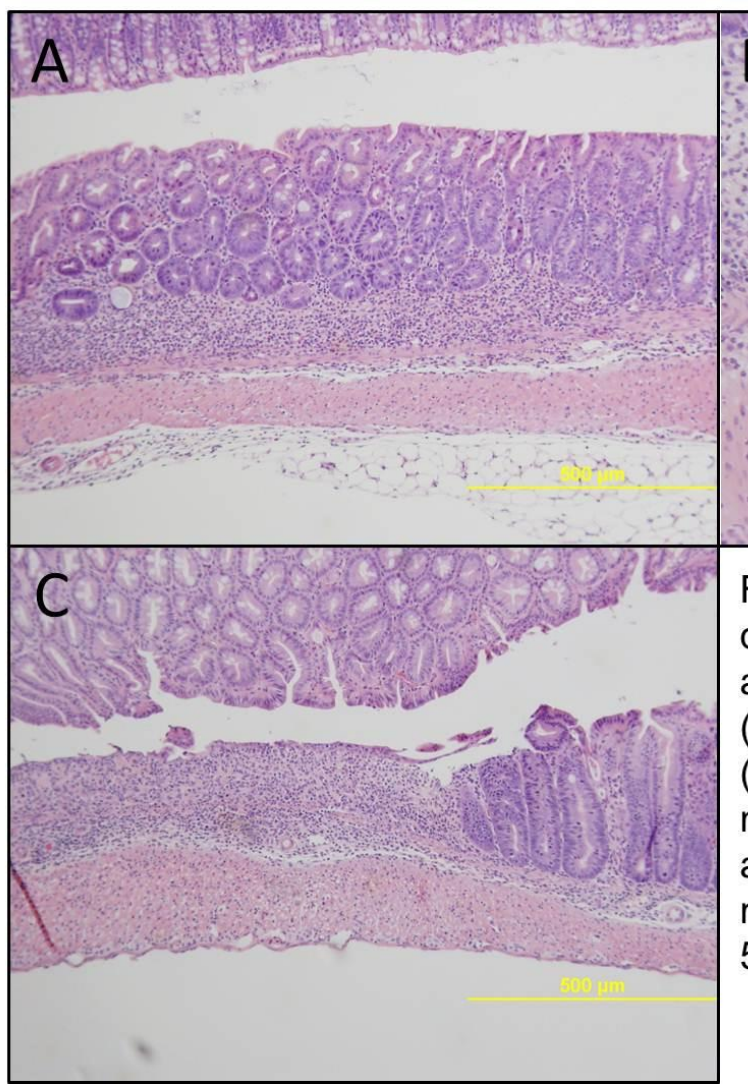

Figure 4.2 Representative images demonstrating the typical histological appearance of inflammatory infiltrates (A, B) and ulcerations and hyperplasia (C) in the colon in DSS-treated C57BL/6 mice after five days of DSS administration followed by five days of no DSS; Hematoxylin and eosin stain at $50 \times(A, C)$ and $100 \times(B)$ magnification. 


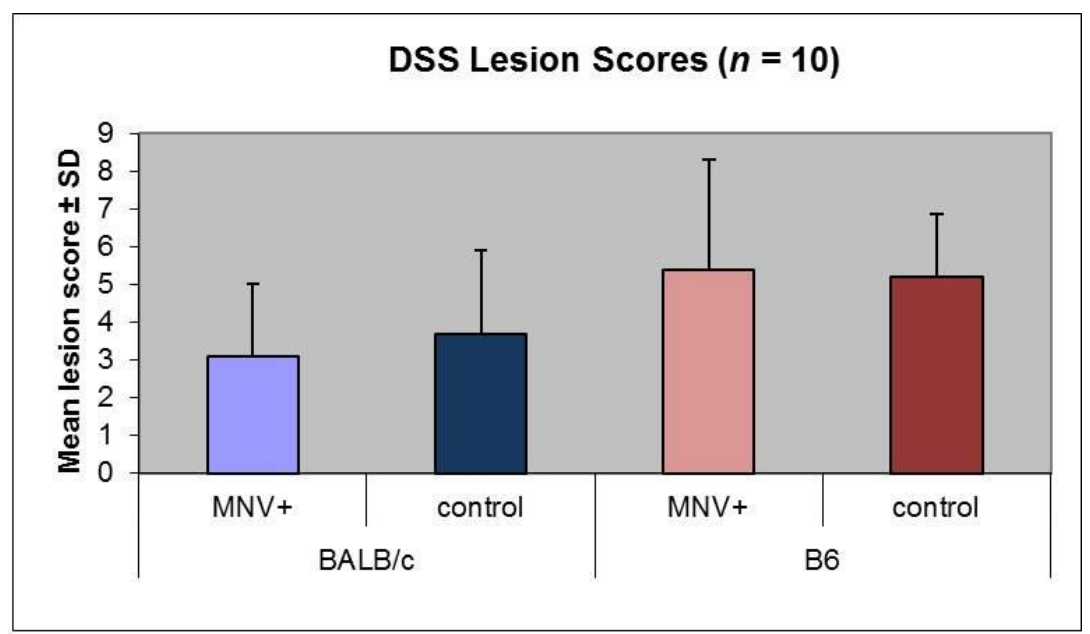

Figure 4.3 Lesion scores from MNV- and sham-infected $\mathrm{BALB} / \mathrm{c}$ and $\mathrm{C} 57 \mathrm{BL} / 6$ (B6) mice given $5 \%$ and $3.5 \%$ DSS respectively after five days of DSS administration followed by five days of no DSS. Bars represent mean lesion score \pm standard deviation. Significantly different $(p<0.05)$ differences are denoted by an asterisk; student's t-test. 


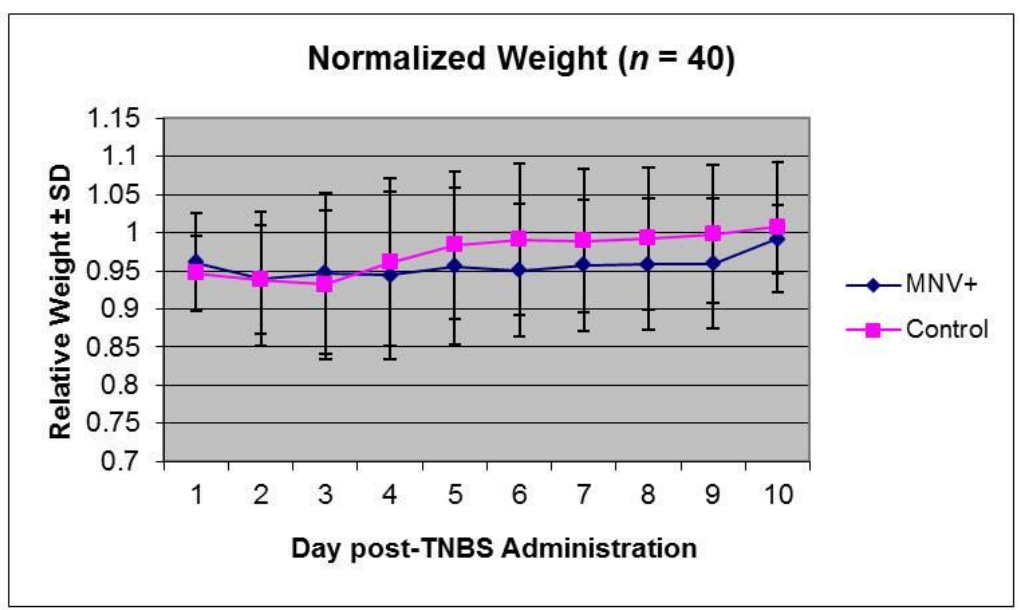

Figure 4.4 Weight of MNV- and sham-inoculated SJL/J mice $(n=40)$ normalized to their weight prior to induction of colitis with TNBS \pm standard deviation. Statistically significant $(p<0.05)$ differences are denoted by an asterisk; repeated measures ANOVA. 


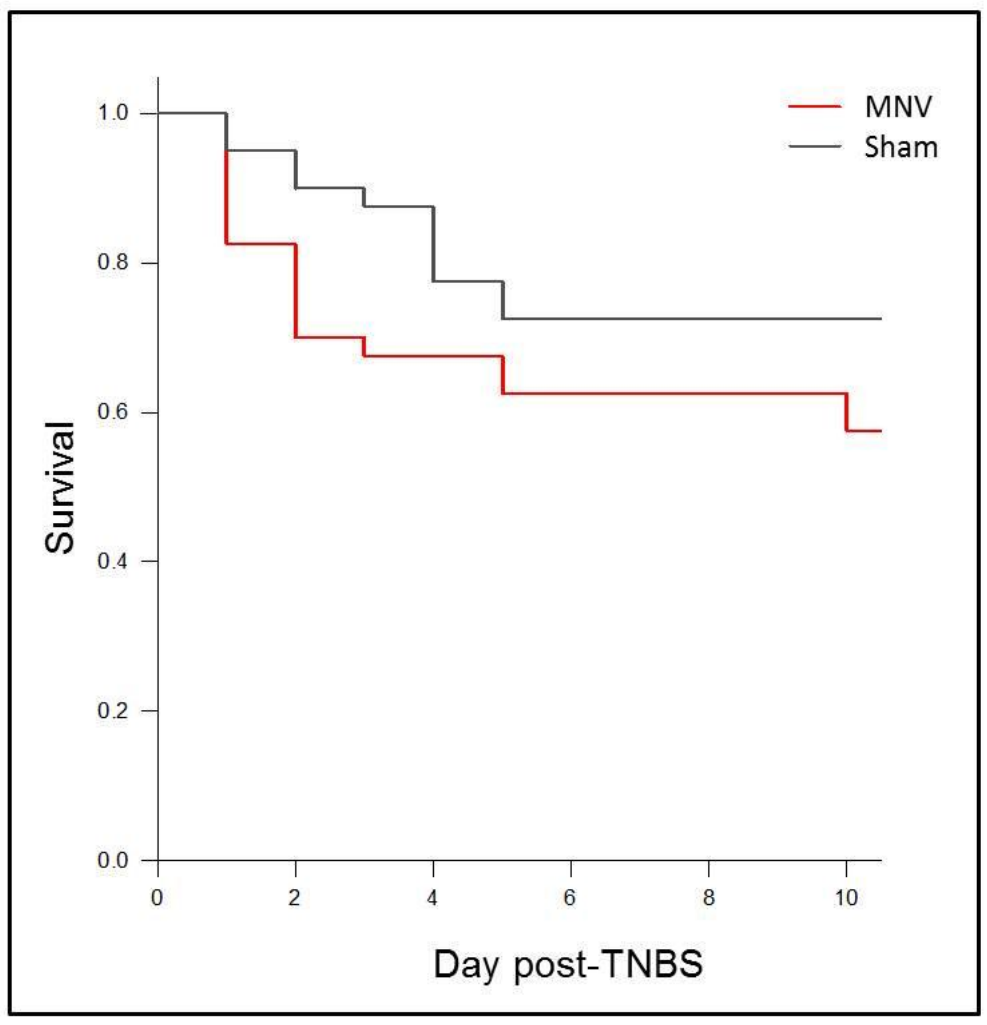

Figure 4.5 Kaplan-Meier survival curve showing mortality in MNV- and sham-inoculated SJL/J mice ( $n$ $=40$ ) following rectal instillation of $2.5 \mathrm{mg}$ TNBS . Statistically significant $(p<0.05)$ differences are denoted by an asterisk; Kaplan-Meier log-rank survival analysis. 


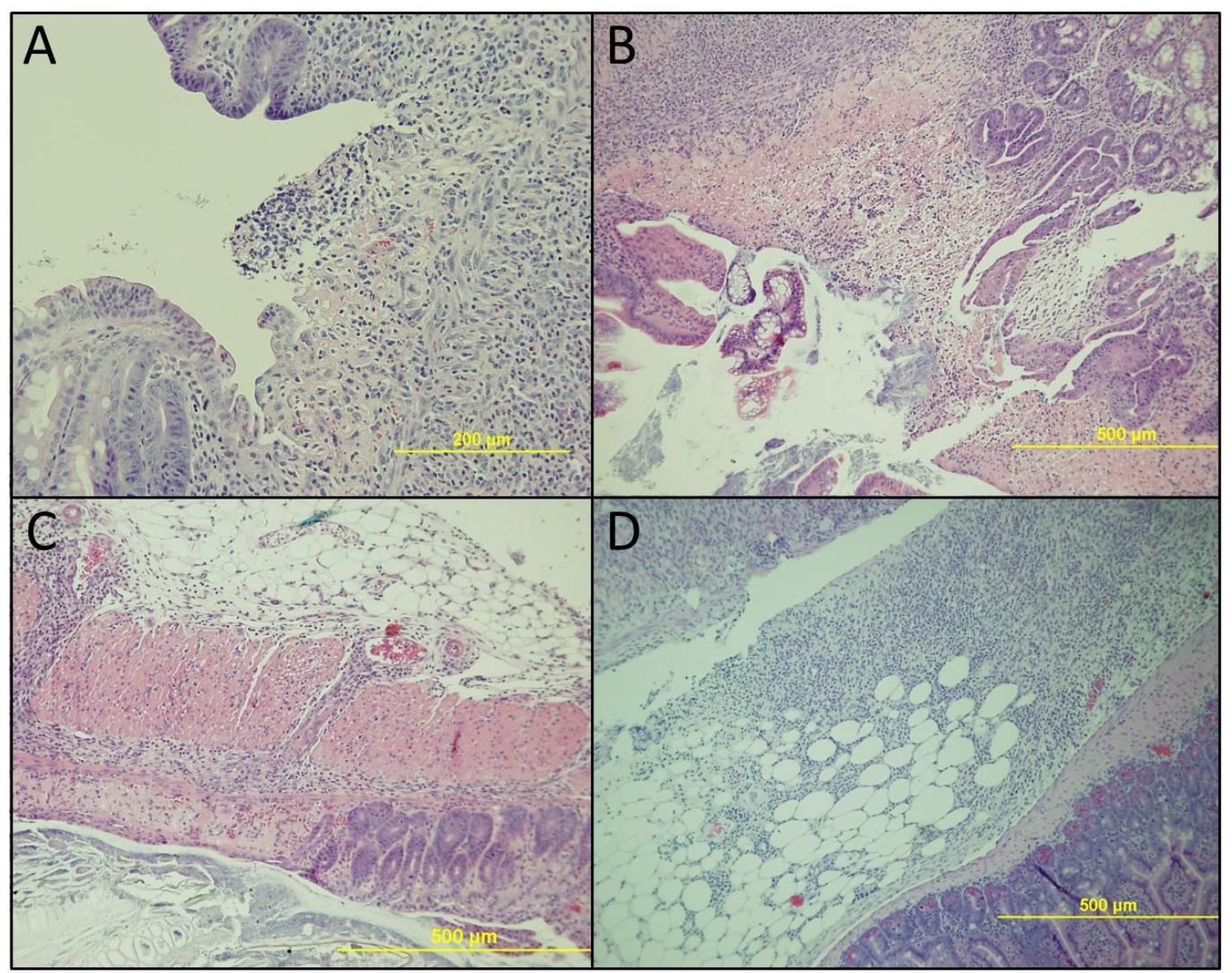

Figure 4.6 Representative images demonstrating the typical histological appearance of inflammatory infiltrates (A, B), ulceration (C), and peritonitis (D) in the colon in TNBStreated SJL/J mice ten days after TNBS administration. Hematoxylin and eosin stain at $100 \times(A)$ and $50 \times(B, C, D)$ magnification. 


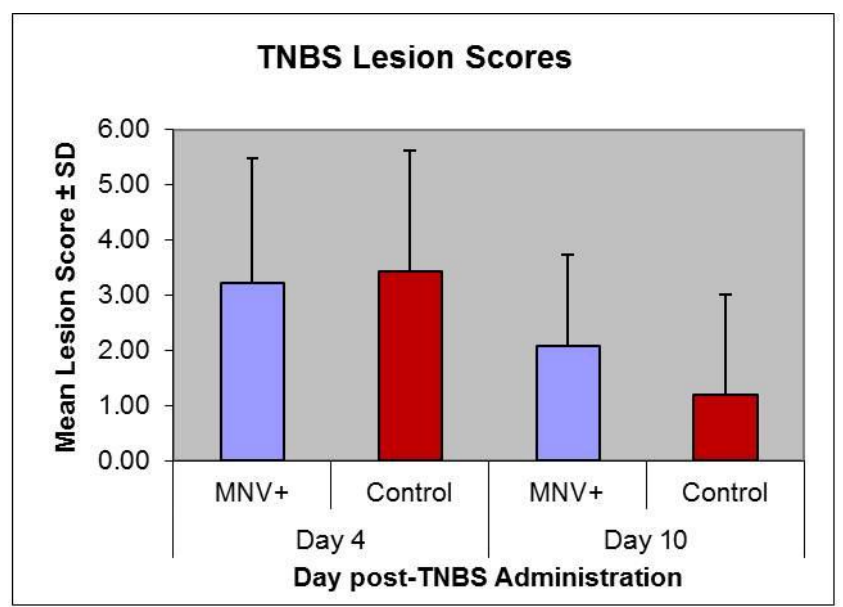

Figure 4.7 Lesion scores from MNV- and shaminfected SJL/J mice four $(n=20)$ or ten $(n=40)$ days after rectal instillation of $2.5 \mathrm{mg}$ TNBS. Bars represent mean lesion score \pm standard deviation. Significantly different $(p<0.05)$ differences are denoted by an asterisk; student's t-test. 


\section{CHAPTER 5}

Comparative medicine is founded on the concept that through the use of appropriate animal models of disease, knowledge can be gained regarding the human condition, with the ultimate goal of translating that knowledge into useful therapeutic and diagnostic modalities. In the studies described herein, Helicobacter-induced mouse models of inflammatory bowel disease and colitis-associated colorectal cancer were chosen due to their phenotypic similarities to the human diseases, but also due to the fact that the inflammation in these models is putatively driven by not just $H$. hepaticus and $H$. bilis, but also the commensal intestinal microbiota, as is believed to occur in IBD. The studies described in chapters 2 and 3 generated several exciting findings, each with the potential to be directly translated to human gastroenterology and oncology.

In chapter 2, we generated several pieces of evidence supporting the hypothesis that CD $8 \alpha^{+}$dendritic cells DC) are involved in the initiation of an inflammatory cascade which takes months to manifest. Of note, we determined that while multiple DC subsets are present in significantly greater numbers in naïve mice which considered susceptible to H. hepaticus-induced cecal inflammation, the $\mathrm{CD}^{2} \alpha^{+} \mathrm{CD} 11 \mathrm{~b}^{-}$and $\mathrm{CD} 8 \alpha^{-} \mathrm{CD} 11 \mathrm{~b}^{-}$(double negative, DN) subsets are preferentially recruited to the cecum following inoculation with H. hepaticus. Additionally, while cells from the susceptible mouse strain produce greater amounts of IL-12/23p40 than cells from a resistant mouse strain when naïve mice are used, cells from the susceptible mouse strain acutely infected with $H$. hepaticus produce significantly greater amounts of TNF- $\alpha$, CXCL10 (IP-10), and CCL5 (RANTES). While previous studies had demonstrated increased transcription of several of these factors in 
susceptible mice, confirmation that these inflammatory mediators are expressed at a significantly greater level at the protein level is an essential step in selecting molecules as therapeutic targets. Current biologic therapies targeting TNF- $\alpha$ are currently available for IBD patients, lending additional support for the use of $H$. hepaticus-infected A/J mice as a model of IBD. However, due to the heterogeneous nature of IBD, not all patients respond to anti-TNF- $\alpha$ therapy, suggesting that other molecules, both immunologic and otherwise, are needed. Chemokines offer one possibility for therapeutic targets. Perhaps by blocking the homing of inflammatory cells back to the GIT, disease can be abrogated.

Of particular interest is the data supporting a role for $\mathrm{CD} 8 \alpha^{+} \mathrm{DC}$ in disease initiation. Future studies will confirm the physiological role of this subset of DC in the pathogenesis of $H$. hepaticus-induced inflammation. We plan to inoculate wild type and Batf3deficient $\mathrm{A} / \mathrm{J}$ mice with $H$. hepaticus, and then collect intestinal tissue from these mice at acute and chronic time points, to determine if the absence of $\mathrm{CD} 8 \alpha^{+} \mathrm{DC}$ is associated with a decrease or complete lack of inflammation. If the in vivo data confirm our hypothesis, this will provide some of the most compelling evidence of a role for a specific subset of DC in the pathogenesis of IBD. While this discovery would advance our overall understanding of IBD, it would be particularly exciting in light of recent developments in the field of gene therapy. Novel chemical conjugates have increased the cell specificity of gene delivery ${ }^{311}$, diminishing potential side effects. The ability to selectively deliver gene therapy to a specific subset of DC would therefore enhance the safety of these treatments. Of note, gene therapy augmenting PPAR $\gamma$ was recently shown to ameliorate inflammation in DSS-induced colitis ${ }^{312}$. 
We have also begun co-infection studies examining the effect of segmented filamentous bacteria (SFB) on the $H$. hepaticus-infected $\mathrm{A} / \mathrm{J}$ model. $\mathrm{SFB}$ has a role in the development of both regulatory $\mathrm{T}$ cells $\mathrm{s}^{313}$ and $\mathrm{T}_{\mathrm{H}} 17 \mathrm{~T}$ cells ${ }^{314}$. Accordingly, these bacteria are thought to play a large role in shaping the gut immune response as well as the composition of the microbiota. These studies will also incorporate analyses of the microbiota using multiple techniques including automated ribosomal intergenic spacer analysis (ARISA) and deep sequencing of the 16s rRNA gene.

The data from chapter 3 portend novel methods of screening IBD patients for dysplasia, an early stage of colitis-associated colorectal cancer (CAC). In H. bilis-infected Smad3 ${ }^{-/}$ mice, we were able to reliably detect early stages of disease using fecal mRNA-based biomarkers. Additionally, the expression of certain inflammatory mediators early after inoculation with $H$. bilis correlated with disease severity several weeks later, thus serving as a prognostic indicator. These findings are extremely exciting considering the weaknesses of current screening techniques for CAC in humans. Inexpensive and noninvasive techniques such as fecal occult blood tests suffer from very poor specificity. Thus many patients will undergo unnecessary confirmatory colonoscopy. Colonoscopy in itself offers relatively decent accuracy in tumors of the distal colon, however masses in the proximal colon are often inaccessible. Also of concern is the unpleasant and invasive nature of colonoscopy, attributes cited by IBD patients as reasons for noncompliance with the screening recommendations of the American Gastroenterological Association. Other noninvasive techniques such as virtual colonoscopy are expensive and require considerable expertise in their interpretation. Molecular techniques have the potential to provide inexpensive, noninvasive diagnoses capable of being performed with minimal 
special equipment. Additionally, methods using feces as the diagnostic sample could be performed with submitted samples, enhancing compliance by patients for whom travel to a clinic is problematic or impossible such as senior citizens, individuals living in rural communities, and deployed soldiers. There are currently molecular assays available for Lynch syndrome (hereditary nonpolyposis colorectal cancer) and familial adenomatous polyposis, the two recognized forms of hereditary colorectal cancer (CRC) which account for approximately 3 and $1 \%$ of CRC respectively. These assays utilize standard PCR methodology to identify polymorphic alleles of the genes associated with disease. While there are several genetic polymorphisms that have been associated with IBD, none have been identified in IBD patients as being responsible for an increased risk of developing CAC. There is evidence to support the notion that increased duration, severity, and longitudinal extent of inflammation in the colon increases the risk of CAC. Thus, fecal expression-based biomarkers using reverse-transcription PCR methodology may offer an effective means of monitoring the expression of various mediators noninvasively and affordably. Additionally, if specific biomarkers could be correlated with the very early stages of CAC development, i.e. dysplasia, disease could be treated more effectively and affordably.

To this end, we have established research team consisting of a biostatistician with experience in the analysis of large and complex biological data sets, and a consortium of gastroenterologists including clinicians from the University of Missouri, Washington University in St. Louis, the John Cochran VA Medical Center in St. Louis, and a local group of seven gastroenterologists in Columbia, all of whom perform routine annual colonoscopic screening of IBD patients for dysplasia. We're actively pursuing funding 
opportunities to translate the technique described in chapter 3 to an at-risk human population, namely IBD patients. Briefly, our experimental design is to obtain permission to have access to the results of colonoscopies, and then obtain a fecal sample from those patients in whom areas of dysplasia are identified (and an equivalent number of non-dysplastic IBD patients). RNA will be isolated from feces and the entire transcriptome screened using RNA-Seq technology $y^{315}$ to identify genes that are differentially transcribed between the two groups. Promising candidate genes will then be verified using individual RT-PCR reactions. Our goal is to identify a distinct expression-based signature correlating with dysplasia for use clinically as a screening method tailored to IBD patients. This technique has far-reaching implications in the diagnosis of other enteropathies and the studies described in chapter 3 provide proof-ofconcept. 


\section{BIBLIOGRAPHY}

1. Werts C, Rubino S, Ling A, et al. Nod-like receptors in intestinal homeostasis, inflammation, and cancer. J Leukoc Biol;90:471-82.

2. Molodecky NA, Soon IS, Rabi DM, et al. Increasing incidence and prevalence of the inflammatory bowel diseases with time, based on systematic review.

Gastroenterology; 142:46-54 e42; quiz e30.

3. Forbes A GE, Paulon E. Nutrition in inflammatory bowel disease. Journal of Parenteral and Enteral Nutrition 2011;35:571-580.

4. Gasche C, Lomer MC, Cavill I, et al. Iron, anaemia, and inflammatory bowel diseases. Gut 2004;53:1190-7.

5. Ulitsky A AA, Naik A, Skaros S, Zadvornova Y, Binion DG, Issa M. Vitamin D deficiency in patients with inflammatory bowel disease; association with disease activity and quality of life. Journal of Parenteral and Enteral Nutrition 2011;35:308-316.

6. Langholz E. Current trends in inflammatory bowel disease: the natural history. Therap Adv Gastroenterol;3:77-86.

7. Card T, Hubbard R, Logan RF. Mortality in inflammatory bowel disease: a population-based cohort study. Gastroenterology 2003;125:1583-90.

8. Chen KS, Reyes, E. B., Goldberg, G. A., Dubois, R. W. Costs associated with disease severity in six selected chronic diseases in managed care settings. 27th Annual Meeting of The Society for Medical Decision Making (October 21-24, 2005). San Francisco, CA, 2005. 
9. Bassi A, Dodd S, Williamson P, et al. Cost of illness of inflammatory bowel disease in the UK: a single centre retrospective study. Gut 2004;53:1471-8.

10. Juan J, Estiarte R, Colome E, et al. Burden of illness of Crohn's disease in Spain. Dig Liver Dis 2003;35:853-61.

11. Gibson $\mathrm{TB}, \mathrm{Ng}$ E, Ozminkowski RJ, et al. The direct and indirect cost burden of Crohn's disease and ulcerative colitis. J Occup Environ Med 2008;50:1261-72.

12. van der Valk ME, Mangen MJ, Leenders M, et al. Healthcare costs of inflammatory bowel disease have shifted from hospitalisation and surgery towards anti-TNFalpha therapy: results from the COIN study. Gut.

13. Bernstein CN, Longobardi T, Finlayson G, et al. Direct medical cost of managing IBD patients: a Canadian population-based study. Inflamm Bowel Dis;18:1498508.

14. Stark R, Konig HH, Leidl R. Costs of inflammatory bowel disease in Germany. Pharmacoeconomics 2006;24:797-814.

15. Blomqvist P, Ekbom A. Inflammatory bowel diseases: health care and costs in Sweden in 1994. Scand J Gastroenterol 1997;32:1134-9.

16. Nguyen GC, Tuskey A, Dassopoulos T, et al. Rising hospitalization rates for inflammatory bowel disease in the United States between 1998 and 2004. Inflamm Bowel Dis 2007;13:1529-35.

17. Earnshaw VA, Quinn DM. The impact of stigma in healthcare on people living with chronic illnesses. J Health Psychol;17:157-68. 
18. Mittermaier C, Dejaco C, Waldhoer T, et al. Impact of depressive mood on relapse in patients with inflammatory bowel disease: a prospective 18-month follow-up study. Psychosom Med 2004;66:79-84.

19. Yan J, Tamboli CP. Testing concordance of clinical characteristics in familial studies with application to inflammatory bowel diseases. Biom J 2007;49:840-53.

20. Palli D, Masala G, Saieva C. Population-based studies of IBD incidence in Italy and capture-recapture methods. Int J Epidemiol 1997;26:904-6.

21. Orholm M, Binder V, Sorensen TI, et al. Concordance of inflammatory bowel disease among Danish twins. Results of a nationwide study. Scand J Gastroenterol 2000;35:1075-81.

22. Halfvarson J, Bodin L, Tysk C, et al. Inflammatory bowel disease in a Swedish twin cohort: a long-term follow-up of concordance and clinical characteristics. Gastroenterology 2003;124:1767-73.

23. Monsen U, Brostrom O, Nordenvall B, et al. Prevalence of inflammatory bowel disease among relatives of patients with ulcerative colitis. Scand J Gastroenterol $1987 ; 22: 214-8$.

24. Tysk C, Lindberg E, Jarnerot G, et al. Ulcerative colitis and Crohn's disease in an unselected population of monozygotic and dizygotic twins. A study of heritability and the influence of smoking. Gut 1988;29:990-6.

25. Orholm M, Munkholm P, Langholz E, et al. Familial occurrence of inflammatory bowel disease. N Engl J Med 1991;324:84-8. 
26. Peeters M, Nevens H, Baert F, et al. Familial aggregation in Crohn's disease: increased age-adjusted risk and concordance in clinical characteristics. Gastroenterology 1996;111:597-603.

27. Franke A, McGovern DP, Barrett JC, et al. Genome-wide meta-analysis increases to 71 the number of confirmed Crohn's disease susceptibility loci. Nat Genet;42:1118-25.

28. Lee JC, Parkes M. Genome-wide association studies and Crohn's disease. Brief Funct Genomics;10:71-6.

29. Hugot JP, Chamaillard M, Zouali H, et al. Association of NOD2 leucine-rich repeat variants with susceptibility to Crohn's disease. Nature 2001;411:599-603.

30. Hugot JP, Laurent-Puig P, Gower-Rousseau C, et al. Mapping of a susceptibility locus for Crohn's disease on chromosome 16. Nature 1996;379:821-3.

31. Ogura Y, Bonen DK, Inohara N, et al. A frameshift mutation in NOD2 associated with susceptibility to Crohn's disease. Nature 2001;411:603-6.

32. Lala S, Ogura Y, Osborne C, et al. Crohn's disease and the NOD2 gene: a role for paneth cells. Gastroenterology 2003;125:47-57.

33. Inohara N, Ogura Y, Fontalba A, et al. Host recognition of bacterial muramyl dipeptide mediated through NOD2. Implications for Crohn's disease. J Biol Chem $2003 ; 278: 5509-12$.

34. Ogura Y, Inohara N, Benito A, et al. Nod2, a Nod1/Apaf-1 family member that is restricted to monocytes and activates NF-kappaB. J Biol Chem 2001;276:4812-8.

35. Zhang H MD, Tremelling M, Parkes M. Genetics of inflammatory bowel disease: clues to pathogenesis. British Medical Bulletin 2008;87:17-30. 
36. Leong RW, Armuzzi A, Ahmad T, et al. NOD2/CARD15 gene polymorphisms and Crohn's disease in the Chinese population. Aliment Pharmacol Ther 2003;17:1465-70.

37. Yamazaki K, Takazoe M, Tanaka T, et al. Absence of mutation in the NOD2/CARD15 gene among 483 Japanese patients with Crohn's disease. J Hum Genet 2002;47:469-72.

38. Hugot JP, Zaccaria I, Cavanaugh J, et al. Prevalence of CARD15/NOD2 mutations in Caucasian healthy people. Am J Gastroenterol 2007;102:1259-67.

39. Hedl M, Abraham C. Distinct roles for Nod2 protein and autocrine interleukin1beta in muramyl dipeptide-induced mitogen-activated protein kinase activation and cytokine secretion in human macrophages. J Biol Chem;286:26440-9.

40. Hedl M, Li J, Cho JH, et al. Chronic stimulation of Nod2 mediates tolerance to bacterial products. Proc Natl Acad Sci U S A 2007;104:19440-5.

41. Netea MG, Ferwerda G, de Jong DJ, et al. Nucleotide-binding oligomerization domain-2 modulates specific TLR pathways for the induction of cytokine release. J Immunol 2005;174:6518-23.

42. Pauleau AL, Murray PJ. Role of nod2 in the response of macrophages to toll-like receptor agonists. Mol Cell Biol 2003;23:7531-9.

43. Macho Fernandez E, Valenti V, Rockel C, et al. Anti-inflammatory capacity of selected lactobacilli in experimental colitis is driven by NOD2-mediated recognition of a specific peptidoglycan-derived muropeptide. Gut;60:1050-9.

44. Rehman A, Sina C, Gavrilova O, et al. Nod2 is essential for temporal development of intestinal microbial communities. Gut;60:1354-62. 
45. Kobayashi KS, Chamaillard M, Ogura Y, et al. Nod2-dependent regulation of innate and adaptive immunity in the intestinal tract. Science 2005;307:731-4.

46. Petnicki-Ocwieja T, Hrncir T, Liu YJ, et al. Nod2 is required for the regulation of commensal microbiota in the intestine. Proc Natl Acad Sci U S A 2009; 106:15813-8.

47. Barreau F, Madre C, Meinzer U, et al. Nod2 regulates the host response towards microflora by modulating $\mathrm{T}$ cell function and epithelial permeability in mouse Peyer's patches. Gut;59:207-17.

48. Hampe J, Franke A, Rosenstiel P, et al. A genome-wide association scan of nonsynonymous SNPs identifies a susceptibility variant for Crohn disease in ATG16L1. Nat Genet 2007;39:207-11.

49. Rioux JD, Xavier RJ, Taylor KD, et al. Genome-wide association study identifies new susceptibility loci for Crohn disease and implicates autophagy in disease pathogenesis. Nat Genet 2007;39:596-604.

50. Genome-wide association study of 14,000 cases of seven common diseases and 3,000 shared controls. Nature 2007;447:661-78.

51. Gutierrez MG, Master SS, Singh SB, et al. Autophagy is a defense mechanism inhibiting BCG and Mycobacterium tuberculosis survival in infected macrophages. Cell 2004;119:753-66.

52. Singh SB, Davis AS, Taylor GA, et al. Human IRGM induces autophagy to eliminate intracellular mycobacteria. Science 2006;313:1438-41. 
53. McCarroll SA, Huett A, Kuballa P, et al. Deletion polymorphism upstream of IRGM associated with altered IRGM expression and Crohn's disease. Nat Genet 2008;40:1107-12.

54. Travassos LH, Carneiro LA, Ramjeet M, et al. Nod1 and Nod2 direct autophagy by recruiting ATG16L1 to the plasma membrane at the site of bacterial entry. Nat Immunol;11:55-62.

55. Cooney R, Baker J, Brain O, et al. NOD2 stimulation induces autophagy in dendritic cells influencing bacterial handling and antigen presentation. Nat Med;16:90-7.

56. Hendrickson BA, Gokhale R, Cho JH. Clinical aspects and pathophysiology of inflammatory bowel disease. Clin Microbiol Rev 2002;15:79-94.

57. Hubbard J, Surawicz CM. Etiological role of mycobacterium in Crohn's disease: An assessment of the literature. Dig Dis 1999;17:6-13.

58. Van Kruiningen HJ. Lack of support for a common etiology in Johne's disease of animals and Crohn's disease in humans. Inflamm Bowel Dis 1999;5:183-91.

59. Montgomery SM, Morris DL, Pounder RE, et al. Paramyxovirus infections in childhood and subsequent inflammatory bowel disease. Gastroenterology 1999;116:796-803.

60. Thompson NP, Montgomery SM, Pounder RE, et al. Is measles vaccination a risk factor for inflammatory bowel disease? Lancet 1995;345:1071-4.

61. Yanai H, Shimizu N, Nagasaki S, et al. Epstein-Barr virus infection of the colon with inflammatory bowel disease. Am J Gastroenterol 1999;94:1582-6. 
62. Lawlor G, Moss AC. Cytomegalovirus in inflammatory bowel disease: pathogen or innocent bystander? Inflamm Bowel Dis;16:1620-7.

63. Afzal MA, Armitage E, Begley J, et al. Absence of detectable measles virus genome sequence in inflammatory bowel disease tissues and peripheral blood lymphocytes. J Med Virol 1998;55:243-9.

64. Afzal MA, Armitage E, Ghosh S, et al. Further evidence of the absence of measles virus genome sequence in full thickness intestinal specimens from patients with Crohn's disease. J Med Virol 2000;62:377-82.

65. Afzal MA, Minor PD, Begley J, et al. Absence of measles-virus genome in inflammatory bowel disease. Lancet 1998;351:646-7.

66. Folwaczny C, Loeschke K, Schnettler D, et al. Endothelial cell autoantibodies are a marker of disease susceptibility in inflammatory bowel disease but apparently not linked to persistent measles virus infection. Clin Immunol 2000;95:197-202.

67. Folwaczny C, Noehl N, Tschop K, et al. Goblet cell autoantibodies in patients with inflammatory bowel disease and their first-degree relatives.

Gastroenterology 1997;113:101-6.

68. Khoo UY, Bjarnason I, Donaghy A, et al. Antibodies to colonic epithelial cells from the serum and colonic mucosal washings in ulcerative colitis. Gut 1995;37:63-70.

69. Onuma EK, Amenta PS, Ramaswamy K, et al. Autoimmunity in ulcerative colitis (UC): a predominant colonic mucosal B cell response against human tropomyosin isoform 5. Clin Exp Immunol 2000;121:466-71. 
70. Olives JP, Breton A, Hugot JP, et al. Antineutrophil cytoplasmic antibodies in children with inflammatory bowel disease: prevalence and diagnostic value. $\mathbf{J}$ Pediatr Gastroenterol Nutr 1997;25:142-8.

71. MacDonald TT, Monteleone G, Pender SL. Recent developments in the immunology of inflammatory bowel disease. Scand J Immunol 2000;51:2-9.

72. Roozendaal C, Pogany K, Hummel EJ, et al. Titres of anti-neutrophil cytoplasmic antibodies in inflammatory bowel disease are not related to disease activity. QJM 1999;92:651-8.

73. Schultsz C, Van Den Berg FM, Ten Kate FW, et al. The intestinal mucus layer from patients with inflammatory bowel disease harbors high numbers of bacteria compared with controls. Gastroenterology 1999;117:1089-97.

74. Schultsz C, Moussa M, van Ketel R, et al. Frequency of pathogenic and enteroadherent Escherichia coli in patients with inflammatory bowel disease and controls. J Clin Pathol 1997;50:573-9.

75. Cartun RW, Van Kruiningen HJ, Pedersen CA, et al. An immunocytochemical search for infectious agents in Crohn's disease. Mod Pathol 1993;6:212-9.

76. Liu Y, van Kruiningen HJ, West AB, et al. Immunocytochemical evidence of Listeria, Escherichia coli, and Streptococcus antigens in Crohn's disease. Gastroenterology 1995;108:1396-404.

77. Sartor RB. Review article: Role of the enteric microflora in the pathogenesis of intestinal inflammation and arthritis. Aliment Pharmacol Ther 1997;11 Suppl 3:17-22; discussion 22-3. 
78. Thayer WR, Jr., Chitnavis V. Inflammatory bowel disease. The case for an infectious etiology. Med Clin North Am 1994;78:1233-47.

79. Jergens AE, Dorn A, Wilson J, et al. Induction of differential immune reactivity to members of the flora of gnotobiotic mice following colonization with Helicobacter bilis or Brachyspira hyodysenteriae. Microbes Infect 2006;8:160210.

80. Jergens AE, Wilson-Welder JH, Dorn A, et al. Helicobacter bilis triggers persistent immune reactivity to antigens derived from the commensal bacteria in gnotobiotic C3H/HeN mice. Gut 2007;56:934-40.

81. Rocha F, Laughlin R, Musch MW, et al. Surgical stress shifts the intestinal Escherichia coli population to that of a more adherent phenotype: role in barrier regulation. Surgery 2001;130:65-73.

82. Ohkusa T, Fujimoto H, Shikato F, et al. [Production of erosions and ulcers of the colon in Syrian hamsters with Bacteroides vulgatus isolated from the fecal microflora of human ulcerative colitis]. Nihon Shokakibyo Gakkai Zasshi 1985;82:72-7.

83. Okayasu I, Hatakeyama S, Yamada M, et al. A novel method in the induction of reliable experimental acute and chronic ulcerative colitis in mice. Gastroenterology 1990;98:694-702.

84. Mahler M, Bristol IJ, Leiter EH, et al. Differential susceptibility of inbred mouse strains to dextran sulfate sodium-induced colitis. Am J Physiol 1998;274:G54451. 
85. Kitajima S, Takuma S, Morimoto M. Histological analysis of murine colitis induced by dextran sulfate sodium of different molecular weights. Exp Anim 2000;49:9-15.

86. Vowinkel T, Kalogeris TJ, Mori M, et al. Impact of dextran sulfate sodium load on the severity of inflammation in experimental colitis. Dig Dis Sci 2004;49:55664.

87. Melgar S, Engstrom K, Jagervall A, et al. Psychological stress reactivates dextran sulfate sodium-induced chronic colitis in mice. Stress 2008;11:348-62.

88. Kitajima S, Takuma S, Morimoto M. Tissue distribution of dextran sulfate sodium (DSS) in the acute phase of murine DSS-induced colitis. J Vet Med Sci 1999;61:67-70.

89. Cooper HS, Murthy SN, Shah RS, et al. Clinicopathologic study of dextran sulfate sodium experimental murine colitis. Lab Invest 1993;69:238-49.

90. Hans W, Scholmerich J, Gross V, et al. The role of the resident intestinal flora in acute and chronic dextran sulfate sodium-induced colitis in mice. Eur J Gastroenterol Hepatol 2000;12:267-73.

91. Scheiffele F, Fuss IJ. Induction of TNBS colitis in mice. Curr Protoc Immunol 2002; Chapter 15:Unit 1519.

92. Neurath MF, Fuss I, Kelsall BL, et al. Experimental granulomatous colitis in mice is abrogated by induction of TGF-beta-mediated oral tolerance. $\mathrm{J}$ Exp Med $1996 ; 183: 2605-16$. 
93. Elson CO, Beagley KW, Sharmanov AT, et al. Hapten-induced model of murine inflammatory bowel disease: mucosa immune responses and protection by tolerance. J Immunol 1996;157:2174-85.

94. Parronchi P, Romagnani P, Annunziato F, et al. Type $1 \mathrm{~T}$-helper cell predominance and interleukin-12 expression in the gut of patients with Crohn's disease. Am J Pathol 1997;150:823-32.

95. Fuss IJ, Neurath M, Boirivant M, et al. Disparate CD4+ lamina propria (LP) lymphokine secretion profiles in inflammatory bowel disease. Crohn's disease LP cells manifest increased secretion of IFN-gamma, whereas ulcerative colitis LP cells manifest increased secretion of IL-5. J Immunol 1996;157:1261-70.

96. Ehrhardt RO, Ludviksson BR, Gray B, et al. Induction and prevention of colonic inflammation in IL-2-deficient mice. J Immunol 1997;158:566-73.

97. Hornquist CE, Lu X, Rogers-Fani PM, et al. G(alpha)i2-deficient mice with colitis exhibit a local increase in memory CD4+ T cells and proinflammatory Th1-type cytokines. J Immunol 1997;158:1068-77.

98. Mizoguchi A, Mizoguchi E, Chiba C, et al. Cytokine imbalance and autoantibody production in $\mathrm{T}$ cell receptor-alpha mutant mice with inflammatory bowel disease. J Exp Med 1996;183:847-56.

99. Mombaerts P, Mizoguchi E, Grusby MJ, et al. Spontaneous development of inflammatory bowel disease in T cell receptor mutant mice. Cell 1993;75:274-82.

100. Panwala CM, Jones JC, Viney JL. A novel model of inflammatory bowel disease: mice deficient for the multiple drug resistance gene, mdr1a, spontaneously develop colitis. J Immunol 1998;161:5733-44. 
101. Van der Sluis M, De Koning BA, De Bruijn AC, et al. Muc2-deficient mice spontaneously develop colitis, indicating that MUC2 is critical for colonic protection. Gastroenterology 2006;131:117-29.

102. Mashimo H, Wu DC, Podolsky DK, et al. Impaired defense of intestinal mucosa in mice lacking intestinal trefoil factor. Science 1996;274:262-5.

103. Kontoyiannis D, Pasparakis M, Pizarro TT, et al. Impaired on/off regulation of TNF biosynthesis in mice lacking TNF AU-rich elements: implications for joint and gut-associated immunopathologies. Immunity 1999;10:387-98.

104. Wirtz S, Finotto S, Kanzler S, et al. Cutting edge: chronic intestinal inflammation in STAT-4 transgenic mice: characterization of disease and adoptive transfer by TNF- plus IFN-gamma-producing CD4+ T cells that respond to bacterial antigens. J Immunol 1999;162:1884-8.

105. Kuhn R, Lohler J, Rennick D, et al. Interleukin-10-deficient mice develop chronic enterocolitis. Cell 1993;75:263-74.

106. Spencer SD, Di Marco F, Hooley J, et al. The orphan receptor CRF2-4 is an essential subunit of the interleukin 10 receptor. J Exp Med 1998;187:571-8.

107. Rennick DM, Fort MM. Lessons from genetically engineered animal models. XII. IL-10-deficient (IL-10(-/-) mice and intestinal inflammation. Am J Physiol Gastrointest Liver Physiol 2000;278:G829-33.

108. Kullberg MC, Ward JM, Gorelick PL, et al. Helicobacter hepaticus triggers colitis in specific-pathogen-free interleukin-10 (IL-10)-deficient mice through an IL-12and gamma interferon-dependent mechanism. Infect Immun 1998;66:5157-66. 
109. Davidson NJ, Leach MW, Fort MM, et al. T helper cell 1-type CD4+ T cells, but not B cells, mediate colitis in interleukin 10-deficient mice. J Exp Med 1996;184:241-51.

110. Kullberg MC, Rothfuchs AG, Jankovic D, et al. Helicobacter hepaticus-induced colitis in interleukin-10-deficient mice: cytokine requirements for the induction and maintenance of intestinal inflammation. Infect Immun 2001;69:4232-41.

111. Kullberg MC, Jankovic D, Feng CG, et al. IL-23 plays a key role in Helicobacter hepaticus-induced T cell-dependent colitis. J Exp Med 2006;203:2485-94.

112. Mills KH. Induction, function and regulation of IL-17-producing T cells. Eur J Immunol 2008;38:2636-49.

113. Fiorentino DF, Zlotnik A, Vieira P, et al. IL-10 acts on the antigen-presenting cell to inhibit cytokine production by Th1 cells. J Immunol 1991;146:3444-51.

114. Sellon RK, Tonkonogy S, Schultz M, et al. Resident enteric bacteria are necessary for development of spontaneous colitis and immune system activation in interleukin-10-deficient mice. Infect Immun 1998;66:5224-31.

115. Schultz M, Tonkonogy SL, Sellon RK, et al. IL-2-deficient mice raised under germfree conditions develop delayed mild focal intestinal inflammation. Am J Physiol 1999;276:G1461-72.

116. Dianda L, Hanby AM, Wright NA, et al. T cell receptor-alpha beta-deficient mice fail to develop colitis in the absence of a microbial environment. Am J Pathol 1997;150:91-7. 
117. Aranda R, Sydora BC, McAllister PL, et al. Analysis of intestinal lymphocytes in mouse colitis mediated by transfer of CD4+, CD45RBhigh T cells to SCID recipients. J Immunol 1997;158:3464-73.

118. Bamias G, Okazawa A, Rivera-Nieves J, et al. Commensal bacteria exacerbate intestinal inflammation but are not essential for the development of murine ileitis. J Immunol 2007;178:1809-18.

119. Erdman S, Fox JG, Dangler CA, et al. Typhlocolitis in NF-kappa B-deficient mice. J Immunol 2001;166:1443-7.

120. Fox JG, Gorelick PL, Kullberg MC, et al. A novel urease-negative Helicobacter species associated with colitis and typhlitis in IL-10-deficient mice. Infect Immun 1999;67:1757-62.

121. Burich A, Hershberg R, Waggie K, et al. Helicobacter-induced inflammatory bowel disease in IL-10- and T cell-deficient mice. Am J Physiol Gastrointest Liver Physiol 2001;281:G764-78.

122. Chin EY, Dangler CA, Fox JG, et al. Helicobacter hepaticus infection triggers inflammatory bowel disease in $\mathrm{T}$ cell receptor alphabeta mutant mice. Comp Med 2000;50:586-94.

123. Dieleman LA, Arends A, Tonkonogy SL, et al. Helicobacter hepaticus does not induce or potentiate colitis in interleukin-10-deficient mice. Infect Immun 2000;68:5107-13.

124. Iwasaki A, Kelsall BL. Unique functions of CD11b+, CD8 alpha+, and doublenegative Peyer's patch dendritic cells. J Immunol 2001;166:4884-90. 
125. Karlis J, Penttila I, Tran TB, et al. Characterization of colonic and mesenteric lymph node dendritic cell subpopulations in a murine adoptive transfer model of inflammatory bowel disease. Inflamm Bowel Dis 2004;10:834-47.

126. Iwasaki A, Kelsall BL. Localization of distinct Peyer's patch dendritic cell subsets and their recruitment by chemokines macrophage inflammatory protein (MIP)3alpha, MIP-3beta, and secondary lymphoid organ chemokine. J Exp Med 2000;191:1381-94.

127. Sato A, Hashiguchi M, Toda E, et al. CD11b+ Peyer's patch dendritic cells secrete IL-6 and induce IgA secretion from naive B cells. J Immunol 2003;171:3684-90.

128. Sato A, Iwasaki A. Peyer's patch dendritic cells as regulators of mucosal adaptive immunity. Cell Mol Life Sci 2005;62:1333-8.

129. Zhao X, Sato A, Dela Cruz CS, et al. CCL9 is secreted by the follicle-associated epithelium and recruits dome region Peyer's patch CD11b+ dendritic cells. J Immunol 2003;171:2797-803.

130. Becker C, Wirtz S, Blessing M, et al. Constitutive p40 promoter activation and IL-23 production in the terminal ileum mediated by dendritic cells. J Clin Invest 2003;112:693-706.

131. Myles MH, Dieckgraefe BK, Criley JM, et al. Characterization of cecal gene expression in a differentially susceptible mouse model of bacterial-induced inflammatory bowel disease. Inflamm Bowel Dis 2007;13:822-36.

132. Livingston RS, Myles MH, Livingston BA, et al. Sex influence on chronic intestinal inflammation in Helicobacter hepaticus-infected $\mathrm{A} / \mathrm{JCr}$ mice. Comp Med 2004;54:301-8. 
133. Loftus EV, Jr. Clinical epidemiology of inflammatory bowel disease: Incidence, prevalence, and environmental influences. Gastroenterology 2004;126:1504-17.

134. Germano G, Allavena P, Mantovani A. Cytokines as a key component of cancerrelated inflammation. Cytokine 2008;43:374-9.

135. Karin M, Greten FR. NF-kappaB: linking inflammation and immunity to cancer development and progression. Nat Rev Immunol 2005;5:749-59.

136. Mantovani A, Allavena P, Sica A, et al. Cancer-related inflammation. Nature $2008 ; 454: 436-44$.

137. Hartnett L, Egan LJ. Inflammation, DNA methylation and colitis-associated cancer. Carcinogenesis;33:723-31.

138. Zhang HY, Spechler SJ, Souza RF. Esophageal adenocarcinoma arising in Barrett esophagus. Cancer Lett 2009;275:170-7.

139. Genta RM. The gastritis connection: prevention and early detection of gastric neoplasms. J Clin Gastroenterol 2003;36:S44-9; discussion S61-2.

140. Bernstein CN, Blanchard JF, Kliewer E, et al. Cancer risk in patients with inflammatory bowel disease: a population-based study. Cancer 2001;91:854-62.

141. Itzkowitz SH. Molecular biology of dysplasia and cancer in inflammatory bowel disease. Gastroenterol Clin North Am 2006;35:553-71.

142. Itzkowitz SH, Yio X. Inflammation and cancer IV. Colorectal cancer in inflammatory bowel disease: the role of inflammation. Am J Physiol Gastrointest Liver Physiol 2004;287:G7-17. 
143. Aust DE, Terdiman JP, Willenbucher RF, et al. The APC/beta-catenin pathway in ulcerative colitis-related colorectal carcinomas: a mutational analysis. Cancer 2002;94:1421-7.

144. Tarmin L, Yin J, Harpaz N, et al. Adenomatous polyposis coli gene mutations in ulcerative colitis-associated dysplasias and cancers versus sporadic colon neoplasms. Cancer Res 1995;55:2035-8.

145. Brentnall TA, Crispin DA, Rabinovitch PS, et al. Mutations in the p53 gene: an early marker of neoplastic progression in ulcerative colitis. Gastroenterology 1994;107:369-78.

146. Nuako KW, Ahlquist DA, Mahoney DW, et al. Familial predisposition for colorectal cancer in chronic ulcerative colitis: a case-control study. Gastroenterology 1998;115:1079-83.

147. Genoa A, Seth, K., Schmidt, U., Carles, M., Gwathmey, J.K. Dilated cardiomyopathy in turkeys: an animal model forthe study of human heart failure. Laboratory Animal Science 1996;46:399-404.

148. Okamoto K, Anki, K. Development of a strain of spontaneously hypertensive rats. Jpn Circ. J 1963;27:282-293.

149. Crespo MJ, Cruz N, Altieri PI, et al. Chronic treatment with N-acetylcysteine improves cardiac function but does not prevent progression of cardiomyopathy in Syrian cardiomyopathic hamsters. J Cardiovasc Pharmacol Ther;16:197-204.

150. Geurts AM, Cost GJ, Freyvert Y, et al. Knockout rats via embryo microinjection of zinc-finger nucleases. Science 2009;325:433. 
151. Orban PC, Chui D, Marth JD. Tissue- and site-specific DNA recombination in transgenic mice. Proc Natl Acad Sci U S A 1992;89:6861-5.

152. Legault F, Rouleau JL, Juneau C, et al. Functional and morphological characteristics of compensated and decompensated cardiac hypertrophy in dogs with chronic infrarenal aorto-caval fistulas. Circ Res 1990;66:846-59.

153. Bajusz E. Enhanced healing of myocardial lesions and prevention of congestive heart failure. Experimental studies on the therapeutic effect of K-salts, alone and in combination with glucose and insulin, in a hereditary cardiomyopathy. Arzneimittelforschung 1969;19:1830-6.

154. Bajusz E. Hereditary cardiomyopathy: a new disease model. Am Heart J 1969;77:686-96.

155. Zhu Y, Richardson JA, Parada LF, et al. Smad3 mutant mice develop metastatic colorectal cancer. Cell 1998;94:703-14.

156. Maggio-Price L, Treuting P, Zeng W, et al. Helicobacter infection is required for inflammation and colon cancer in SMAD3-deficient mice. Cancer Res 2006;66:828-38.

157. Farraye FA, Odze RD, Eaden J, et al. AGA medical position statement on the diagnosis and management of colorectal neoplasia in inflammatory bowel disease. Gastroenterology;138:738-45.

158. Reuter BK, Pizarro TT. Commentary: the role of the IL-18 system and other members of the IL-1R/TLR superfamily in innate mucosal immunity and the pathogenesis of inflammatory bowel disease: friend or foe? Eur J Immunol 2004;34:2347-55. 
159. Gillen CD, Andrews HA, Prior P, et al. Crohn's disease and colorectal cancer. Gut 1994;35:651-5.

160. Kotlowski R, Bernstein CN, Sepehri S, et al. High prevalence of Escherichia coli belonging to the $\mathrm{B} 2+\mathrm{D}$ phylogenetic group in inflammatory bowel disease. Gut 2007;56:669-75.

161. Sepehri S, Kotlowski R, Bernstein CN, et al. Phylogenetic analysis of inflammatory bowel disease associated Escherichia coli and the fimH virulence determinant. Inflamm Bowel Dis 2009.

162. La Ferla K, Seegert D, Schreiber S. Activation of NF-kappaB in intestinal epithelial cells by E. coli strains isolated from the colonic mucosa of IBD patients. Int J Colorectal Dis 2004;19:334-42.

163. Chiodini RJ. Crohn's disease and the mycobacterioses: a review and comparison of two disease entities. Clin Microbiol Rev 1989;2:90-117.

164. Chiodini RJ, Van Kruiningen HJ, Thayer WR, et al. Spheroplastic phase of mycobacteria isolated from patients with Crohn's disease. J Clin Microbiol 1986;24:357-63.

165. Rowbotham DS, Mapstone NP, Trejdosiewicz LK, et al. Mycobacterium paratuberculosis DNA not detected in Crohn's disease tissue by fluorescent polymerase chain reaction. Gut 1995;37:660-7.

166. Stainsby KJ, Lowes JR, Allan RN, et al. Antibodies to Mycobacterium paratuberculosis and nine species of environmental mycobacteria in Crohn's disease and control subjects. Gut 1993;34:371-4. 
167. Halme L, Rautelin H, Leidenius M, et al. Inverse correlation between Helicobacter pylori infection and inflammatory bowel disease. J Clin Pathol 1996;49:65-7.

168. Duchmann R, Marker-Hermann E, Meyer zum Buschenfelde KH. Bacteriaspecific T-cell clones are selective in their reactivity towards different enterobacteria or $\mathrm{H}$. pylori and increased in inflammatory bowel disease. Scand $\mathbf{J}$ Immunol 1996;44:71-9.

169. Pearce CB, Duncan HD, Timmis L, et al. Assessment of the prevalence of infection with Helicobacter pylori in patients with inflammatory bowel disease. Eur J Gastroenterol Hepatol 2000;12:439-43.

170. Helio T, Halme L, Lappalainen M, et al. CARD15/NOD2 gene variants are associated with familially occurring and complicated forms of Crohn's disease. Gut 2003;52:558-62.

171. Papaconstantinou I, Theodoropoulos G, Gazouli M, et al. Association between mutations in the CARD15/NOD2 gene and colorectal cancer in a Greek population. Int J Cancer 2005;114:433-5.

172. Gewirtz AT, Vijay-Kumar M, Brant SR, et al. Dominant-negative TLR5 polymorphism reduces adaptive immune response to flagellin and negatively associates with Crohn's disease. Am J Physiol Gastrointest Liver Physiol 2006;290:G1157-63.

173. Franchimont D, Vermeire S, El Housni H, et al. Deficient host-bacteria interactions in inflammatory bowel disease? The toll-like receptor (TLR)-4 
Asp299gly polymorphism is associated with Crohn's disease and ulcerative colitis. Gut 2004;53:987-92.

174. De Jager PL, Franchimont D, Waliszewska A, et al. The role of the Toll receptor pathway in susceptibility to inflammatory bowel diseases. Genes Immun 2007;8:387-97.

175. Torok HP, Glas J, Tonenchi L, et al. Polymorphisms of the lipopolysaccharidesignaling complex in inflammatory bowel disease: association of a mutation in the Toll-like receptor 4 gene with ulcerative colitis. Clin Immunol 2004;112:85-91.

176. Gazouli M, Mantzaris G, Kotsinas A, et al. Association between polymorphisms in the Toll-like receptor 4, CD14, and CARD15/NOD2 and inflammatory bowel disease in the Greek population. World J Gastroenterol 2005;11:681-5.

177. Duerr RH, Taylor KD, Brant SR, et al. A genome-wide association study identifies IL23R as an inflammatory bowel disease gene. Science 2006;314:14613.

178. Oliver J, Rueda B, Lopez-Nevot MA, et al. Replication of an association between IL23R gene polymorphism with inflammatory bowel disease. Clin Gastroenterol Hepatol 2007;5:977-81, 981 e1-2.

179. Marquez A, Mendoza JL, Taxonera C, et al. IL23R and IL12B polymorphisms in Spanish IBD patients: no evidence of interaction. Inflamm Bowel Dis 2008;14:1192-6.

180. Cummings JR, Ahmad T, Geremia A, et al. Contribution of the novel inflammatory bowel disease gene IL23R to disease susceptibility and phenotype. Inflamm Bowel Dis 2007;13:1063-8. 
181. Roberts RL, Gearry RB, Hollis-Moffatt JE, et al. IL23R R381Q and ATG16L1 T300A are strongly associated with Crohn's disease in a study of New Zealand Caucasians with inflammatory bowel disease. Am J Gastroenterol 2007; 102:2754-61.

182. Myles MH, Livingston RS, Livingston BA, et al. Analysis of gene expression in ceca of Helicobacter hepaticus-infected $\mathrm{A} / \mathrm{JCr}$ mice before and after development of typhlitis. Infect Immun 2003;71:3885-93.

183. Chung Y, Chang JH, Kweon MN, et al. CD8alpha-11b+ dendritic cells but not CD8alpha+ dendritic cells mediate cross-tolerance toward intestinal antigens. Blood 2005;106:201-6.

184. Ehirchiou D, Xiong Y, Xu G, et al. CD11b facilitates the development of peripheral tolerance by suppressing Th17 differentiation. J Exp Med 2007;204:1519-24.

185. Fleeton MN, Contractor N, Leon F, et al. Peyer's patch dendritic cells process viral antigen from apoptotic epithelial cells in the intestine of reovirus-infected mice. J Exp Med 2004;200:235-45.

186. Livingston RS, Riley LK, Steffen EK, et al. Serodiagnosis of Helicobacter hepaticus infection in mice by an enzyme-linked immunosorbent assay. J Clin Microbiol 1997;35:1236-8.

187. Riley LK, Franklin CL, Hook RR, Jr., et al. Identification of murine helicobacters by PCR and restriction enzyme analyses. J Clin Microbiol 1996;34:942-6. 
188. Jung S, Unutmaz D, Wong P, et al. In vivo depletion of CD11c(+) dendritic cells abrogates priming of CD8(+) T cells by exogenous cell-associated antigens. Immunity 2002;17:211-20.

189. Hildner K, Edelson BT, Purtha WE, et al. Batf3 deficiency reveals a critical role for CD8alpha+ dendritic cells in cytotoxic T cell immunity. Science 2008;322:1097-100.

190. Shortman K, Naik SH. Steady-state and inflammatory dendritic-cell development. Nat Rev Immunol 2007;7:19-30.

191. Pulendran B. Variegation of the immune response with dendritic cells and pathogen recognition receptors. J Immunol 2005;174:2457-65.

192. Pulendran B, Lingappa J, Kennedy MK, et al. Developmental pathways of dendritic cells in vivo: distinct function, phenotype, and localization of dendritic cell subsets in FLT3 ligand-treated mice. J Immunol 1997;159:2222-31.

193. Pulendran B, Smith JL, Caspary G, et al. Distinct dendritic cell subsets differentially regulate the class of immune response in vivo. Proc Natl Acad Sci U S A 1999;96:1036-41.

194. Maldonado-Lopez R, De Smedt T, Michel P, et al. CD8alpha+ and CD8alphasubclasses of dendritic cells direct the development of distinct $\mathrm{T}$ helper cells in vivo. J Exp Med 1999;189:587-92.

195. Maldonado-Lopez R, De Smedt T, Pajak B, et al. Role of CD8alpha+ and CD8alpha- dendritic cells in the induction of primary immune responses in vivo. $\mathrm{J}$ Leukoc Biol 1999;66:242-6. 
196. Maldonado-Lopez R, Moser M. Dendritic cell subsets and the regulation of Th1/Th2 responses. Semin Immunol 2001;13:275-82.

197. Pulendran B, Tang H, Denning TL. Division of labor, plasticity, and crosstalk between dendritic cell subsets. Curr Opin Immunol 2008;20:61-7.

198. De Smedt T, Butz E, Smith J, et al. CD8alpha(-) and CD8alpha(+) subclasses of dendritic cells undergo phenotypic and functional maturation in vitro and in vivo. J Leukoc Biol 2001;69:951-8.

199. Iliev ID, Spadoni I, Mileti E, et al. Human intestinal epithelial cells promote the differentiation of tolerogenic dendritic cells. Gut 2009.

200. Rescigno M. Intestinal epithelial cells control dendritic cell function. J Pediatr Gastroenterol Nutr 2008;46 Suppl 1:E17-9.

201. Rimoldi M, Chieppa M, Salucci V, et al. Intestinal immune homeostasis is regulated by the crosstalk between epithelial cells and dendritic cells. Nat Immunol 2005;6:507-14.

202. Rimoldi M, Chieppa M, Vulcano M, et al. Intestinal epithelial cells control dendritic cell function. Ann N Y Acad Sci 2004;1029:66-74.

203. Monteleone I, Vavassori P, Biancone L, et al. Immunoregulation in the gut: success and failures in human disease. Gut 2002;50 Suppl 3:III60-4.

204. Iwasaki A. Mucosal dendritic cells. Annu Rev Immunol 2007;25:381-418.

205. Kawashima D, Oshitani N, Jinno Y, et al. Augmented expression of secondary lymphoid tissue chemokine and EBI1 ligand chemokine in Crohn's disease. J Clin Pathol 2005;58:1057-63. 
206. Middel P, Raddatz D, Gunawan B, et al. Increased number of mature dendritic cells in Crohn's disease: evidence for a chemokine mediated retention mechanism. Gut 2006;55:220-7.

207. Kwon JH, Keates S, Bassani L, et al. Colonic epithelial cells are a major site of macrophage inflammatory protein 3alpha (MIP-3alpha) production in normal colon and inflammatory bowel disease. Gut 2002;51:818-26.

208. Jang MH, Sougawa N, Tanaka T, et al. CCR7 is critically important for migration of dendritic cells in intestinal lamina propria to mesenteric lymph nodes. $\mathbf{J}$ Immunol 2006;176:803-10.

209. Macpherson AJ, Uhr T. Induction of protective IgA by intestinal dendritic cells carrying commensal bacteria. Science 2004;303:1662-5.

210. Guarner F, Malagelada JR. Gut flora in health and disease. Lancet 2003;361:5129.

211. Annacker O, Coombes JL, Malmstrom V, et al. Essential role for CD103 in the T cell-mediated regulation of experimental colitis. J Exp Med 2005;202:1051-61.

212. Drakes ML, Blanchard TG, Czinn SJ. Colon lamina propria dendritic cells induce a proinflammatory cytokine response in lamina propria T cells in the SCID mouse model of colitis. J Leukoc Biol 2005;78:1291-300.

213. Denning TL, Wang YC, Patel SR, et al. Lamina propria macrophages and dendritic cells differentially induce regulatory and interleukin 17-producing T cell responses. Nat Immunol 2007;8:1086-94. 
214. Kim U, Wang Y, Sanford T, et al. Molecular cloning of cDNA for doublestranded RNA adenosine deaminase, a candidate enzyme for nuclear RNA editing. Proc Natl Acad Sci U S A 1994;91:11457-61.

215. Slezak-Prochazka I, Durmus S, Kroesen BJ, et al. MicroRNAs, macrocontrol: regulation of miRNA processing. RNA 2010;16:1087-95.

216. Komatsu M, Kobayashi D, Saito K, et al. Tumor necrosis factor-alpha in serum of patients with inflammatory bowel disease as measured by a highly sensitive immuno-PCR. Clin Chem 2001;47:1297-301.

217. Braegger CP, Nicholls S, Murch SH, et al. Tumour necrosis factor alpha in stool as a marker of intestinal inflammation. Lancet 1992;339:89-91.

218. van Heel DA, Udalova IA, De Silva AP, et al. Inflammatory bowel disease is associated with a TNF polymorphism that affects an interaction between the OCT1 and NF(-kappa)B transcription factors. Hum Mol Genet 2002;11:1281-9.

219. Faunce DE, Stein-Streilein J. NKT cell-derived RANTES recruits APCs and $\mathrm{CD} 8+\mathrm{T}$ cells to the spleen during the generation of regulatory $\mathrm{T}$ cells in tolerance. J Immunol 2002;169:31-8.

220. Roda JM, Parihar R, Magro C, et al. Natural killer cells produce T cell-recruiting chemokines in response to antibody-coated tumor cells. Cancer Res 2006;66:51726.

221. Van Kaer L. Natural killer $\mathrm{T}$ cells as targets for immunotherapy of autoimmune diseases. Immunol Cell Biol 2004;82:315-22. 
222. Takayama T, Kamada N, Chinen H, et al. Imbalance of NKp44(+)NKp46(-) and NKp44(-)NKp46(+) natural killer cells in the intestinal mucosa of patients with Crohn's disease. Gastroenterology 2010;139:882-92, 892 e1-3.

223. Larmonier CB, Laubitz D, Thurston RD, et al. NHE3 modulates the severity of colitis in IL-10-deficient mice. Am J Physiol Gastrointest Liver Physiol 2011;300:G998-G1009.

224. Lee JW, Wang P, Kattah MG, et al. Differential regulation of chemokines by IL17 in colonic epithelial cells. J Immunol 2008;181:6536-45.

225. Tilg H, Trehu E, Atkins MB, et al. Interleukin-6 (IL-6) as an anti-inflammatory cytokine: induction of circulating IL-1 receptor antagonist and soluble tumor necrosis factor receptor p55. Blood 1994;83:113-8.

226. Toh ML, Kawashima M, Zrioual S, et al. IL-17 inhibits human Th1 differentiation through IL-12R beta 2 downregulation. Cytokine 2009;48:226-30.

227. Walsh JM, Terdiman JP. Colorectal cancer screening: scientific review. JAMA 2003;289:1288-96.

228. Dukes CE. The classification of cancer of the rectum. Journal of Pathology and Bacteriology 1932;35:323.

229. Astler VB, Coller FA. The prognostic significance of direct extension of carcinoma of the colon and rectum. Ann Surg 1954;139:846-52.

230. Ouyang DL, Chen JJ, Getzenberg RH, et al. Noninvasive testing for colorectal cancer: a review. Am J Gastroenterol 2005;100:1393-403. 
231. Burch JA, Soares-Weiser K, St John DJ, et al. Diagnostic accuracy of faecal occult blood tests used in screening for colorectal cancer: a systematic review. J Med Screen 2007;14:132-7.

232. Doria-Rose VP, Newcomb PA, Levin TR. Incomplete screening flexible sigmoidoscopy associated with female sex, age, and increased risk of colorectal cancer. Gut 2005;54:1273-8.

233. Huang EH, Carter JJ, Whelan RL, et al. Colonoscopy in mice. Surg Endosc 2002;16:22-4.

234. Becker C, Fantini MC, Neurath MF. High resolution colonoscopy in live mice. Nat Protoc 2006;1:2900-4.

235. Datto MB, Frederick JP, Pan L, et al. Targeted disruption of Smad3 reveals an essential role in transforming growth factor beta-mediated signal transduction. Mol Cell Biol 1999;19:2495-504.

236. Fox JG, Yan L, Shames B, et al. Persistent hepatitis and enterocolitis in germfree mice infected with Helicobacter hepaticus. Infect Immun 1996;64:3673-81.

237. Grady WM, Rajput A, Myeroff L, et al. Mutation of the type II transforming growth factor-beta receptor is coincident with the transformation of human colon adenomas to malignant carcinomas. Cancer Res 1998;58:3101-4.

238. Markowitz SD, Bertagnolli MM. Molecular origins of cancer: Molecular basis of colorectal cancer. N Engl J Med 2009;361:2449-60.

239. O'Garra A, Chang R, Go N, et al. Ly-1 B (B-1) cells are the main source of B cell-derived interleukin 10. Eur J Immunol 1992;22:711-7. 
240. Kruessel JS, Huang HY, Wen Y, et al. Different pattern of interleukin-1 beta-(IL1 beta), interleukin-1 receptor antagonist- (IL-1ra) and interleukin-1 receptor type I- (IL-1R tI) mRNA-expression in single preimplantation mouse embryos at various developmental stages. J Reprod Immunol 1997;34:103-20.

241. Huang DR, Wang J, Kivisakk P, et al. Absence of monocyte chemoattractant protein 1 in mice leads to decreased local macrophage recruitment and antigenspecific $\mathrm{T}$ helper cell type 1 immune response in experimental autoimmune encephalomyelitis. J Exp Med 2001;193:713-26.

242. Team RDC. R: A Language and Environment for Statistical Computing. 2010.

243. Brasil P. DiagnosisMed: Diagnostic test accuracy evaluation for medical professionals. 2010.

244. Vinterbo SA. gcl: Compute a fuzzy rules or tree classifier from data. 2007.

245. DeLong ER, DeLong DM, Clarke-Pearson DL. Comparing the Areas under Two or More Correlated Receiver Operating Characteristic Curves: A Nonparametric Approach. Biometrics 1988;44:837-845.

246. Hanley JA, McNeil BJ. A method of comparing the areas under receiver operating characteristic curves derived from the same cases. Radiology 1983;148:839-43.

247. Symonds DA, Vickery AL. Mucinous carcinoma of the colon and rectum. Cancer 1976;37:1891-1900.

248. Kakar S, Aksoy S, Burgart LJ, et al. Mucinous carcinoma of the colon: correlation of loss of mismatch repair enzymes with clinicopathologic features and survival. Mod Pathol 2004;17:696-700. 
249. Leopoldo S, Lorena B, Cinzia A, et al. Two subtypes of mucinous adenocarcinoma of the colorectum: clinicopathological and genetic features. Ann Surg Oncol 2008;15:1429-39.

250. Halvorsen TB, Seim E. Influence of mucinous components on survival in colorectal adenocarcinomas: a multivariate analysis. J Clin Pathol 1988;41:106872.

251. Wyatt MG, Houghton PW, Mortensen NJ, et al. The malignant potential of colorectal Crohn's disease. Ann R Coll Surg Engl 1987;69:196-8.

252. Levin KE, Dozois RR. Epidemiology of large bowel cancer. World J Surg $1991 ; 15: 562-7$.

253. Rubio CA, Befrits R. Colorectal adenocarcinoma in Crohn's disease: a retrospective histologic study. Dis Colon Rectum 1997;40:1072-8.

254. Winkler R, Wittmer A, Heusermann U. [Cancer and Crohn's disease]. Z Gastroenterol 2002;40:569-76.

255. Hamilton SR. Colorectal carcinoma in patients with Crohn's disease. Gastroenterology 1985;89:398-407.

256. Choi PM, Zelig MP. Similarity of colorectal cancer in Crohn's disease and ulcerative colitis: implications for carcinogenesis and prevention. Gut $1994 ; 35: 950-4$.

257. Weedon DD, Shorter RG, Ilstrup DM, et al. Crohn's disease and cancer. N Engl J Med 1973;289:1099-103.

258. Ky A, Sohn N, Weinstein MA, et al. Carcinoma arising in anorectal fistulas of Crohn's disease. Dis Colon Rectum 1998;41:992-6. 
259. Moore-Maxwell CA, Robboy SJ. Mucinous adenocarcinoma arising in rectovaginal fistulas associated with Crohn's disease. Gynecol Oncol 2004;93:266-8.

260. Ying LT, Hurlbut DJ, Depew WT, et al. Primary adenocarcinoma in an enterocutaneous fistula associated with Crohn's disease. Can J Gastroenterol $1998 ; 12: 265-9$.

261. Kudo K, Funayama Y, Fukushima K, et al. [Carcinoma arising from ileorectal fistula in a patient with Crohn's disease]. Nippon Shokakibyo Gakkai Zasshi 2007;104:1492-7.

262. Smith R, Hicks D, Tomljanovich PI, et al. Adenocarcinoma arising from chronic perianal Crohn's disease: case report and review of the literature. Am Surg 2008;74:59-61.

263. Kuehle CA, Langhorst J, Ladd SC, et al. Magnetic resonance colonography without bowel cleansing: a prospective cross sectional study in a screening population. Gut 2007;56:1079-85.

264. Alexander RJ, Raicht RF. Purification of total RNA from human stool samples. Dig Dis Sci 1998;43:2652-8.

265. Hasegawa Y, Takeda S, Ichii S, et al. Detection of K-ras mutations in DNAs isolated from feces of patients with colorectal tumors by mutant-allele-specific amplification (MASA). Oncogene 1995;10:1441-5.

266. Il'yasova D, Colbert LH, Harris TB, et al. Circulating levels of inflammatory markers and cancer risk in the health aging and body composition cohort. Cancer Epidemiol Biomarkers Prev 2005;14:2413-8. 
267. Erreni M, Bianchi P, Laghi L, et al. Expression of chemokines and chemokine receptors in human colon cancer. Methods Enzymol 2009;460:105-21.

268. Hojo S, Koizumi K, Tsuneyama K, et al. High-level expression of chemokine CXCL16 by tumor cells correlates with a good prognosis and increased tumorinfiltrating lymphocytes in colorectal cancer. Cancer Res 2007;67:4725-31.

269. Bailey C, Negus R, Morris A, et al. Chemokine expression is associated with the accumulation of tumour associated macrophages (TAMs) and progression in human colorectal cancer. Clin Exp Metastasis 2007;24:121-30.

270. Lewis CE, Pollard JW. Distinct role of macrophages in different tumor microenvironments. Cancer Res 2006;66:605-12.

271. Baier PK, Eggstein S, Wolff-Vorbeck G, et al. Chemokines in human colorectal carcinoma. Anticancer Res 2005;25:3581-4.

272. Dinarello CA. Biologic basis for interleukin-1 in disease. Blood 1996;87:2095147.

273. Locati M, Deuschle U, Massardi ML, et al. Analysis of the gene expression profile activated by the CC chemokine ligand 5/RANTES and by lipopolysaccharide in human monocytes. J Immunol 2002;168:3557-62.

274. Liu W, Reinmuth N, Stoeltzing O, et al. Cyclooxygenase-2 is up-regulated by interleukin-1 beta in human colorectal cancer cells via multiple signaling pathways. Cancer Res 2003;63:3632-6.

275. Sano H, Kawahito Y, Wilder RL, et al. Expression of cyclooxygenase-1 and -2 in human colorectal cancer. Cancer Res 1995;55:3785-9. 
276. Maihofner C, Charalambous MP, Bhambra U, et al. Expression of cyclooxygenase-2 parallels expression of interleukin-1beta, interleukin-6 and NFkappaB in human colorectal cancer. Carcinogenesis 2003;24:665-71.

277. Tsujii M, DuBois RN. Alterations in cellular adhesion and apoptosis in epithelial cells overexpressing prostaglandin endoperoxide synthase 2. Cell 1995;83:493501.

278. Tsujii M, Kawano S, Tsuji S, et al. Cyclooxygenase regulates angiogenesis induced by colon cancer cells. Cell 1998;93:705-16.

279. Kanaoka S, Yoshida K, Miura N, et al. Potential usefulness of detecting cyclooxygenase 2 messenger RNA in feces for colorectal cancer screening. Gastroenterology 2004;127:422-7.

280. Takai T, Kanaoka S, Yoshida K, et al. Fecal cyclooxygenase 2 plus matrix metalloproteinase $7 \mathrm{mRNA}$ assays as a marker for colorectal cancer screening. Cancer Epidemiol Biomarkers Prev 2009;18:1888-93.

281. Lahm H, Petral-Malec D, Yilmaz-Ceyhan A, et al. Growth stimulation of a human colorectal carcinoma cell line by interleukin- 1 and -6 and antagonistic effects of transforming growth factor beta 1. Eur J Cancer 1992;28A:1894-9.

282. Enss ML, Cornberg M, Wagner S, et al. Proinflammatory cytokines trigger MUC gene expression and mucin release in the intestinal cancer cell line LS180. Inflamm Res 2000;49:162-9.

283. Jarry A, Vallette G, Branka JE, et al. Direct secretory effect of interleukin-1 via type I receptors in human colonic mucous epithelial cells (HT29-C1.16E). Gut 1996;38:240-2. 
284. Plaisancie P, Barcelo A, Moro F, et al. Effects of neurotransmitters, gut hormones, and inflammatory mediators on mucus discharge in rat colon. Am J Physiol 1998;275:G1073-84.

285. Van Lieshout EM, Van Doesburg W, Van der Meer R. Real-time PCR of host DNA in feces to study differential exfoliation of colonocytes between rats and humans. Scand J Gastroenterol 2004;39:852-7.

286. Shattuck-Brandt RL, Varilek GW, Radhika A, et al. Cyclooxygenase 2 expression is increased in the stroma of colon carcinomas from IL-10(-/-) mice. Gastroenterology 2000;118:337-45.

287. Sohn KJ, Shah SA, Reid S, et al. Molecular genetics of ulcerative colitisassociated colon cancer in the interleukin 2- and beta(2)-microglobulin-deficient mouse. Cancer Res 2001;61:6912-7.

288. Rudolph U, Finegold MJ, Rich SS, et al. Ulcerative colitis and adenocarcinoma of the colon in G alpha i2-deficient mice. Nat Genet 1995;10:143-50.

289. Berg DE, Kohn MA, Farley TA, et al. Multi-state outbreaks of acute gastroenteritis traced to fecal-contaminated oysters harvested in Louisiana. J Infect Dis 2000;181 Suppl 2:S381-6.

290. Schaub SA, Oshiro RK. Public health concerns about caliciviruses as waterborne contaminants. J Infect Dis 2000;181 Suppl 2:S374-80.

291. Fankhauser RL, Noel JS, Monroe SS, et al. Molecular epidemiology of "Norwalklike viruses" in outbreaks of gastroenteritis in the United States. J Infect Dis 1998;178:1571-8. 
292. Wobus CE, Thackray LB, Virgin HWt. Murine norovirus: a model system to study norovirus biology and pathogenesis. J Virol 2006;80:5104-12.

293. Hsu CC, Wobus CE, Steffen EK, et al. Development of a microsphere-based serologic multiplexed fluorescent immunoassay and a reverse transcriptase PCR assay to detect murine norovirus 1 infection in mice. Clin Diagn Lab Immunol 2005;12:1145-51.

294. Wobus CE, Karst SM, Thackray LB, et al. Replication of Norovirus in cell culture reveals a tropism for dendritic cells and macrophages. PLoS Biol 2004;2:e432.

295. Lindesmith L, Moe C, Lependu J, et al. Cellular and humoral immunity following Snow Mountain virus challenge. J Virol 2005;79:2900-9.

296. Tacket CO, Sztein MB, Losonsky GA, et al. Humoral, mucosal, and cellular immune responses to oral Norwalk virus-like particles in volunteers. Clin Immunol 2003;108:241-7.

297. Qualls JE, Kaplan AM, van Rooijen N, et al. Suppression of experimental colitis by intestinal mononuclear phagocytes. J Leukoc Biol 2006;80:802-15.

298. Qualls JE, Tuna H, Kaplan AM, et al. Suppression of experimental colitis in mice by CD11c+ dendritic cells. Inflamm Bowel Dis 2009;15:236-47.

299. Alex P, Zachos NC, Nguyen T, et al. Distinct cytokine patterns identified from multiplex profiles of murine DSS and TNBS-induced colitis. Inflamm Bowel Dis 2009;15:341-52.

300. Mills CD, Kincaid K, Alt JM, et al. M-1/M-2 macrophages and the Th1/Th2 paradigm. J Immunol 2000;164:6166-73. 
301. Watanabe H, Numata K, Ito T, et al. Innate immune response in Th1- and Th2dominant mouse strains. Shock 2004;22:460-6.

302. Mumphrey SM, Changotra H, Moore TN, et al. Murine norovirus 1 infection is associated with histopathological changes in immunocompetent hosts, but clinical disease is prevented by STAT1-dependent interferon responses. J Virol 2007;81:3251-63.

303. Karst SM, Wobus CE, Lay M, et al. STAT1-dependent innate immunity to a Norwalk-like virus. Science 2003;299:1575-8.

304. Perdue KA, Green KY, Copeland M, et al. Naturally occurring murine norovirus infection in a large research institution. J Am Assoc Lab Anim Sci 2007;46:39-45.

305. Ward JM, Wobus CE, Thackray LB, et al. Pathology of immunodeficient mice with naturally occurring murine norovirus infection. Toxicol Pathol 2006;34:70815.

306. Ekbom A, Daszak P, Kraaz W, et al. Crohn's disease after in-utero measles virus exposure. Lancet 1996;348:515-7.

307. Robertson DJ, Sandler RS. Measles virus and Crohn's disease: a critical appraisal of the current literature. Inflamm Bowel Dis 2001;7:51-7.

308. Lavy A, Broide E, Reif S, et al. Measles is more prevalent in Crohn's disease patients. A multicentre Israeli study. Dig Liver Dis 2001;33:472-6.

309. Pardi DS, Tremaine WJ, Sandborn WJ, et al. Early measles virus infection is associated with the development of inflammatory bowel disease. Am J Gastroenterol 2000;95:1480-5. 
310. Chase K, Seamons A, Treuting P, et al. Murine norovirus, an intercurrent variable in a mouse model of bacterial-induced inflammatory bowel disease. J Am Assoc Lab Anim Sci 2007;46:83.

311. Niidome T, Huang L. Gene therapy progress and prospects: nonviral vectors. Gene Ther 2002;9:1647-52.

312. Katayama K, Wada K, Nakajima A, et al. A novel PPAR gamma gene therapy to control inflammation associated with inflammatory bowel disease in a murine model. Gastroenterology 2003;124:1315-24.

313. Gaboriau-Routhiau V, Rakotobe S, Lecuyer E, et al. The key role of segmented filamentous bacteria in the coordinated maturation of gut helper $\mathrm{T}$ cell responses. Immunity 2009;31:677-89.

314. Ivanov, II, Atarashi K, Manel N, et al. Induction of intestinal Th17 cells by segmented filamentous bacteria. Cell 2009;139:485-98.

315. Wang Z, Gerstein M, Snyder M. RNA-Seq: a revolutionary tool for transcriptomics. Nat Rev Genet 2009;10:57-63. 


\section{Vita}

Aaron Conrad Ericsson was born in Des Moines, Iowa on July 14, 1971. After graduating from Theodore Roosevelt Senior High School in 1989, he began undergraduate studies at the University of Iowa and received his Bachelor of Arts in English in 1994. He eventually founded his own small business, Pundzak/Ericsson Painting, and remained there for several years. As the rigors of owning and operating a small business became increasingly burdensome, Aaron accepted an employment opportunity at the local animal shelter, the Animal Rescue League (ARL). Thus began a long and tortuous journey working with animals. While his work at the ARL was both trying and rewarding, his time there was cut short due to a cat attack resulting a week of hospitalization (seriously). Aaron soon thereafter moved to St. Louis, Missouri and began working as a veterinary technician at Webster Groves Animal Hospital (WGAH). It was here, under the tutelage of Drs. Patrick Tate, John Pletz, and Todd Thomas, that he developed an interest in medicine and science, and his duties quickly shifted from cleaning kennels to assisting in surgery and performing in-house bloodwork. It was also here that Aaron met his wife Jeannie, a uniquely tolerant woman. Aaron soon decided to pursue a career in Veterinary Medicine and enrolled for night classes at the University of Missouri - St. Louis, completing the science courses necessary for application to the University of Missouri, College of Veterinary Medicine. He was accepted to the CVM and began his studies in 2002, simultaneously accepting a position in the Clinical Pathology Laboratory at the Veterinary Medical Teaching Hospital as an after-hours and emergency technician. He remained there for two years, and also worked one year in the lab of Dr. James Schadt, one year as a teaching assistant in veterinary anatomy, and two 
years as a necropsy technician at the Research Animal Diagnostic Laboratory (RADIL). It was through the RADIL and, in particular, Dr. Craig Franklin, that Aaron was once again lured by the siren's song of biomedical research and decided to pursue yet more formal education, in the form of a PhD program in Pathobiology combined with a residency in Comparative Medicine. Upon obtaining his DVM magna cum laude, he began his studies in the MU Comparative Medicine Program, and finished his residency duties in 2009. His research there has focused on the role of the innate immune system in mouse models of enteric disease, namely inflammatory bowel disease (IBD) and colitisassociated colorectal cancer. In particular, his $\mathrm{PhD}$ studies describe his efforts to identify: 1) the contribution of a subset of intestinal dendritic cells expressing the surface marker CD8 $\alpha$ in a mouse model of IBD, and 2) novel methods of noninvasively detecting CAC in a mouse model. Future plans include continuing these studies and also examining the interplay of the innate immune system and intestinal microbiota, hopefully as a faculty member in a university setting. Jeannie and Aaron are now also the proud parents of Joseph Lawrence Ericsson, eventual master of the universe. 